Copyright

by

Martin Ivanov Panevsky

2003 
The Dissertation Committee for Martin Ivanov Panevsky

certifies that this is the approved version of the following dissertation:

\section{Characterization of the Resonant Electromagnetic Mode in Helicon Discharges}

Committee:

Roger Bengtson, Supervisor

Herbert Berk

Mel Oakes

Ken Gentle

Edward Powers

Boris Breizman 
Characterization of the Resonant Electromagnetic Mode in Helicon Discharges

\author{
by \\ Martin Ivanov Panevsky, B.S.
}

\author{
DISSERTATION \\ Presented to the Faculty of the Graduate School of \\ The University of Texas at Austin \\ in Partial Fulfillment \\ of the Requirements \\ for the Degree of \\ DOCTOR OF PHILOSOPHY
}

THE UNIVERSITY OF TEXAS AT AUSTIN

December 2003 
Dedicated to my wife Jane, to my parents Margarita and Ivan, and to my best friends Jazz and Faith. 


\section{Acknowledgments}

I am deeply indebted to my supervisor Dr.Roger Bengtson who envisioned and directed my work on this project. His careful and patient guidance has taught me to look at the big picture in every physics problem and ask the right questions at every stage of my research. I am also grateful for the opportunity to interact with Dr.Boris Breizman and Dr.Alexey Arefiev and benefit and learn from their theoretical mastery. I also thank Dr.Mel Oakes for lending insight into the subtleties of rf circuit analysis. Many thanks to Keith Carter who, I believe, can build and repair anything that an inept graduate student can throw at him. My personal gratitude also goes to Hernan Quevedo for his friendship and help during our months and years of sharing the laboratory. Many people from FRC and IFS have given me support and guidance and I thank them all. I also deeply appreciate the assistance of Dr.John Davis from ECE during the calibration phase of the experiment. Many thanks to SEMATECH for providing the rf sensors we used in our diagnostics. This project would not have been possible without the financial support of ASPL at the Johnson Space Center and especially without the commitment of Dr.Franklin Chang-Diaz. Last but not least, my deepest gratitude goes to my wife Jane, my mother Margarita, and my father Ivan who taught me to look to the stars. 


\title{
Characterization of the Resonant Electromagnetic Mode in Helicon Discharges
}

\author{
Publication No.
}

Martin Ivanov Panevsky, Ph.D.

The University of Texas at Austin, 2003

\section{Supervisor: Roger Bengtson}

This dissertation is motivated by a collaboration between the University of Texas at Austin and NASA on the VASIMR project. The Variable Specific Impulse Magnetoplasma Rocket (VASIMR) is an effort to create a plasmabased thruster. The proposed thruster uses a helicon plasma discharge as its plasma source. An ICRH heating mechanism is then employed to impart energy into the plasma. A magnetic nozzle exhausts the plasma converting the ion gyromotion into superalfvenic longitudinal motion, thus creating thrust.

The physics behind the helicon plasma source is an area of active experimental and theoretical research and we are presenting experimental results which cast the problem of helicon plasma in a new light. Our experimental results point to the existence of a resonant electromagnetic mode in the helicon plasma. The data suggests that this mode is universal in the helicon discharge and can be excited over a wide range of frequencies. The resonant frequency 
is slightly lower than the driving frequency in steady-state. The radial component of the perturbed magnetic field of the resonance mode is peaked at a radius $r^{*}$ where the radial density gradient of the plasma has a maximum. The energy damping rate of the resonant mode is relatively low and cannot deposit the mode energy into the plasma in a single pass. The experimental data also shows that the resonant electromagnetic wave is responsible for depositing most of the available rf energy into the plasma. This supports the central conclusion of a new theory created by B. Breizman and A. Arefiev which predicts that the plasma density gradient forms a potential well which traps the electromagnetic energy. In broader terms, the trapping of the wave in a cavity formed by the density gradients of the plasma both radially and axially provides an explanation to the central dilemma of helicon plasmas: the seeming contradiction between the measured low damping rate of the wave and the measured short damping length. The potential well which traps the wave energy effectively provides an unlimited length scale to the mode allowing it to bounce within the boundaries of the cavity while continuously depositing energy into the plasma. The non-Maxwellian electron population detected in the plasma is linked to the high ionization efficiency of the discharge and can be explained with the operation of the resonant helicon mode.

An experimental method used as an active frequency probe into the plasma impedance is developed and presented. 


\section{Table of Contents}

Acknowledgments $\quad$ v

Abstract vi

List of Tables $\quad \mathrm{x}$

List of Figures $\quad$ xi

$\begin{array}{llr}\text { Chapter 1. Introduction } & 1\end{array}$

Chapter 2. Current Challenges in Understanding Helicon Discharges 9

$\begin{array}{llr}\text { Chapter 3. Experimental Setup } & 16\end{array}$

3.1 Overview of the Helicon Device . . . . . . . . . . . . 16

3.2 RF Matching Network . . . . . . . . . . . . . . . . . . . 19

3.3 RF noise suppression . . . . . . . . . . . . . . . 23

$\begin{array}{ll}\text { Chapter 4. Diagnostics } & 27\end{array}$

4.1 Plasma Impedance Diagnostics and Method . . . . . . . . . . 27

4.2 RF Compensated Langmuir Probe . . . . . . . . . . . . . . . 32

4.3 Magnetic Probe . . . . . . . . . . . . . . . . . . . . . . 39

Chapter 5. Experimental results $\quad 42$

5.1 Langmuir probe results . . . . . . . . . . . . . . . . 43

5.2 Magnetic probe data . . . . . . . . . . . . . . . . . . . . . . . . . . . . . . . . 45

5.3 Plasma impedance data . . . . . . . . . . . . . . . . . 49 
Chapter 6. Analysis 53

6.1 Plasma resonance . . . . . . . . . . . . . . . . . 53

6.2 Theoretical summary . . . . . . . . . . . . . . . . 57

6.3 Physical mechanism of the helicon discharge . . . . . . . . . 58

6.4 Wave equation and magnetic profiles . . . . . . . . . . . 61

6.5 Power flow analysis . . . . . . . . . . . . . . . . 65

6.6 Energy cost per electron-ion pair created . . . . . . . . . . 66

6.7 Stability criterion for a steady-state resonance mode . . . . . . 69

$\begin{array}{lll}\text { Chapter 7. Non-Maxwellian electrons } & 73\end{array}$

7.1 Experimental data . . . . . . . . . . . . . . . 73

7.2 Physical mechanism . . . . . . . . . . . . . . . . . . 80

7.3 A practical approach . . . . . . . . . . . . . 88

$\begin{array}{llr}\text { Chapter 8. Summary } & 91\end{array}$

$\begin{array}{lr}\text { Bibliography } & 94\end{array}$

$\begin{array}{lr}\text { Vita } & 98\end{array}$ 


\section{List of Tables}

5.1 Two sets of parameters for the helicon discharge under investigation. . . . . . . . . . . . . . . 43

6.1 Resonance parameters. . . . . . . . . . . . . . 56

6.2 Power loss channels in the discharge. . . . . . . . . . . . . . 69

7.1 Characteristics of the population of non-Maxwellian electrons in the high-density discharge. . . . . . . . . . . . 79 


\section{List of Figures}

1.1 Basic configuration of the Variable Specific Impulse Magnetoplasma Rocket. . . . . . . . . . . . . . . . . . 3

1.2 The specific impulse can be modulated, allowing for trajectory and mission optimization. (Diagram courtesy of NASA.) . . .

3.1 Schematic diagram of the helicon machine, the power delivery system, magnetic coils, and diagnostic probes and sensors. . .

3.2 An early phase in the development of the helicon plasma source at UT Austin. Shown in the foreground is the matching network and some of the boxes shielding the rf sensors. Also visible is the Pyrex tube, the helicon antenna, and the water-cooled magnetic coils producing the dc magnetic field. The glow inside the Pyrex tube is the helicon discharge. . . . . . . . . . .

3.3 L-type matching network used for an inductive load. The parameters of the capacitors shown pertain to our helicon experiment. . . . . . . . . . . . . . . . . .

3.4 Plasma resistance versus antenna current at zero reflected power and fixed $r f$ power. . . . . . . . . . . . . .

3.5 A significant rf noise suppression is achieved by placing a grounded Faraday shield around the matching network and antenna. A grounded Faraday shield is also placed around the primary rf generator. . . . . . . . . . . . . .

3.6 A rf choke for reducing the high-frequency common mode noise. 26

4.1 Thevenin equivalent circuit. . . . . . . . . . . . . . . 28

4.2 Real component of the equivalent output impedance as seen at the antenna terminals.

4.3 Imaginary component of the equivalent output impedance as seen at the antenna terminals. . . . . . . . . . . .

4.4 Imaginary component of the antenna impedance as a function of frequency. . . . . . . . . . . . . . .

4.5 RF compensated Langmuir probe head. The probe is perpendicular to $B_{0}$ and the floating electrode is oriented along $B_{0}$. . 
4.6 Blocking inductors and bypass capacitor inside the Langmuir probe head. . . . . . . . . . . . . . . .

4.7 The Langmuir probe is shown as it sits inside the vacuum chamber. The probe head is perpendicular to the axis of the machine and to the dc magnetic field. The floating electrode is oriented along the dc magnetic field and is designed to track the changes in the plasma induced by the rf power. . . . . . . . . . . .

4.8 (a) Schematic diagram of a modified B-dot coil, (b) equivalent electrical circuit of a modified B-dot coil and connection to a $50 \Omega$ load (Loewenhardt, Blackwell, and Zhang, 1993). . . . . .

4.9 Shown is the actual magnetic probe. The probe head is made of boron nitride and the three mutually perpendicular coils are wrapped on it. The signal from each coil is transmitted through a rigid coaxial cable which adds structural strength to the whole probe assembly. . . . . . . . . . . . . .

5.1 Density and temperature measured by the rf compensated Langmuir probe $30 \mathrm{~cm}$ downstream from the center of the antenna.

5.2 Density measured by the Langmuir probe $84 \mathrm{~cm}$ downstream from the center of the antenna. . . . . . . . . . . . .

5.3 Amplitude profiles of the $B_{r}$ component of the electromagnetic wave taken at a fixed axial location within the helicon discharge for the high-density case. The amplitude of $B_{r}$ at the driving frequency is scaled to fit on the plot. . . . . . . . . .

5.4 Profiles of the magnetic field amplitude of the helicon electromagnetic wave taken at a fixed axial location within the helicon discharge for the low-density case. . . . . . . . . . . . .

5.5 Axial dependance of the total wave magnetic field amplitude in the high-density helicon discharge. . . . . . . . . . . . .

5.6 Wave magnetic field amplitude in the helicon chamber. The axial direction is measured from the edge of the antenna. . . .

5.7 Imaginary component of the plasma impedance showing a resonance response close to the driving frequency. . . . . . . . .

6.1 Equivalent circuit for the plasma resonance model. . . . . . .

6.2 Curve fit of the imaginary component of the plasma impedance plotted together with the experimental data being fitted. . . .

6.3 Real component of the plasma impedance calculated from Eq. 6.7 using the experimental values of the resonance parameters. Also shown is the frequency response of the blocking LC filter. The halfwidth of the filter is less than $1 / 10$ the halfwidth of the resonance curve. . . . . . . . . . . . . . . . . 
6.4 State functions and energy levels in a quantum well. This corresponds to a potential cavity formed by the density gradient in the helicon discharge. The state function $\psi$ is replaced by $E$ in the plasma case. . . . . . . . . . . . . .

6.5 Approximate theoretical profiles of $B_{r}$ predicted by the radially localized resonant theory of helicon discharges. . . . . . . . .

6.6 Exact solutions for the radial component of the wave magnetic field for the $m=+1$ and $m=+2$ modes based on the radially localized helicon mode theory. . . . . . . . . . . . .

6.7 Radial component of the wave magnetic field as predicted by the uniform plasma theory. . . . . . . . . . . . . . .

6.8 Profile of the power absorbed by the plasma as the discharge goes through a resonance. . . . . . . . . . .

6.9 Profile of $T_{e}$ obtained from power balance by treating the plasma density $n$ as a linear parameter. . . . . . . . . . . . .

6.10 The resonance profile of $T_{e}$ is obtained from power balance and the constant profile of $T_{e}$ is obtained from particle balance. The two intersection points are the equilibrium $T_{e}$ of the discharge. Point $\mathrm{A}$ is unstable and point $\mathrm{B}$ is stable. . . . . . . . . . .

7.1 High-density discharge, plasma potential estimated at approximately $55 \mathrm{~V}$, radial position of the probe $r \approx 0.0 \mathrm{~cm} . . .$. .

7.2 High-density discharge, plasma potential estimated at approximately $54 \mathrm{~V}$, radial position of the probe $r \approx 1.0 \mathrm{~cm} . . .$. .

7.3 High-density discharge, plasma potential estimated at approximately $35 \mathrm{~V}$, radial position of the probe $r \approx 2.0 \mathrm{~cm}$. . . . . .

7.4 Relative enhancement of the electron number density at the observed energy for the non-Maxwellian electrons compared to a Maxwellian distribution. . . . . . . . . . . . . . .

7.5 Theoretical estimate of the parallel resonance condition for the high-density discharge. Zero crossing would indicate a parallel resonance. The discharge conditions predict near-resonance conditions at $r \approx 0 \mathrm{~cm}$ and at the plasma periphery, plus a spike at $r \approx 1 \mathrm{~cm} \ldots \ldots \ldots \ldots . \ldots . \ldots$

7.6 Experimental details in the radial profile of the non-Maxwellian electron population for the high-density discharge. The observed spikes in non-Maxwellian electron density are consistent with the theoretical estimate. . . . . . . . . . . . 
7.7 Theoretical estimate of the parallel resonance condition for the low-density discharge. Zero crossing indicates a parallel resonance. The discharge conditions predict near-resonance conditions at $r \approx 0.4 \mathrm{~cm}, r \approx 1.0 \mathrm{~cm}$, and a resonance at the plasma periphery.

7.8 Experimental details in the radial profile of the non-Maxwellian electron population for the low-density discharge. The observed spikes in non-Maxwellian electron density are consistent with the theoretical estimate. . . . . . . . . . . . . .

7.9 Electron energy estimate based on the parallel resonance mechanism over one rf period. . . . . . . . . . . . . . . .

7.10 Second derivative portion of the $\mathrm{I}-\mathrm{V}$ trace for three different input voltages on the capacitive antenna at $R \approx 0 \mathrm{~cm}$. The graph zooms on the non-Maxwellian portion of the I-V trace. .

7.11 Second derivative portion of the I-V trace for three different input voltages on the capacitive antenna at $R \approx 2.0 \mathrm{~cm}$. The graph zooms on the non-Maxwellian portion of the I-V trace. . 


\section{Chapter 1}

\section{Introduction}

This work is part of an ongoing collaboration between a research group at the University of Texas at Austin headed by Dr.Roger Bengtson and Dr.Boris Breizman and the Advanced Space Propulsion Laboratory at NASA Johnson Space Center under the leadership of Dr. Franklin Chang-Diaz. The project goal is to create a plasma-based space propulsion system capable of carrying humans to Mars and back safely. The research group at UT Austin is tasked with understanding the physics behind the proposed thruster.

The plasma propulsion system would have to be fast, reliable, and fuel efficient in order to minimize the fuel weight, and capable of variable thrust in order to allow optimization of the trajectory. The most restrictive requirement is improving the fuel efficiency in order to reduce the enormous fuel weight that a conventional chemical rocket would be carrying. Reducing the fuel consumption while maintaining reasonable thrust means that the device must have a very high specific impulse. The specific impulse is the amount of thrust per unit weight of fuel spent:

$$
I_{s p}=\frac{T}{(\mathrm{~d} W / \mathrm{d} t)}=\frac{v_{e}(\mathrm{~d} m / \mathrm{d} t)}{g(\mathrm{~d} m / \mathrm{d} t)}=\frac{v_{e}}{g}
$$


where $T$ is the thrust produced by the engine, $W$ is the weight of the fuel burnt, $v_{e}$ is the exhaust velocity, and $g$ is the acceleration due to gravity. In order to have a high specific impulse and low fuel consumption, the exhaust velocity has to be maximized. In a conventional rocket engine, the exhaust velocity is determined by the energy stored in the chemical bonds of the fuel. That energy is typically a fraction of an electronvolt. On the other hand, based on the requirements for a mission to Mars, a realistic propulsion system would require exhaust velocities in the order of 100's eV. At that energy the fuel will be ionized and thus the propulsion system will necessarily be plasma-based.

The Variable Specific Impulse Magnetoplasma Rocket (VASIMR) is one of NASA's research projects to design, build, and test a prototype for a plasma thruster [10]. Fig. 1.1 shows the basic components of VASIMR. Light gas, hydrogen or helium, is injected into the vacuum chamber. A helicon antenna powered by a rf source ionizes the gas which streams downstream confined by the dc magnetic field. The type of plasma discharge chosen to be the plasma source is the helicon discharge. Its main advantage is the ability to produce high plasma density with a moderate energy input. In fact, the rf energy expended to create the cold helicon plasma is a small fraction of the total energy required for the operation of the device. The plasma density in the helicon discharge has been demonstrated experimentally to be proportional to the dc magnetic field, therefore high intensity magnetic fields will be employed on the envisioned prototype. The field is produced by superconducting magnets spaced in such a way as to create a uniform axial magnetic field, especially 


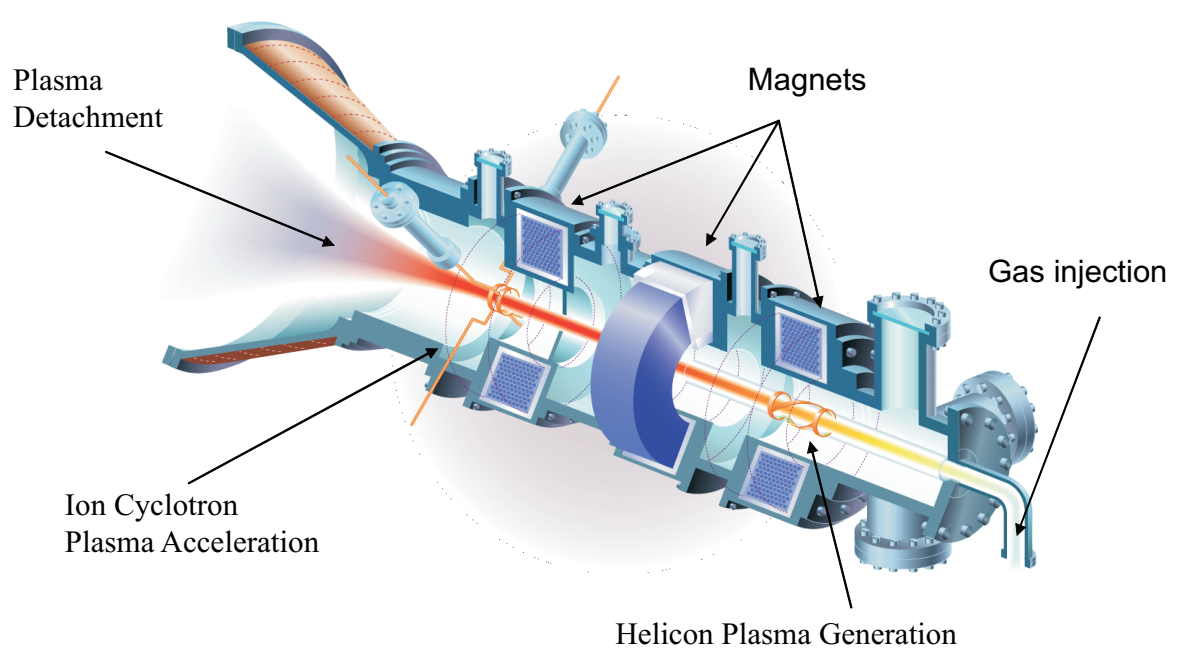

Figure 1.1: Basic configuration of the Variable Specific Impulse Magnetoplasma Rocket.

in the helicon portion of the machine. From the helicon chamber the plasma enters the ion-resonance heating chamber where a second rf antenna creates a circularly polarized electric field with frequency matching the ion cyclotron frequency. The ICRH chamber is where conditions for resonant power absorption are created and the largest percentage of total power is deposited into the plasma imparting 100's eV per ion. At this stage the ion energy is mostly the energy of their gyromotion. The plasma then enters the magnetic nozzle section where the high intensity dc magnetic field diverges and decreases. Since the ions preserve their magnetic moment $\mu=\frac{m_{i} v_{\perp}^{2}}{2 B_{z}}$ and their total kinetic en$\operatorname{ergy} \mathcal{E}=m_{i} v_{\perp}^{2} / 2+m_{i} v_{\|}^{2} / 2$, as the ions enter the region of decreasing magnetic field their rotational energy is transferred to longitudinal motion. The ions 
leaving the system with energies of 100's of electronvolts provide thrust to the device in the opposite direction. The magnetic nozzle region also provides another vital function: detachment of the plasma from the dc magnetic field. This happens when the energy density of the plasma is much bigger than the energy density of the magnetic field and the diverging magnetic field is simply a small perturbation to the plasma flow. The energy density of the magnetic field scales as $1 / A^{2}$ as required by magnetic flux conservation, where $A$ is the cross section of the magnetic nozzle. On the other hand, the energy density of the plasma flow scales as $1 / A$, as required by ion flux conservation. The energy density of the plasma quickly becomes larger than the energy density of the magnetic field and the plasma breaks free.

The high exhaust velocity translates into a high specific impulse of about 20,000 for the plasma rocket, compared to a conventional chemical rocket. By comparison, the space shuttle has a specific impulse of about 250 . Another advantage of the plasma thruster is the ability to modulate the specific impulse by controlling the parameters of the discharge. As Fig. 1.2 shows, the plasma engine can be configured for a low-thrust, low-fuel consumption mission, or for a high-thrust, high-fuel consumption mission. Overall, the fuel consumption is modest, on the order of grams of fuel per second. This high fuel efficiency comes at the expense of high energy consumption. In order to impart 100's eV of energy per plasma particle, VASIMR will require a compact but high energy density source. Currently, the only viable candidate for an energy source with these specifications is a nuclear reactor. 


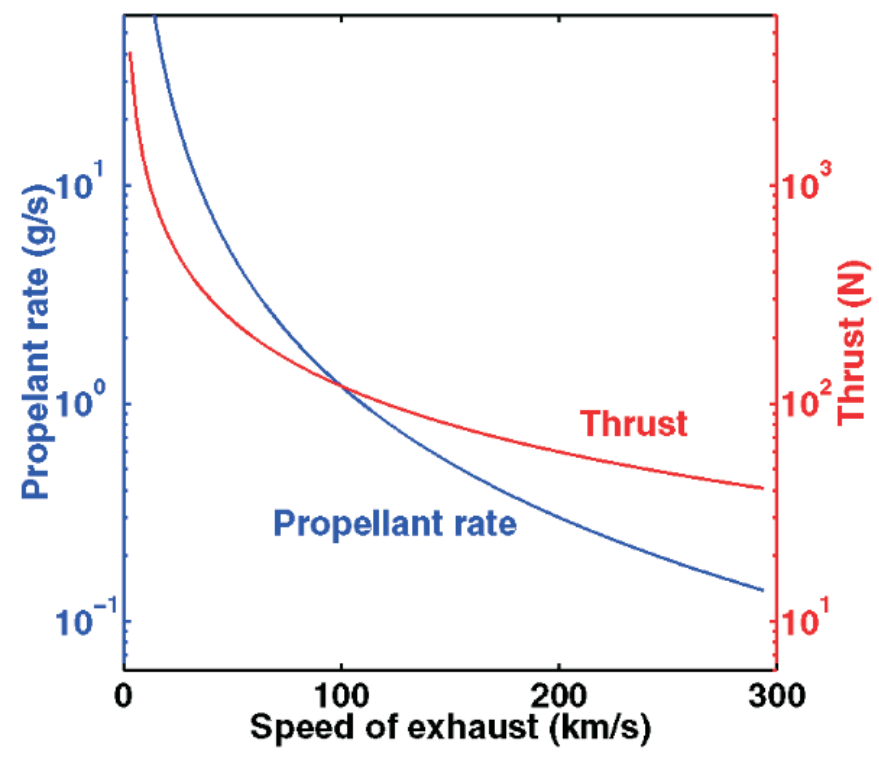

Figure 1.2: The specific impulse can be modulated, allowing for trajectory and mission optimization. (Diagram courtesy of NASA.)

The VASIMR plasma thruster has additional advantages as well:

- Low fuel consumption allows for a continuous operation of the engine throughout the flight.

- Electrodeless design with magnetic insulation

- Capability for powered mission aborts

There are many theoretical and engineering questions that need to be answered before VASIMR can become a reality. Efficient operation of the ICRH heating 
requires at a minimum a high density plasma source. That is why the helicon discharge was chosen. Helicons carry the promise of efficient operation but present a significant challenge in terms of explaining the underlying physics. Helicon discharges are under active theoretical and experimental study not only for plasma-based propulsion but for plasma processing as well. The fundamental challenge posed by the helicon discharge is the fact that experimentally they exhibit a fast power absorption rate which results in an intense, compact discharge with a short damping length. On the other hand, it is not clear theoretically what is the physical mechanism responsible for these high power absorption rates. When I joined the VASIMR team at the University of Texas at Austin in the fall of 1998, the task at hand was to develop a theory and create an experiment that can shed light on the helicon "mystery". Ultimately, the goal is to understand the physics behind the helicon discharge which will allow optimization of the plasma source. I focused my research on the experimental side of the problem. Our first attempt was to adapt an existing plasma device for the helicon experiment. We soon discovered that the helicon discharge has a number of unique requirements and we decided to build a dedicated helicon machine starting from scratch. After the machine was completed, work began on the diagnostics. That posed its own unique set of challenges largely due to the intense rf environment of the discharge. The development of the diagnostics went hand in hand with the development of a set of software tools for data acquisition. The framework of our experiment started to take shape after we had extensive discussions with Dr. Boris Breiz- 
man and Dr. Alexey Arefiev who had recently developed a theory of radially localized helicon modes in nonuniform plasma [9]. Even at that early stage it seemed that this theory, if operable, can provide answers to some of the questions posed by the helicon discharge.

We believe that the experimental results and analysis presented in this thesis are an important step in extending our understanding of helicon plasma. From a scientific point of view, our results offer answers to some of the perplexing questions about helicons. From a practical point of view, we suggest a concrete way of exploiting our results for the purpose of optimizing the discharge.

This thesis consists of eight chapters which include the Introduction (Chapter 1) and the Summary (Chapter 8).

Chapter 2 presents the framework and method of our experiment in light of the many unanswered questions about helicon discharges.

Chapter 3 presents the experimental setup and the helicon device that we have constructed.

Chapter 4 discusses the diagnostics and the unique set of challenges that the helicon discharge presents to constructing proper plasma probes. We also focus on the issues associated with collecting data in the noise-filled helicon environment.

Chapter 5 presents the experimental results as they pertain to the radially localized helicon modes.

In Chapter 6 we analyze our data and present the physics behind it. 
We analyze the existence of the radially localized mode, its characteristics, physical mechanism, power balance, and significance in sustaining the helicon discharge.

In Chapter 7 we present experimental data showing the existence of fast non-Maxwellian electrons in the discharge. We also show that the theory of radially localized helicon modes can be used to provide an elegant explanation for the existence of these electrons in the discharge. We also suggest a possible way of improving the efficiency of the plasma source based on our data and conclusions.

Some of the key results of this thesis have been submitted for publication to Physics of Plasmas. A preliminary version of these results was presented at the 44th Annual Meeting of the Division of Plasma Physics of the American Physical Society [28]. 


\section{Chapter 2}

\section{Current Challenges in Understanding Helicon Discharges}

Helicon plasma discharges are subject to active theoretical and experimental work $[2,13,14,18,30]$ because of their ability to produce high density plasmas under easily accessible laboratory conditions: modest input power and a relatively low magnetic field. The aim of our experiment is to understand the physics behind a proposed plasma thruster that uses a helicon discharge as its plasma source. We received further motivation after the publication of a theoretical work on helicon plasmas by B. Breizman and A. Arefiev [9] and extensive discussions with the authors. Unlike an earlier theory of helicon plasma which assumes a uniform density profile, this approach incorporates the radial density gradient in the discharge in a natural way and predicts a dispersion relation which has the observed $\omega \propto k_{\|}^{2}$ dependance. This theory also predicts important features of the helicon discharge which we have confirmed in our experiment. We believe that the experimental results presented here in conjunction with that theory constitute an important new step in the research and understanding of helicon plasmas. We present experimental results which allow us to treat the helicon plasma as a harmonic oscillator driven by the $\mathrm{rf}$ power. This oscillator operates in a state of near resonance with a resonant 
frequency slightly lower than the driving frequency as is required for a steady state discharge, suggesting that the helicon plasma operates under conditions of resonant power absorption. The damping rate of this oscillator is relatively low which means that in a single-pass system the helicon wave would need many wavelengths in order to deposit its energy into the plasma. This creates a seeming contradiction between the fast power absorption which helicon plasmas are known for, and a calculated damping length which is usually longer than the size of the helicon machine. The theory of Breizman and Arefiev [9] predicts that the radial density gradient in the helicon plasma creates a potential well which traps the helicon wave and we present experimental evidence showing weak damping along with strong power absorption. Our results also show a clear axial density gradient in the plasma. The theory can be extended to include this effect which suggests that the helicon wave is also trapped in the axial direction [8]. Therefore, the helicon plasma forms a resonant cavity which confines the electromagnetic wave. The electromagnetic energy launched by the antenna bounces back and forth in the potential well of the plasma as many times as is required by the damping rate until it deposits its energy. Our power balance analysis shows that this trapped electromagnetic mode is the dominant electromagnetic mode in the helicon plasma responsible for at least $80 \%$ of the power absorbed by the discharge.

Despite an impressive body of publications on the subject, both theoretical and experimental, a clear understanding of the the underlying physics of helicon plasma is still lacking. The current theoretical framework of the heli- 
con discharge is centered on several seminal papers published by Boswell [5-7] and Chen et al $[2,13,14]$. There are, however, several significant discrepancies between the theoretical predictions and the experimental observations. While the theory was formulated for a uniform plasma, the experimental facts show that a steep radial density gradient is a hallmark of all helicon discharges. The standard helicon dispersion relation is

$$
k k_{\|}=\frac{e \mu_{0} n_{0} \omega}{B_{0}} \quad \text { where } \quad k=\sqrt{k_{\perp}^{2}+k_{\|}^{2}}
$$

In cylindrical helicon discharges, the antenna usually sets $k_{\|}$while $k_{\perp} \approx 2 \pi / a$, where $a$ is approximately the plasma radius. Using the parameters of our experiment as a typical example, the length of our half-wavelength antenna is $L_{\text {antenna }}=0.14 \mathrm{~m}, \lambda_{\|}=0.28 \mathrm{~m}$ which sets $k_{\|}=21 \mathrm{~m}^{-1}$. The plasma radius is $a \approx 0.03 \mathrm{~m}$ and $k_{\perp} \approx 2.1 \times 10^{2} \mathrm{~m}^{-1}$, the plasma density $n_{0}=1.2 \times 10^{18} \mathrm{~m}^{-3}$, and the static magnetic field $B_{0}=0.0627 \mathrm{~T}(627 G)$. A substitution into Eq. (2.1) shows that the helicon dispersion relation requires the driving frequency to be $f=186 \mathrm{MHz}$ which is a factor of 14 higher than the actual driving frequency of $13.56 \mathrm{MHz}$. This is a typical result when applying Eq. (2.1) and a significant discrepancy which is reported in various helicon studies. To resolve this, some authors $[17,21]$ have arbitrarily postulated that $k_{\perp}=0$ in order to obtain the experimentally observed dependance $\omega \propto k_{\|}^{2}$. Another difficulty arises when we use Eq. (2.1) to derive the axial decay length for helicon mode damping. We can write $k_{\|}=\beta_{\|}-\mathrm{i} \alpha_{\|}$, where $\alpha_{\|}^{-1}$ is the axial decay length, and $\omega \rightarrow \omega-\mathrm{i} \gamma$ in order to introduce damping, where $\gamma$ is energy damping 
rate. Substituting this into Eq. (2.1) we obtain

$$
\alpha_{\|}^{-1}=\frac{\omega_{c e}}{\gamma k_{\perp}} \quad \text { when } \quad k_{\perp} \gg k_{\|}
$$

and

$$
\alpha_{\|}^{-1}=\frac{2 \omega_{c e}}{\gamma k_{\|}} \quad \text { when } \quad k_{\perp} \ll k_{\|}
$$

Again we use the parameters of our experiment which will be presented in more detail later, $\omega_{c e} \approx 1 \times 10^{10} \mathrm{~s}^{-1}$ and the experimentally determined damping rate $\gamma=2.4 \times 10^{6}$. Substituting into Eq. (2.2) we obtain $\alpha_{\|}^{-1} \approx 13 \mathrm{~m}$ which is more than an order of magnitude higher than the size of the discharge in our experiment as well as in numerous other helicon experiments where the power absorption is highly localized and centered on the helicon antenna.

In an effort to account for the wave absorption, Chen has suggested that Landau damping plays an important role in the helicon discharge [13]. The expression for the Landau damping rate has been verified experimentally [24, $35]$ and is given in [13] by

$$
\nu_{L D}=2 \sqrt{\pi}\left(c^{2} / \omega_{p e}^{2}\right) k_{\perp}^{2} \xi^{3} \exp ^{-\xi^{2}}
$$

where $\xi=\omega /\left(k_{\|} \sqrt{2} v_{t h}\right)$ and $v_{t h}=\sqrt{\left(e T_{e} / m\right)}$. Using the parameters of our experiment gives $v_{t h}=8.4 \times 10^{5} \mathrm{~m} \mathrm{~s}^{-1}, \xi \approx 3.4$ and $\nu_{L D} \approx 1.4 \times 10^{-3} \mathrm{~s}^{-1}$. This result and other theoretical and experimental work by Chen and others have shown $[11,30]$ that Landau damping plays negligible (if any) role in the power absorption mechanism of helicon discharges. 
Another explanation for the experimentally observed efficiency of power deposition in helicons is the excitation of a short-wavelength, nearly electrostatic Trivelpiece-Gould wave in the helicon discharge which serves as an energy sink. There are two proposed channels for that mechanism: a direct excitation channel of the TG wave by the antenna and a mode conversion channel where the helicon wave excites the Trivelpiece-Gould wave. This occurs in the plasma region where conditions exist to satisfy the dispersion relation of both waves. The dispersion relation of the TG wave is given by [3]

$$
\frac{k_{\|}^{2}}{k_{\perp}^{2}}=\frac{\omega^{2}\left(\omega_{p e}^{2}+\omega_{c e}^{2}-\omega^{2}\right)}{\left(\omega_{c e}^{2}-\omega^{2}\right)\left(\omega_{p e}^{2}-\omega^{2}\right)}
$$

The approximations applicable to our experiment are $\omega_{p e}^{2} \gg \omega_{c e}^{2}, \omega^{2}$ and $k_{\perp} \gg$ $k_{\|}$and Eq. 2.5 simplifies to

$$
\omega=\omega_{c e} \frac{k_{\|}}{k}
$$

The direct channel of exciting the electrostatic wave has been investigated [30] and in that respect a convenient way to write the dispersion relation of the Trivelpiece-Gould wave excited by an antenna of radius $a$ is given by

$$
B=\left(m_{e} c / e\right) a_{m p} \omega / k_{\|} a
$$

where $a_{m p}$ is the $p^{t h}$ root of the $m^{\text {th }}$ order Bessel function $J_{m}\left(a_{m p}\right)=0$. The discrete integer $m$ is the azimuthal number of the wavefields $E, B \sim$ $\exp \mathrm{i}(m \theta+\vec{k} \cdot \vec{r}-\omega t)$ and usually $m=0, \pm 1$. Currently it is believed that $[3$, 30] the TG waves are not efficiently excited directly by helicon antennas and have a negligible contribution to the power balance at $B_{0}>100 G$. The halfwavelength, right-helical antenna that we are using excites predominantly a 
$m=+1$ mode. The largest amplitude of the Trivelpiece-Gould wave will occur for $a_{11}=3.83$ and substituting into Eq. (2.7) the parameters of our experiment and solving for $B_{0}$, we obtain $B_{0} \approx 88 \mathrm{G}$. For most helicon plasma devices including ours, such low magnetic fields are not applicable since it is known experimentally that the plasma density increases with $B_{0}$ and high plasma density is the usual operational regime of helicon discharges.

The mode conversion channel is currently under investigation and it is believed that in a high- $B_{0}$ helicon discharge the mode conversion takes place on the outer plasma surface only [29]. In fact, the radial density gradient present in all helicon discharges is a suppressing factor in this case. Numerical results confirm that the contribution of the mode conversion channel to the overall power absorption is not high.

Clearly, the questions of what the physical mechanism of a helicon discharge is, and what provides their high rate of energy absorption are still unanswered.

In this experiment we treat the plasma as a forced harmonic oscillator with damping. The force driving the oscillator is the main $1 \mathrm{~kW}$ rf frequency of $13.56 \mathrm{MHz}$. We explore the frequency response of the plasma by looking at the plasma impedance, especially in the frequency space around the driving 13.56 MHz. In particular, we want to explore the possibility that there exists a helicon resonance mode close to the main driving frequency. If such a mode exists, its resonance frequency, damping rate, and magnetic structure are of considerable importance, as well as the percentage of the total power absorbed by that mode. We investigate this by superimposing a second, low-power vari- 
able frequency on the discharge using the same antenna and detecting the plasma response to the variable frequency. The main rf power creates and sustains the discharge and sets its parameters. The variable frequency signal can be thought of as an active frequency probe into the plasma. It is low power and allows us to treat the problem linearly by interpreting the variable frequency effects as a perturbation which does not change the conditions of the discharge. An array of diagnostics is employed to collect the relevant data. 


\section{Chapter 3}

\section{Experimental Setup}

\subsection{Overview of the Helicon Device}

The helicon source for this experiment is shown in Fig. 3.1. Both the main rf generator and the variable frequency generator couple to the antenna through a L-type matching network [22]. To prevent power flow from the main source to the variable frequency source a high-Q parallel LC resonant circuit was constucted and tuned to $13.56 \mathrm{MHz}$. The impedance at resonance for such a circuit is given by $Z=(2 \pi f L)^{2} / R$. As a result less than $2 \mathrm{~W}$ of power leaks from the main power source to the variable frequency generator. The halfwidth of the filter is less than $1 / 10$ of the halfwidth of the plasma impedance response. The filter is also carefully calibrated as is explained in Chapter 4 . We use a type III antenna with a $180^{\circ}$ helical twist instead of the horizontal legs of the Nagoya antenna. The antenna length is $L_{\text {antenna }}=14 \mathrm{~cm}$. The antenna launches a wave with the wave vector $\overrightarrow{k_{\|}}$pointing downstream from the antenna where $k_{\|}=\pi m / L_{\text {antenna }}$ and $\lambda_{\|}=2 \pi / k_{\|}$. The antenna can excite $m=+1$ or $m=-1$ mode based on the direction of the dc magnetic field. In the current configuration, the antenna launches predominantly the $m=+1$ mode. The mode number $m$ indicates the helicity of the helicon electric field. For $m>0$ the direction of rotation of the helicon electric field coincides with 


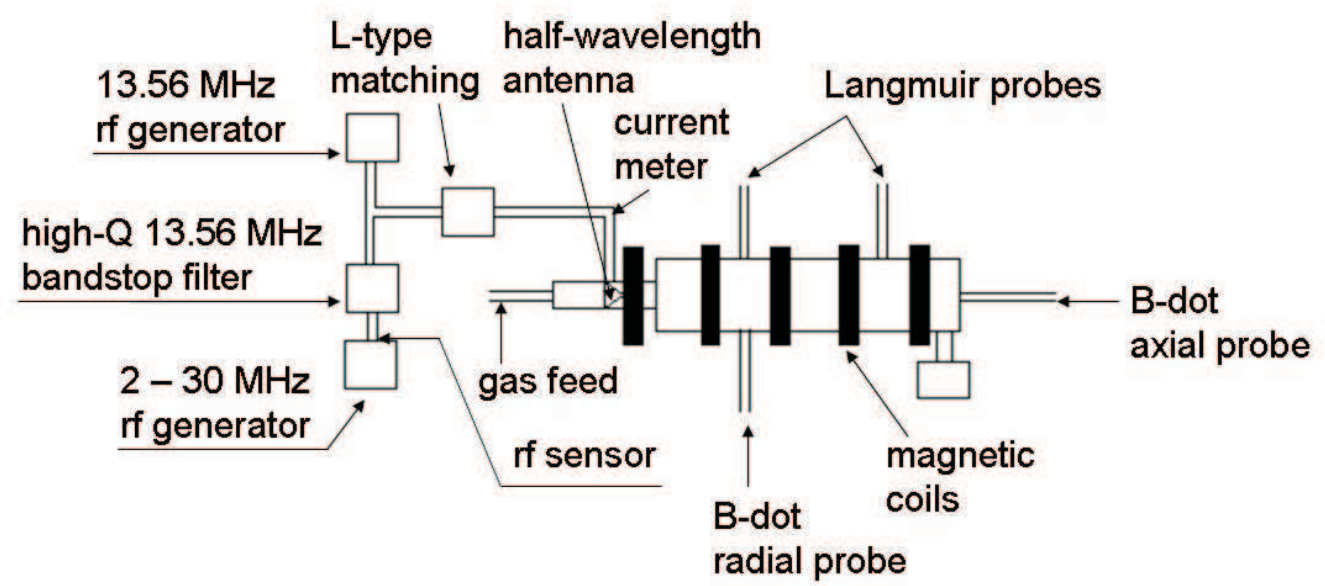

Figure 3.1: Schematic diagram of the helicon machine, the power delivery system, magnetic coils, and diagnostic probes and sensors.

the direction of ion gyromotion. For $m<0$ the direction of wave rotation coincides with the direction of electron gyromotion. The antenna couples to the plasma through a Pyrex glass tube $30 \mathrm{~cm}$ long and $4 \mathrm{~cm}$ in diameter which is bolted to a cylindrical stainless vacuum chamber with an internal diameter of $9.7 \mathrm{~cm}$ and length of $120 \mathrm{~cm}$. The pyrex tube also provides the gas inlet through which argon gas is fed into the vacuum chamber. Fig. 3.2 shows the helicon machine before a Faraday shield was placed around the antenna and matching network as one of the steps aimed at improving the signal-to-noise ratio in the data. We need to point out that in an effort to reduce the gas flow so that we do not overwhelm the turbo pump, a gate valve was placed between the vacuum chamber and the pump. As a result, the neutral pressure 


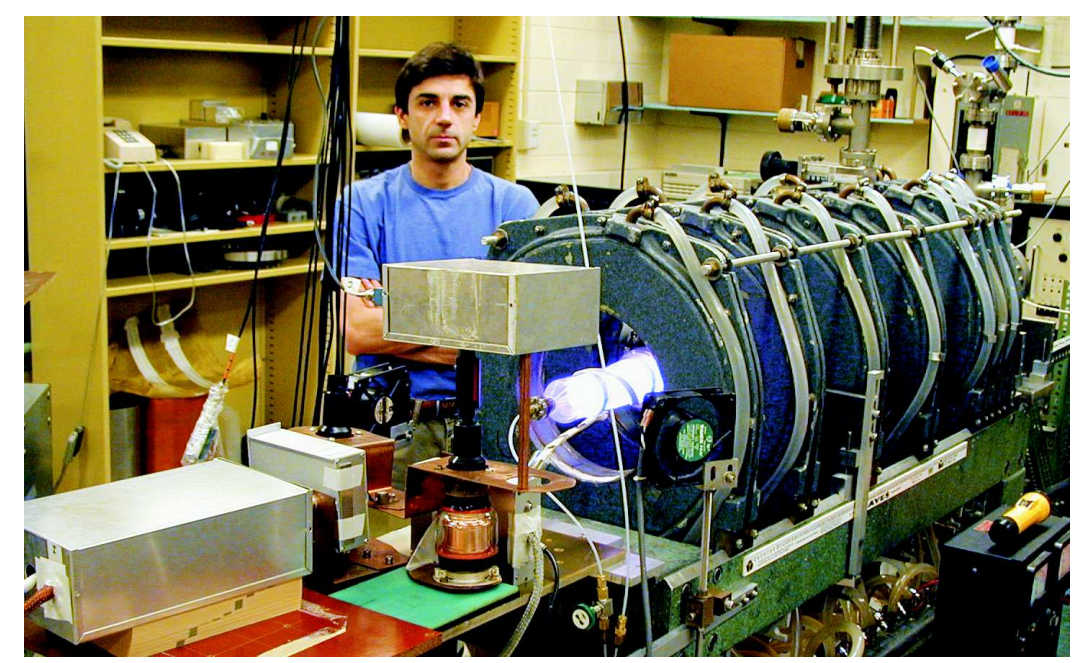

Figure 3.2: An early phase in the development of the helicon plasma source at UT Austin. Shown in the foreground is the matching network and some of the boxes shielding the rf sensors. Also visible is the Pyrex tube, the helicon antenna, and the water-cooled magnetic coils producing the dc magnetic field. The glow inside the Pyrex tube is the helicon discharge.

of several mTorr required for operation is achieved with a minimal gas flow. The vacuum chamber including the Pyrex tube is surrounded by five evenly spaced magnetic coils which provide a dc magnetic field of up to $1.9 \mathrm{kG}$. We operate the main power source in a pulsed regime to allow for cooling of the matching network and antenna, where the duration of a cycle is $5 \mathrm{~s}$ with a $20 \%$ duty cycle. This translates to a plasma pulse of $1 \mathrm{~s}$ every $5 \mathrm{~s}$. The main rf generator provides $1 \mathrm{~kW}$ of power per pulse at $13.56 \mathrm{MHz}$, while the variable frequency generator provides $10-15 \mathrm{~W}$ in the frequency range of $9-15 \mathrm{MHz}$. 


\subsection{RF Matching Network}

The delivery of power from the rf generator to the antenna-plasma load requires an impedance match between them. The condition for a maximum power transfer is given by

$$
Z_{L}=Z_{e q}^{*}
$$

which says that the we maximize the power to the load if the impedance of the load is the complex conjugate of the equivalent output impedance of the source.

In a helicon discharge the antenna and plasma are strongly inductive. The source is a rf generator with the standard $50 \Omega$ output impedance. This creates a severe impedance mismatch which prevents the rf power from being delivered to the load because the power launched by the rf generator is reflected at the load. The result is that the discharge fails to ignite. Moreover, the reflected power has the potential of damaging the rf generator. Most modern rf generators have a fail-safe circuitry which detects excessive reflected power and shuts the generator down. All this necessitates the use of a matching network. This network is placed between the generator and the load. The matching network combined with the load effectively creates a new load $Z_{L}^{\text {effective }}$. A properly tuned matching network has an impedance such that

$$
Z_{L}^{\text {effective }}=Z_{e q}^{*}
$$

The equivalent output impedance of the generator has a $50 \Omega$ real component and no imaginary component. Therefore, we need a matching network that 


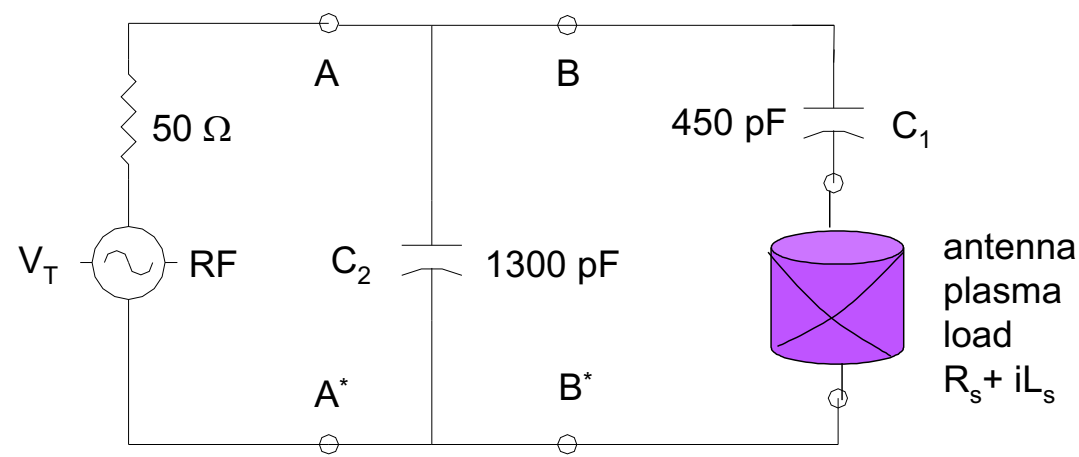

Figure 3.3: L-type matching network used for an inductive load. The parameters of the capacitors shown pertain to our helicon experiment.

will have $\operatorname{Re}\left[Z_{L}^{\text {effective }}\right]=50 \Omega$ and $\operatorname{Im}\left[Z_{L}^{\text {effective }}\right]=0$. Since the plasma and antenna are inductive, it is natural to use a capacitive matching network to cancel the inductive component of the load. The matching network we are using is known as a L-type network. It is shown on Fig. 3.3 There are various ways to calculate the required values for $C_{1}$ and $C_{2}$ once the values of $R_{T}$ and $Z_{s}=R_{s}+\mathrm{i} L_{s}$ are known. Since the circuit contains several components in parallel, the easiest procedure is to use admittance instead of impedance as is presented in [22]. The admittance looking to the right of terminals B-B* is

$$
Y_{A} \equiv G_{A}+\mathrm{i} B_{A}=\frac{1}{R_{s}+\mathrm{i}\left(X_{1}+X_{s}\right)}
$$

where the conductance is

$$
G_{A}=\frac{R_{s}}{R_{s}^{2}+\left(X_{1}+X_{s}\right)^{2}}
$$


and the susceptance is

$$
B_{A}=-\frac{X_{1}+X_{s}}{R_{S}^{2}+\left(X_{1}+X_{s}\right)^{2}}
$$

and $X_{1}=-\left(\omega C_{1}\right)^{-1}, X_{s}=\omega L_{s}$. For maximum power transfer we want $G_{A}=1 / R_{T}=1 / 50$ which we solve to obtain $X_{1}$ and thus find $C_{1}$. Substitute into Eq. 3.3c to obtain $B_{A}$. We choose $C_{2}$ by noting that the susceptance of $C_{2}$ has to cancel the susceptance $B_{A} \Rightarrow \omega C_{2}=-B_{A}$ and this gives us $C_{2}$. In reality, it is impossible to know in advance the exact impedance of the plasmaantenna load, hence the capacitors $C_{1}$ and $C_{2}$ are variable capacitors that can be fine-tuned. The values of the capacitors that give us a complete match are given in Fig. 3.3. In our experiment, the main $\mathrm{rf}$ generator delivers $1 \mathrm{~kW}$ of power to the load and the reflected power is less than $1 \%$.

There is one last point about fine-tuning the matching network: just because the matching network is tuned in a way that minimizes the reflected power does not necessarily mean that the system is optimized. The minimized reflected power does not tell us if the current through the antenna is maximized which is the only way to ensure that we are delivering maximum power to the antenna and plasma. If the current through the antenna is not maximized, the matching network is operating in a regime where there is a large current circulating between the rf generator, terminals $\mathrm{A}-\mathrm{A}^{*}$ and capacitor $C_{2}$. As a result, instead of delivering maximum power to the load some of the power is being discharged through the components of the matching network itself. To optimize the operation of the network, we place a current meter in the loop formed by capacitor $C_{2}$, terminals B-B*, and the load. Then we fine-tune the 


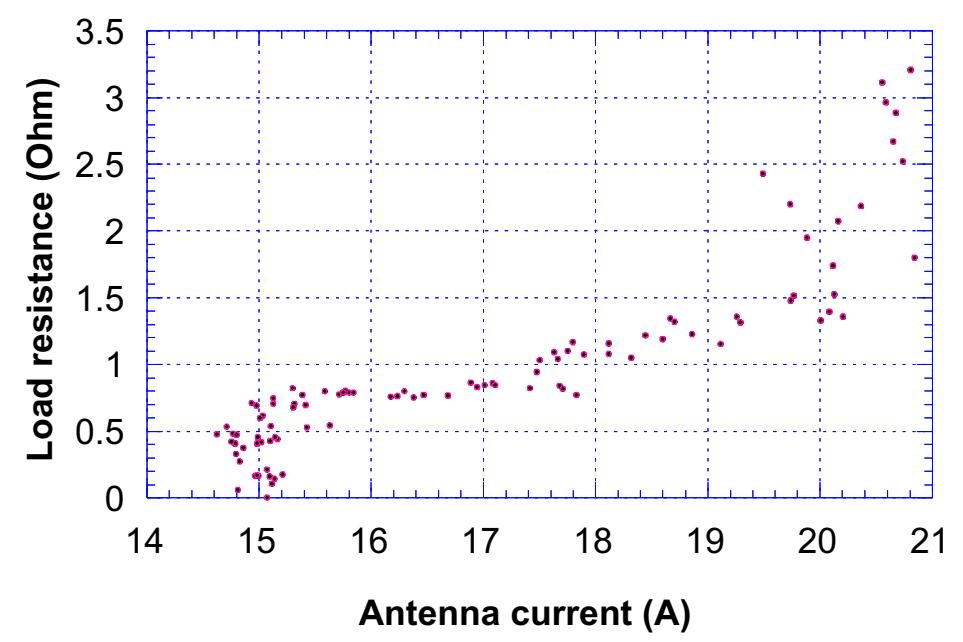

Figure 3.4: Plasma resistance versus antenna current at zero reflected power and fixed rf power.

capacitors to maximize the antenna current while maintaining a minimum on the total reflected power. Fig. 3.4 shows that the benefits from maximizing the antenna current are quite significant. These results are obtained using a rf sensor placed in the matching network to track the plasma response to changes in the antenna current. Our data shows:

- A $5 \%$ decrease in the antenna current can lead to a $57 \%$ drop in coupling resulting in a strong nonlinear decrease in power delivery to the plasma.

- The Q of the effect is approximately 20 . 


\subsection{RF noise suppression}

The helicon discharge creates a noise-filled environment which if unchecked results in an unfavorable signal-to-noise ratio, impeding data collection. The rf noise comes from the primary rf generator at $13.56 \mathrm{MHz}$, from the antenna and matching network, from the plasma, and from the vacuum vessel which is grounded and thus saturates the ground with rf noise. There is no "magic bullet" for reducing this pervasive rf noise. The solution is to implement a host of measures which in their totality will bring the noise level to a minimum. There are two areas that we need to focus on in order to reduce the rf noise: suppressing the noise at the source and further suppressing and separating the noise from the data signal at the data collection point. In a helicon discharge experiment these two areas are necessarily separated. A modern data collection and analysis equipment is highly computerized. The computers used for data acquisition are adversely affected by the strong magnetic field associated with the helicon discharge and by the fluctuations of the $\mathrm{AC}$ ground which affects the computer power supply. This necessitates a separation of about 25 - $30 \mathrm{ft}$ between the helicon device and the data acquisition electronics. Reducing the rf noise at the source requires the simultaneous implementation of several steps:

- Eliminate all ground loops. This requires careful planning of the equipment layout and interconnections. The machine ground is usually the main grounding point. As a last resort, some of the additional equipment connected to the machine can be floated. 


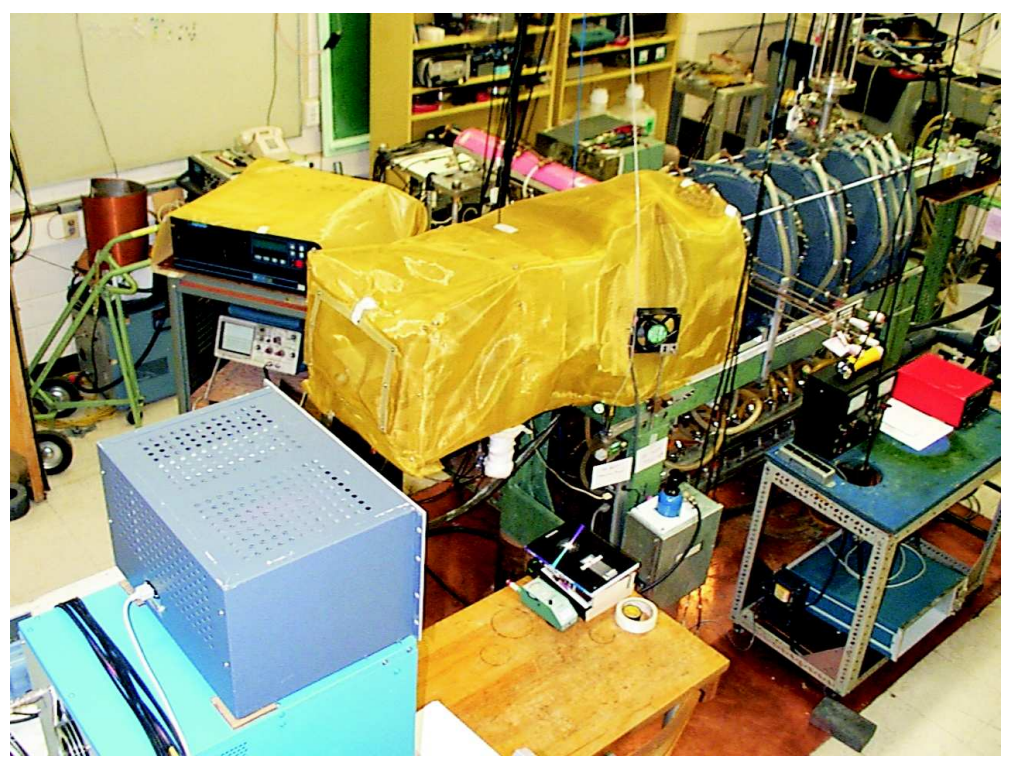

Figure 3.5: A significant rf noise suppression is achieved by placing a grounded Faraday shield around the matching network and antenna. A grounded Faraday shield is also placed around the primary rf generator.

- Grounded Faraday shields are useful if placed around the parts of the machine which are the main noise source: the rf generators, matching network, and antenna. Additionally, Faraday shields in the form of grounded metallic boxes are useful in preventing the rf noise from reaching sensitive measuring equipment like rf sensors, digital filters, analog-to-digital converters and their power supplies.

- If the data-carrying signal is of frequency which is sufficiently different from the main driving frequency, use filters at the source. If the data signal needs to be amplified, place the amplifier as close as possible to the point where the data signal is created, not at the point where 
data is collected. In this way the amplifier will boost the data signal only, and not amplify the noise signal which will accumulate on the data transmission line between the machine and the data collection point.

- Use Triax coaxial cables instead of the standard coaxial cable to carry the data signal. The outer conductor of the Triax cable must be grounded on the machine end only.

- Use isolation amplifiers between the plasma machine and the data acquisition system. This helps eliminate potential ground loops, protects the data acquisition system from voltage spikes emanating from the plasma device, and allows for a separate computer ground.

Minimizing the rf noise and separating it from the data signal at the data acquisition system requires the simultaneous implementation of the following steps:

- Use filters placed immediately before the data acquisition point

- The common mode noise on the outer conductor of a coaxial cable can be eliminated by a rf choke of the kind shown in Fig. 3.6. The rf choke is created by tightly winding a data-carrying coaxial cable around a ferrite core. As explained in [20], this configuration has proved very effective in reducing the common mode noise on the outer conductor without affecting the actual coaxial signal. 


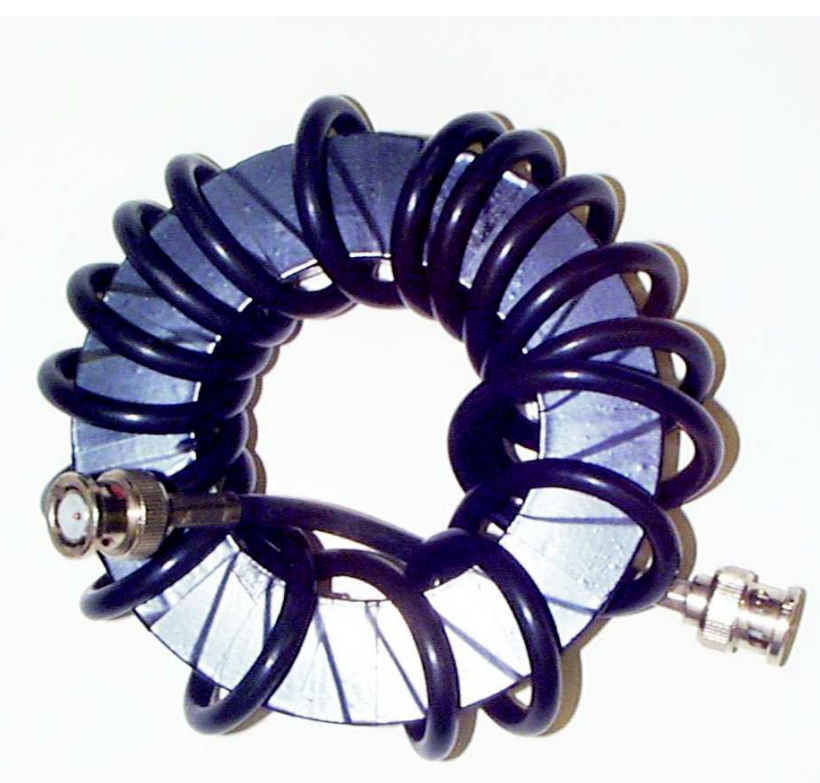

Figure 3.6: A rf choke for reducing the high-frequency common mode noise.

- Configure the data acquisition equipment for a differential input operation. This further helps isolate the common mode noise from the data signal.

- Use spectral analysis to separate the signal coming from the plasma probes from the rf noise.

- When aliasing can be safely ruled out, use the capabilities of the data acquisition cards to further digitally process the data signal.

After implementing all these steps in our experiment we were able to greatly improve our signal-to-noise ratio and begin data collection. 


\section{Chapter 4}

\section{Diagnostics}

\subsection{Plasma Impedance Diagnostics and Method}

The plasma impedance diagnostics consist a rf sensor [27] and a sensitive current meter. The rf sensor is placed between the variable frequency generator and the blocking LC resonator. It is capable of measuring the voltage, current, and phase angle at that location for the entire frequency range of $9-15 \mathrm{MHz}$. The current meter is placed directly on the antenna and accurately measures the antenna current. Information about the plasma is extracted from these sensors using their readings and a database of frequencydependent impedance information for the entire system of generators, matching network, blocking LC resonator, and antenna.

From the standpoint of circuit analysis, the helicon discharge in our experiment consists of a power delivery system and a load. The power delivery system contains the rf generators, LC blocking resonator, matching network, and antenna. The load is the plasma. Precise information about the power delivery system allows us to separate the effects of that system from the response of the plasma. This is especially true if the mathematical formalism used creates a clear and explicit distinction between the load (plasma) and the rest of the system. The basic concept is illustrated in Fig. 4.1. If the power 


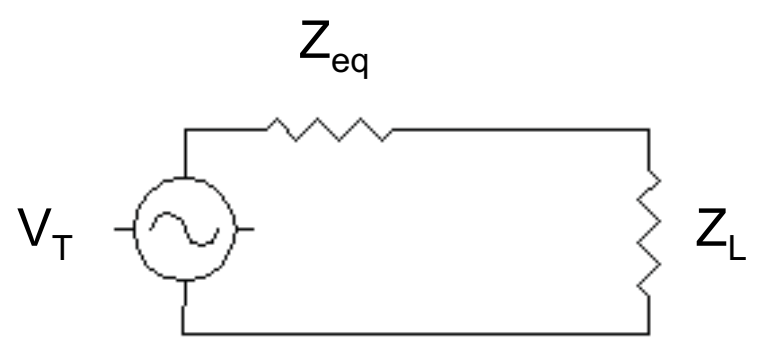

Figure 4.1: Thevenin equivalent circuit.

source is a voltage source (as rf generators are), a circuit of any complexity is reduced to a Thevenin equivalent circuit. The circuit consists of a source voltage, an equivalent output impedance which represents the impedance of the entire power delivery system, and a load impedance. The source voltage $V_{T}$ is defined as the open circuit voltage at the terminals of the load. The output impedance $Z_{e q}$ is defined as the impedance presented to the load with all the sources in the circuit turned off [16]. The source current $I_{N}$ is defined as the current at the load terminals if the load is replaced by a short circuit. The usefulness of these concepts is evident from the expressions which govern their use:

$$
\begin{gathered}
V_{L}=V_{T} \frac{1}{1+Z_{e q} / Z_{L}} \\
I_{L}=\frac{V_{T}}{Z e q} \frac{1}{1+Z_{L} / Z_{e q}}=I_{N} \frac{1}{1+Z_{L} / Z_{e q}}
\end{gathered}
$$

where $V_{L}$ is the voltage on the load, $I_{L}$ is the current through the load, $Z_{e q}$ is the equivalent output impedance, and $Z_{L}$ is the load impedance. In a rf environment all these quantities are complex. It is clear that using this frame- 
work we can ask questions about the plasma load which is the only relevant quantity in the circuit, without having to solve for the entire circuit every time. Ultimately, we want to investigate the plasma impedance in frequency space. Therefore, we have to create a database for $Z_{e q}$ and $I_{L}$ as a function of frequency in order to use Eq. 4.1b which is the more convenient expression in our case. The first step is to create an analytical model of the power delivery system based on the measured impedance values of its components: inductors, capacitors, rf generators, and antenna. The model predicts the current amplitude through the antenna with no plasma and using the variable frequency generator as a voltage source. We keep $V_{T}$ fixed in the model and experiment. We measure the antenna current (see Fig. 3.1) in the frequency range of $9-15 \mathrm{MHz}$ in steps of $50 \mathrm{kHz}$ and compare the results with the current predicted by the model. There is a good agreement between theory and experiment which gives us confidence that we have a good overall understanding of the frequency response of the power delivery system. The next step is to refine our database. To do this we calibrate the rf sensor (see Fig. 3.1) for the frequency range of interest in steps of $50 \mathrm{kHz}$ using a digital network analyzer and replace the helicon antenna by a short circuit. Fig. 6.3 shows the calibrated frequency response of the LC filter. A repeat of the frequency scan then gives the system impedance at every frequency point as calculated by the rf sensor. We substitute this impedance database into the model and calculate the Norton current. The actual measured Norton current amplitude is then used as a feedback into the impedance database. We continue this iteration 


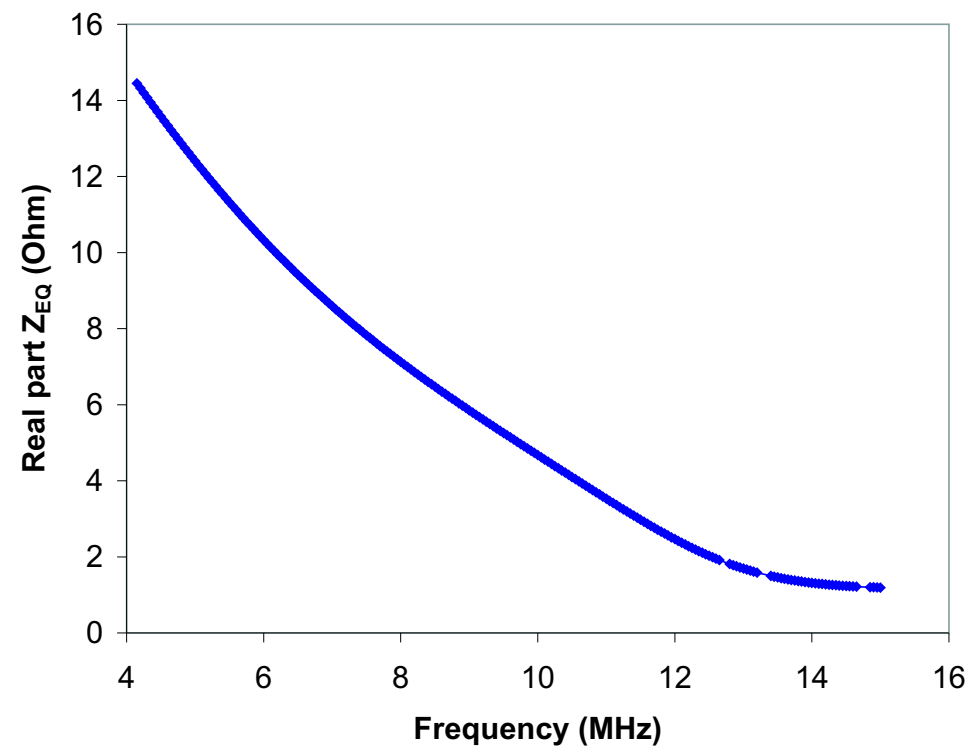

Figure 4.2: Real component of the equivalent output impedance as seen at the antenna terminals.

process several times until we are satisfied with the accuracy of our impedance database which contains the complex values of $I_{N}$ and $Z_{e q}$. Fig. 4.2 and Fig. 4.3 present the calibrated impedance database for the equivalent output impedance as seen by the antenna. This is the impedance $Z_{e q}$ which is used in Eq. 4.1b. We then reconnect the antenna and treat the antenna as a load and use our impedance database and analytical model to accurately calculate the antenna impedance in this frequency range. Again, we use the antenna current amplitude as a feedback in order to iteratively refine the impedance values of the antenna at every frequency point. This entire calibration process is performed with no plasma. Fig. 4.4 shows the calibrated database of the 


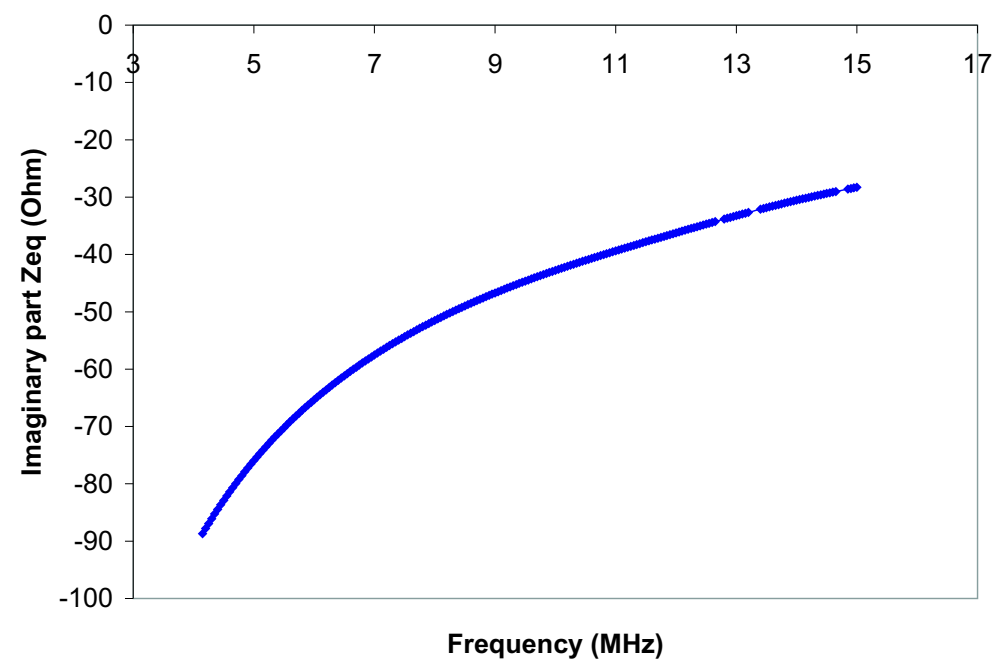

Figure 4.3: Imaginary component of the equivalent output impedance as seen at the antenna terminals.

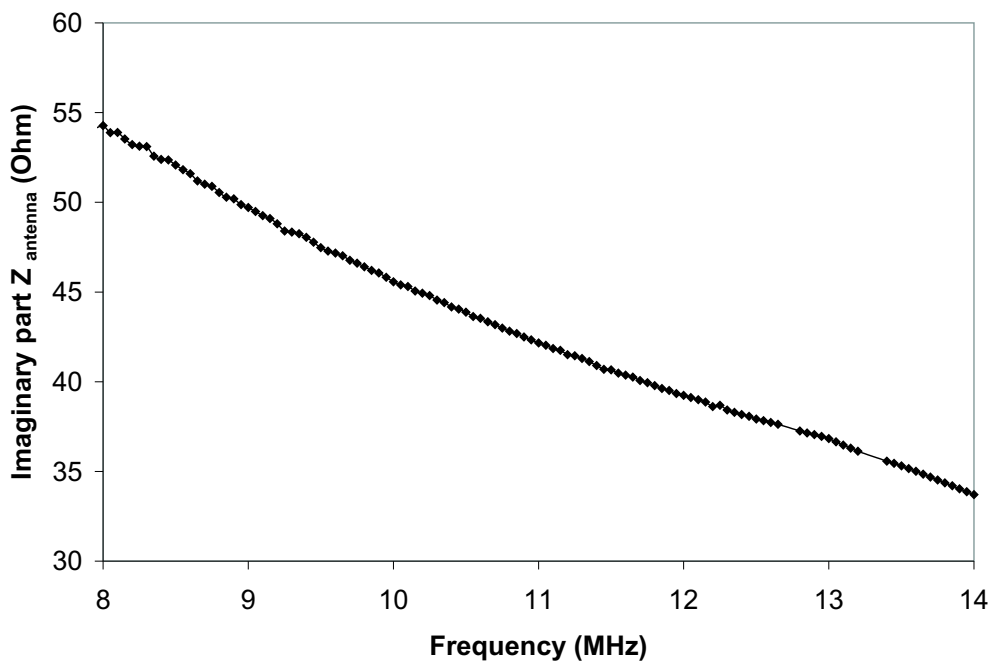

Figure 4.4: Imaginary component of the antenna impedance as a function of frequency. 
antenna impedance as a function of frequency.

Note that the imaginary antenna impedance in the frequency space close to the driving frequency is essentially the complex conjugate of the equivalent output impedance $\operatorname{Im}\left[Z_{e q}\right]$ as required by Eq. 3.1.

Once the calibration is complete, we have two separate diagnostic tools for investigating the plasma impedance:

- The rf sensor which gives us the combined impedance of power delivery system and plasma. Knowing the impedance of the power delivery system allows us to extract the plasma impedance.

- The impedance database for $I_{N}$ and $Z_{e q}$ as functions of frequency and the current meter on the antenna measuring $I_{L}$ allow us to use Eq. $4.1 \mathrm{~b}$ to calculate the plasma impedance at every frequency point.

The frequency resolution of the spectrum analyzer used for data collection is $1 \mathrm{kHz}$ which allows us to distinguish the driving frequency signal from the active frequency probe signal at every point of the frequency scan except at the very frequency point $(13.56 \mathrm{MHz})$ when the two signals completely overlap.

\subsection{RF Compensated Langmuir Probe}

The use of standard Langmuir probe theory in helicon discharges is constrained by three factors: probe size and plasma temperature which determine if collisions in the probe sheath have to be taken into account, a strong dc magnetic field which distorts the $\mathrm{I}-\mathrm{V}$ characteristic of the probe, and $\mathrm{rf}$ 
oscillations of the plasma potential which also strongly affect the probe trace. Fig. 4.5 shows the Langmuir probe. The probe head is boron nitride, the probe tip holders are aluminum oxide, and the probe tips are molybdenum, $1.5 \mathrm{~mm}$ in diameter and $2.5 \mathrm{~mm}$ long which gives a characteristic probe area of $d^{2}=13.5 \mathrm{~mm}^{2}$. The floating electrode is molybdenum as well, $1.5 \mathrm{~mm}$ in diameter and $35.2 \mathrm{~mm}$ long. The tip separation is $3.6 \mathrm{~mm}$. At $T_{e} \approx 6 \mathrm{eV}$, the Debye length $\lambda_{D e} \approx 2 \times 10^{-3} \mathrm{~cm}$ and the thickness of a collisionless plane sheath [15] is given by $s=\lambda_{D e}\left(\frac{e|V|}{T_{e}}\right)^{3 / 4} \approx 2.44 \mathrm{~mm}$ and $s^{2}=5.95 \mathrm{~mm}^{2}$, while from [12] $\lambda_{e}=\frac{3.4 \times 10^{13} T_{e}^{2}}{n \ln \Lambda} \approx 39 \mathrm{~cm}$. Then $\lambda_{e}^{2} \gg d^{2} \gg s^{2}$ and as classified in [15], we are in the collisionless conventional thin sheath regime. The addition of the dc magnetic field reduces the electron current and distorts the probe characteristic in the retardation region of the I-V trace [31]. Only for a large negative voltage does the electron current vary as $\exp (e V / k T)$. In a strong magnetic field the effective probe area for electron flux collection is the probe cross section along the magnetic field lines. The ion collection is usually not severely affected as can be seen from the parallel and perpendicular diffusion coefficients [22] $D_{\perp}=\frac{D_{\|}}{1+\omega_{c}^{2} \tau_{c}^{2}}$, where $\omega_{c}$ is the gyrofrequency and $\tau_{c}$ is the mean collision time. Then $\omega_{c}=1 / \nu_{m}$ where $\nu_{m}$ is the momentum transfer frequency which in our discharge is dominated by the electron-neutral collisions. At $T_{e}=6 \mathrm{eV}, \nu_{m}=n_{g} K_{e l}$ where $n_{g}$ is the neutral gas density and $K_{e l}$ is the elastic collision rate constant $\left(K_{e l} \equiv\langle\sigma v\rangle\right)$. Then for the parameters of our discharge, $\nu_{m}=6 \times 10^{7} \mathrm{~s}^{-1}, \tau=2 \times 10^{-8} \mathrm{~s}$, and $\omega_{c}=1 \times 10^{10} \mathrm{~s}^{-1}$. For electrons $\omega_{c} \tau_{c} \approx 10^{2}$ and the electron radial diffusion is strongly inhibited. For ions, $\omega_{c}$ 
decreases as $m / M, \tau_{c}$ increases as $(M / m)^{1 / 2}$, and $\omega_{c} \tau_{c} \propto(m / M)^{1 / 2}$. Therefore, the ion diffusion is not severely limited and the usual standard expressions for the electron and ion probe currents can be used if the ion gyroradius is large compared to the sheath, and with the appropriate corrections for the effective probe area. Another experimental consideration when sweeping the probe in the presence of a dc magnetic field is that the probe voltages do not extend much beyond the floating potential [33]. The reason is that near the plasma potential cold electrons with small gyroradii are predominant and therefore the saturation electron current is difficult to reach since it is diffusion limited. In our experiment $r_{c i} \gtrsim s$ and we use the following expressions for the I-V trace:

$$
\begin{gathered}
I=I_{e}-I_{i} \quad\left(I_{e}>0, I_{i}<0\right) \\
I_{e}=\frac{1}{4} e A n_{s} \bar{v}_{e} \exp \frac{\left(V_{B}-\Phi_{p}\right)}{T_{e}} \\
I_{i}=e n_{s} u_{B} A
\end{gathered}
$$

where $u_{B}$ is the Bohm velocity, $\bar{v}_{e}=\left(8 e T_{e} / \pi m\right)^{1} / 2$, and $V_{B}-\Phi_{p}<0$ is the potential between the probe and the plasma $\left(V_{B}\right.$ is the probe potential and $\Phi_{p}$ is the plasma potential). The fact that we are in the collisionless thin sheath regime also means that we can use Druyvesteyn's formula to analyze the Langmuir probe data. In this regime the electron distribution function is proportional to $\mathrm{d}^{2} I_{e} / \mathrm{d} V^{2}$. In our experiment it is more convenient to use the electron energy distribution function [22] which is a variation of Druyvesteyn's formula:

$$
g_{e}(V)=\frac{2 m}{e^{2} A}\left(\frac{2 e V}{m}\right)^{1 / 2} \mathrm{~d}^{2} I_{e} / \mathrm{d} V^{2}
$$




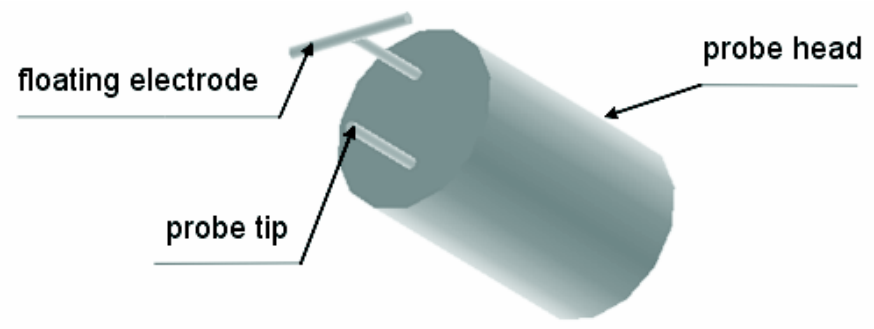

Figure 4.5: RF compensated Langmuir probe head. The probe is perpendicular to $B_{0}$ and the floating electrode is oriented along $B_{0}$.

$$
V=\Phi_{p}-V_{B}>0 \quad \text { (retarding potential region) }
$$

The effect of the rf signal further complicates the analysis of the probe trace. It is known [4] that in rf generated plasmas the I-V trace of the Langmuir probe is distorted by the oscillations that the probe sees in the plasma potential. The plasma floating potential seen by the probe in a rf environment is given by

$$
\frac{V_{F}}{T_{e}}=-\ln \frac{I_{0}(A)}{\alpha} \sqrt{\frac{M}{2 \pi m}}
$$

where $I_{0}(A)$ is the modified Bessel function, $A$ is the amplitude of the rf oscillation, and $\alpha$ is a constant of the order of unity. As $A$ grows, $V_{f}$ becomes more negative and the entire I-V trace is shifted to more negative values. The trace is no longer exponential [4] and the end result is that the probe gives the wrong estimate of $T_{e}$, plasma potential and floating potential. One solution to avoid the large voltage swings in $V_{\text {plasma }}-V_{\text {probe }}$ is to place inductors in the probe head that will present a large impedance to the rf oscillation but 
will let through the low-frequency sweep signal. Chen has shown [32] that the rf-induced changes in the probe sheath capacitance give rise to harmonics of the fundamental rf frequency thus generating harmonics in the probe current, most notably a second harmonic. The inductors in the probe have to block the first and the second harmonic. The condition for rf compensation [32] is given by the following expression

$$
Z_{\text {inductor }} \gg Z_{\text {sheath }}\left(\frac{\left|V_{r f}\right|}{T_{e}}-1\right)
$$

Using the procedure outlined in [32] and the parameters of our discharge we calculate $R_{\text {sheath }} \approx 1.5 \mathrm{k} \Omega, C_{\text {sheath }} \approx 0.5 \mathrm{pF}$, and $\left|Z_{\text {sheath }}\right| \approx 1.5 \mathrm{k} \Omega$. We have measured the amplitude of the rf voltage on the antenna to be $V_{r f} \approx 500 \mathrm{~V}$ which substituted into Eq. 4.5 shows that rf compensation requires $Z_{\text {inductor }} \gg$ $123 \mathrm{k} \Omega$. There are no miniature inductors with such high inductance values. Instead, we can reduce the impedance of $Z_{\text {sheath }}$ in Eq. 4.5 by placing a large floating electrode in parallel with the probe tip as shown in Fig. 4.5. The surface area ratio between the floating electrode and the probe tip gives the factor of decrease of $Z_{\text {sheath }}$. In our case this factor is 14 which means that for the blocking inductors we now require $Z_{\text {inductor }} \gg 9 \mathrm{k} \Omega$. As Fig. 4.6 shows, two inductors of $82 \mu \mathrm{H}$ each block the primary frequency, and two inductors of $47 \mu \mathrm{H}$ block the second harmonic. There are two more pairs of similar inductors placed outside the probe. The total impedance provided by the blocking inductors is $28 \mathrm{k} \Omega$ at $13.56 \mathrm{MHz}$ and $32 \mathrm{k} \Omega$ at the second harmonic. As can be seen in Fig. 4.6 the probe tip connects to the floating electrode through a $2 \mathrm{nF}$ capacitor. The purpose of this capacitor is to provide a low impedance path 


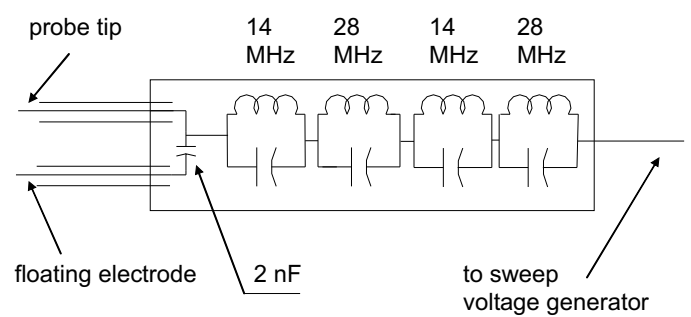

Figure 4.6: Blocking inductors and bypass capacitor inside the Langmuir probe head.

for the rf signal in order to reduce the sheath impedance while blocking the low frequency sweep signal so that the probe can be biased. At $13.56 \mathrm{MHz}$ the $2 \mathrm{nF}$ capacitor provides $6 \Omega$ resistance to the $\mathrm{rf}$ signal and $1.6 \mathrm{M} \Omega$ resistance to the dc bias and probe sweep signal. The reason the floating electrode is along the dc magnetic field is threefold: besides the rf induced oscillations of the plasma potential we expect the plasma to also have a radial potential gradient, while in the axial direction we expect a uniform plasma potential. The second reason is trying to minimize the cross section that the floating electrode presents to the plasma in order to minimize the effect the probe is having on the plasma. The third and most important reason is that in the presence of a dc magnetic field the effective lengths perpendicular to the magnetic filed are reduced by a factor $\eta_{e, i}=\sqrt{1+x_{e, i}^{2}}$ where $x_{e, i}=\lambda_{e, i} / r_{c e, c i}$ and $r_{c e, c i}$ is the gyroradius. Under the conditions of our experiment this reduction is significant and would have reduced the effective area of the floating electrode to the point of making it ineffective. 


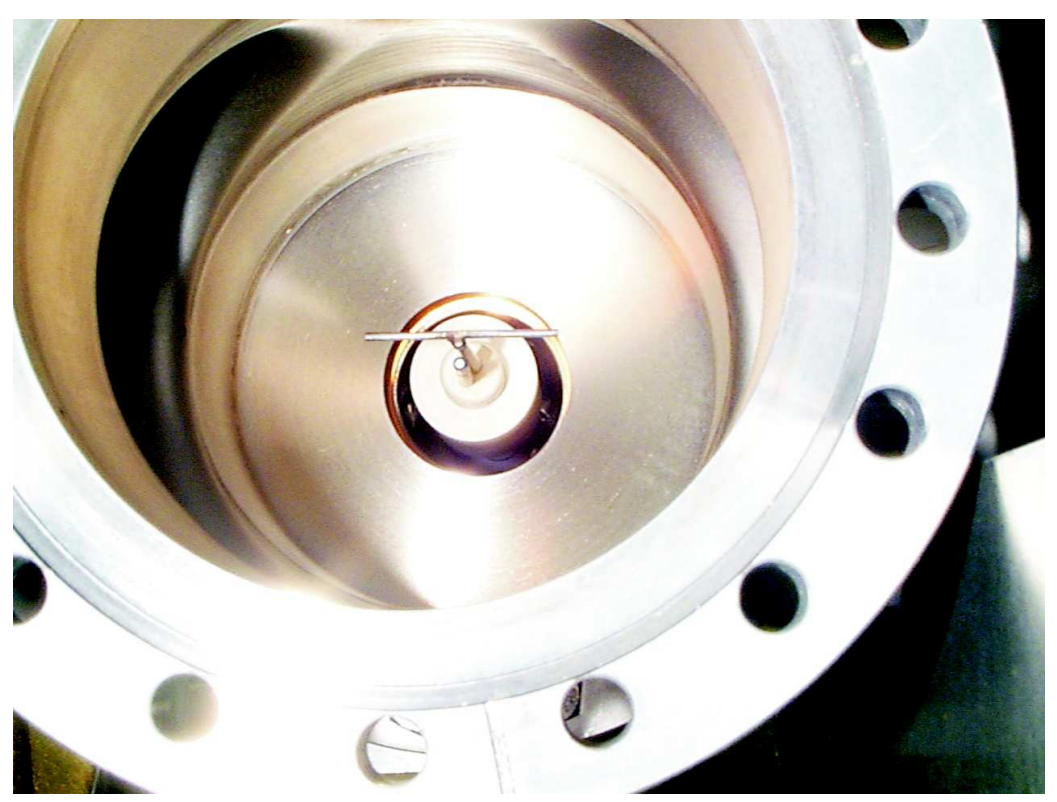

Figure 4.7: The Langmuir probe is shown as it sits inside the vacuum chamber. The probe head is perpendicular to the axis of the machine and to the dc magnetic field. The floating electrode is oriented along the dc magnetic field and is designed to track the changes in the plasma induced by the rf power.

Before the Langmuir probe was rf compensated, it was reporting electron temperatures in the order of $15 \mathrm{eV}$. After rf compensation, the electron temperature interpreted by the probe is about $6 \mathrm{eV}$. We have also verified this electron temperature using particle balance.

All this indicates that the Langmuir probe is well compensated in the context of our experiment.

The signal from the Langmuir probe is processed by a software package which we wrote in LabVIEW. The software processes the data for every plasma shot and returns the values of plasma density, temperature, floating potential, and plasma potential. The software uses two independent algorithms 


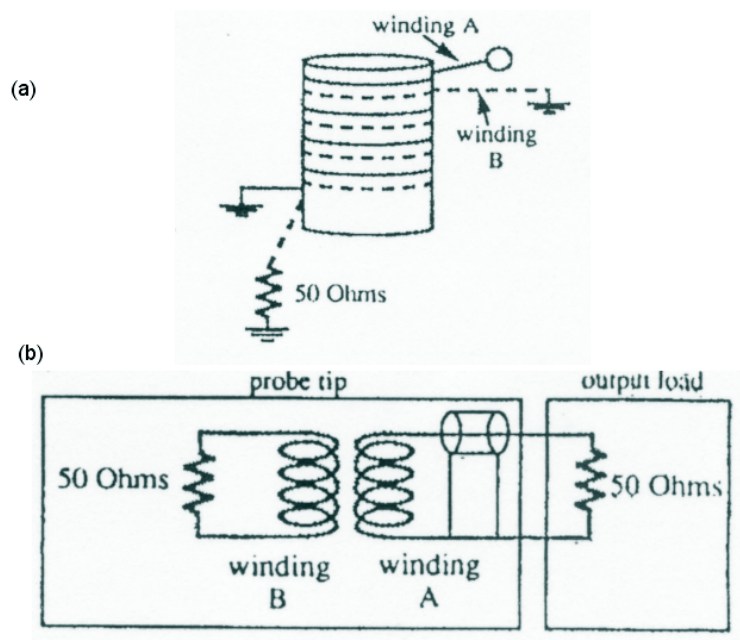

Figure 4.8: (a) Schematic diagram of a modified B-dot coil, (b) equivalent electrical circuit of a modified B-dot coil and connection to a $50 \Omega$ load (Loewenhardt, Blackwell, and Zhang, 1993).

for arriving at the plasma temperature. Their convergence gives us additional confidence in our results. The data acquisition process is triggered by the rf pulse. The software has a built-in delay of $100 \mathrm{~ms}$ after the beginning of the rf pulse before actual data collection starts. This ensures that the plasma has reached a steady state before data acquisition begins.

\subsection{Magnetic Probe}

The magnetic probe we are using is a modification of the center-tapped B-dot probe. It was developed for use in helicon discharges by Loewenhardt, Blackwell, and Zhang [25]. The probe consists of three mutually perpendicular 
coils for sampling $B_{r}, B_{\theta}$, and $B_{z}$. Each coil consists of two windings that are bifilar to each other but wound in opposite directions. Only winding A contributes to the measured inductive signal, but both windings contribute to the cancellation of electrostatic pickup signals. A gradient in the local plasma potential will result in a voltage drop across both windings but the resulting currents will be in opposite directions as can be seen from Fig. 4.8. Therefore the magnetic fields created by these currents will be in opposite directions and will cancel. The capacitive currents have to be equal in magnitude for the cancellation to be complete. That is why if winding B is connected to a $50 \Omega$ load, winding A has to be connected to a measuring equipment with a $50 \Omega$ input impedance. In our experiment we use spectrum analyzers to measure the magnetic signal and the $50 \Omega$ input impedance requirement is naturally satisfied. As cited in [25], this probe design shows a factor of ten improvement in electrostatic cancellation compared to the singly wound probe, and a factor of two improvement over a center-tapped probe. Fig. 4.9 shows the magnetic probe. The probe is in air, which helps with cooling, and is inserted inside a tight-fitting glass tube. The glass tube is partially inserted into the vacuum vessel through a vacuum seal.

As shown in Fig. 3.1 we are using two magnetic probes: one radial and close to the antenna, and one axial probe which can sample close to the antenna as well as downstream. 


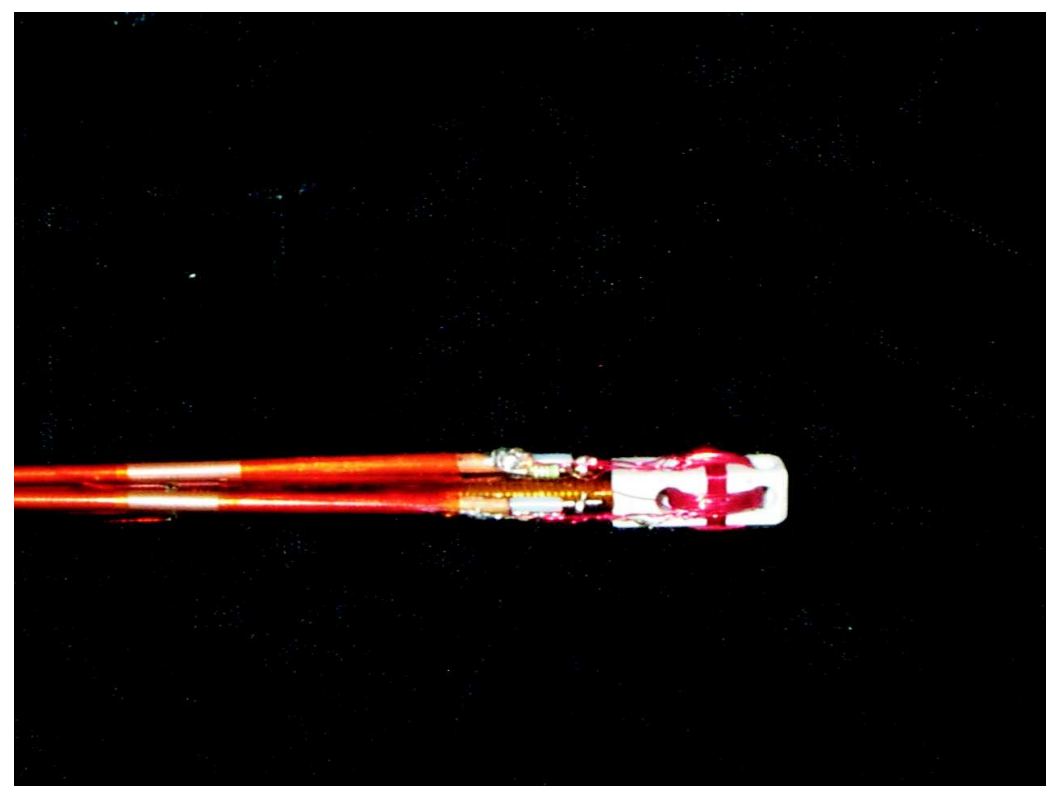

Figure 4.9: Shown is the actual magnetic probe. The probe head is made of boron nitride and the three mutually perpendicular coils are wrapped on it. The signal from each coil is transmitted through a rigid coaxial cable which adds structural strength to the whole probe assembly. 


\section{Chapter 5}

\section{Experimental results}

In this experiment we operate the helicon discharge under several distinct sets of experimental conditions some of which are summarized in Table 5.1. The plasma discharge exhibits the characteristic blue core which is the result of the $488 \mathrm{~nm}$ transition line of $\mathrm{Ar}^{+}$. The blue core of the helicon plasma in our experiment is about $2 \mathrm{~cm}$ in radius and about $45 \mathrm{~cm}$ in length. We also verified this transition line spectroscopically. Both experimental conditions produce high-density helicon discharges. However, the discharge at $627 \mathrm{G}$ and $6 \mathrm{mT}$ argon has a higher density and a steeper radial density gradient than the discharge produced at $313 \mathrm{G}$ and $4 \mathrm{mT}$ argon. Therefore, for the purposes of this article we call the first case a high-density discharge, and the second case a low-density discharge. These experimental conditions are chosen in order to create helicon plasmas with different densities and density gradients and to eliminate the Trivelpiece-Gould mode from consideration in our analysis of the data. 
Table 5.1: Two sets of parameters for the helicon discharge under investigation.

\begin{tabular}{lcr}
\hline \hline Gas & argon & argon \\
Base pressure & $5 \times 10^{-6}$ Torr & $5 \times 10^{-6}$ Torr \\
Fill pressure & $4 \mathrm{mTorr}$ & $6 \mathrm{mTorr}$ \\
Fixed frequency power & $1 \mathrm{~kW}$ & $1 \mathrm{~kW}$ \\
Fixed frequency & $13.56 \mathrm{MHz}$ & $13.56 \mathrm{MHz}$ \\
Variable frequency power & $10-15 \mathrm{~W}$ & $10-15 \mathrm{~W}$ \\
Variable frequency range & $9-15 \mathrm{MHz}$ & $9-15 \mathrm{MHz}$ \\
Magnetic field & $627 \mathrm{Gauss}$ & $313 \mathrm{Gauss}$ \\
Length of rf pulse & $1000 \mathrm{~ms}$ & $1000 \mathrm{~ms}$ \\
\hline \hline
\end{tabular}

\section{$5.1 \quad$ Langmuir probe results}

The plasma density and temperature at a fixed axial point are shown in Fig. 5.1. The Langmuir probe is immersed in the helicon discharge at this location. The discharge itself is about $45 \mathrm{~cm}$ long from the center of the antenna and $2 \mathrm{~cm}$ in radius. Visually, the strong $488 \mathrm{~nm} \mathrm{Ar}^{+}$emission line produces the intense blue light characteristic of helicons in argon.

Fig. 5.2 shows the plasma density outside of the active helicon discharge. At that point, the plasma is tenuous and visually can be seen only due to the excitation collisions of electrons with neutrals as the electrons stream axially. Previous analysis [1] shows that the ions escape radially with the $\vec{E} \times \vec{B}$ drift velocity. The electrons are lost axially accelerated by $E_{\|}$where $E_{\|} \approx \frac{T_{e}}{|e| L}$. This velocity is comparable to the electron thermal velocity.

It is also clear that the $313 \mathrm{G}$ discharge has a smaller density gradient than the $627 \mathrm{G}$ discharge. The significance of that result will be explored in the next section. Most importantly, the Langmuir probe data shows that the helicon 


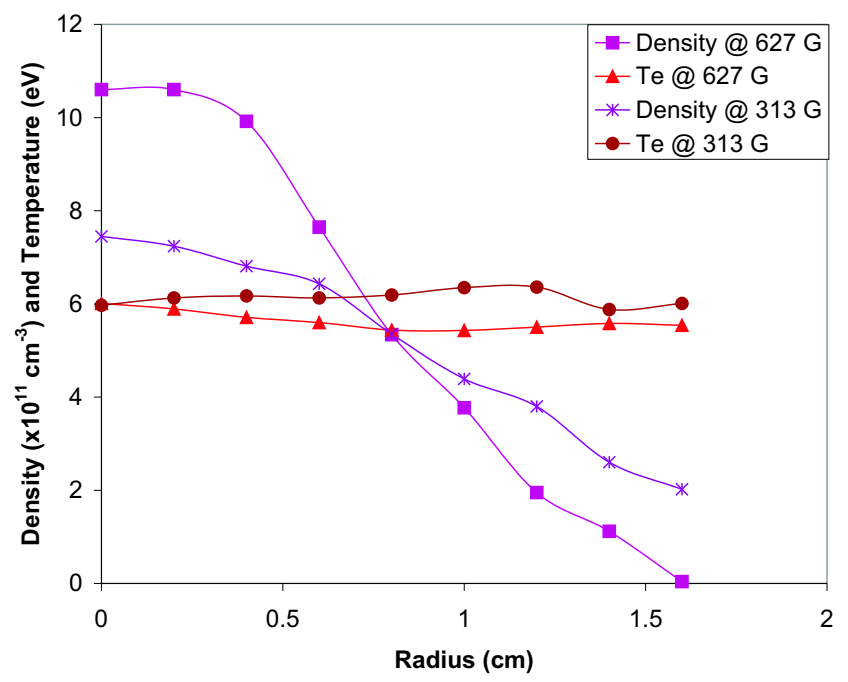

Figure 5.1: Density and temperature measured by the rf compensated Langmuir probe $30 \mathrm{~cm}$ downstream from the center of the antenna.

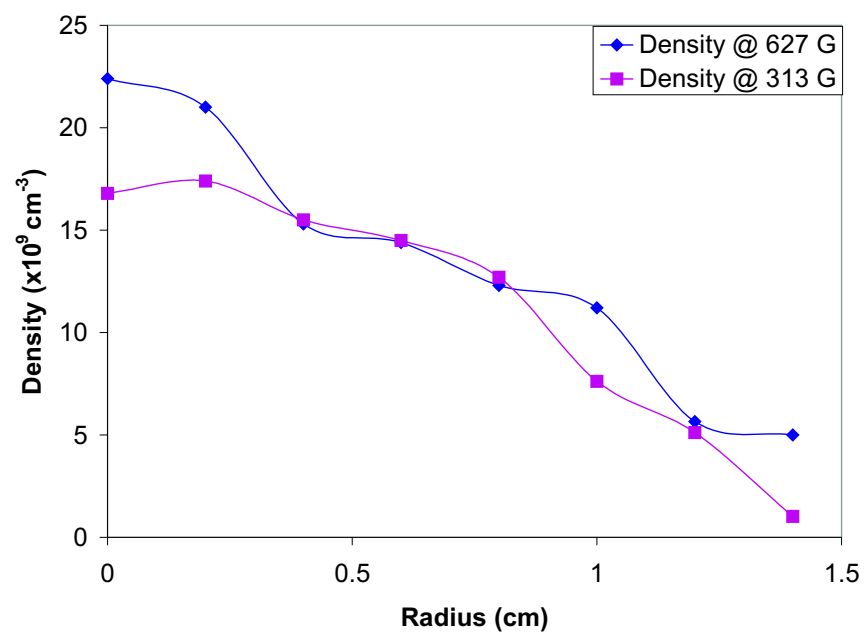

Figure 5.2: Density measured by the Langmuir probe $84 \mathrm{~cm}$ downstream from the center of the antenna. 
plasma has a strong density gradient both in the radial and axial directions.

The Langmuir probe data also shows clear evidence for the existence of fast non-Maxwellian electrons in the discharge. We will present the data and analysis of that aspect of the helicon plasma in Chapter 7.

\subsection{Magnetic probe data}

The profile of the $B_{r}$ component of the wavefield is shown in Fig. 5.3 for the high-density plasma discharge at $627 \mathrm{G}$. The data is collected with the radial megnetic probe at a fixed axial location $30 \mathrm{~cm}$ downsream from the center of the antenna. It reveals a specific profile for the amplitude of $B_{r}$ throughout the frequency range which points to the existence of a universal mode in the helicon plasma. Such magnetic profiles are contradictory to the ones predicted by the uniform density treatment of the plasma and have been observed before but never explained [19]. These profiles are consistent with the theory of Breizman and Arefiev [9] and represent an important clue to the underlying structure of the resonant 7helicon mode. We will come back to this point in the analysis section. Part of the problem in detecting these profiles is that up until now the magnetic data has been collected only at the main driving frequency. These profiles are evident when a broader frequency range is explored. One of the difficulties in detecting the same mode at the driving frequency is the strong electrostatic pickup at that frequency. Although our B-dot probes reject that pickup by design, we had to do additional electrostatic shielding at the driving $13.56 \mathrm{MHz}$ frequency. 


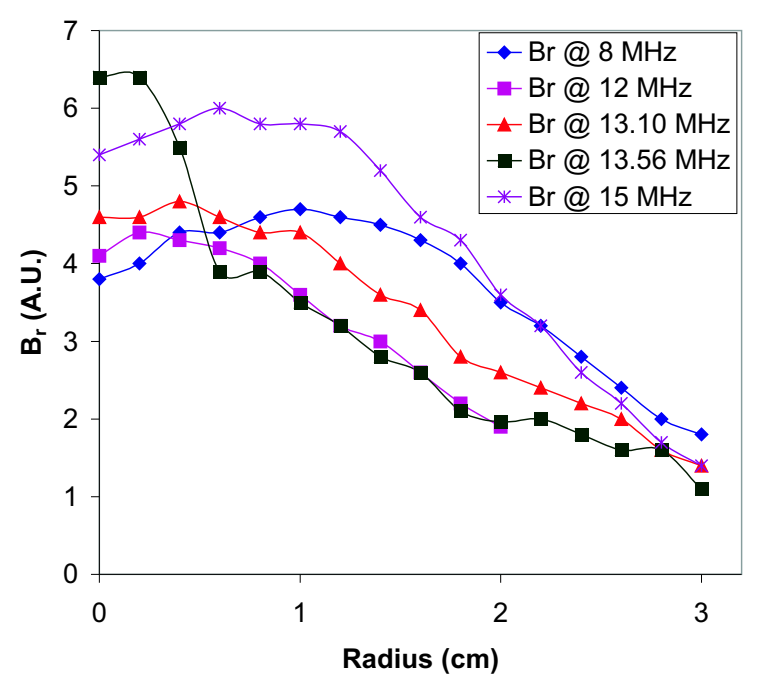

Figure 5.3: Amplitude profiles of the $B_{r}$ component of the electromagnetic wave taken at a fixed axial location within the helicon discharge for the highdensity case. The amplitude of $B_{r}$ at the driving frequency is scaled to fit on the plot.

Fig. 5.4 shows the three magnetic components of the electromagnetic helicon wave for the lower-density discharge at $313 \mathrm{G}$. The $B_{r}$ component shows the same pattern as in Fig. 5.3 but not as pronounced. Our data also shows the same pattern throughout the frequency range but somewhat attenuated compared to the high-density case. It is known that in the uniform density theory of helicon discharges [13] the profile of $B_{r}$ is a decreasing function of $r$ for small $r$. Clearly, our magnetic profile results cannot be reconciled with that theory. We will come back to this point later. On the other hand, the $B_{z}$ and $B_{\theta}$ components for both high-density and low-density cases in our experiment exhibit the usual $m=+1$ profiles that have been detected by various other 


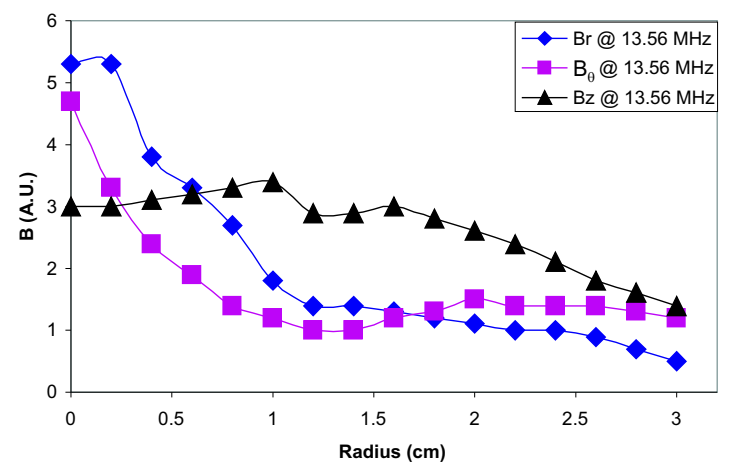

Figure 5.4: Profiles of the magnetic field amplitude of the helicon electromagnetic wave taken at a fixed axial location within the helicon discharge for the low-density case.

researchers. (Note that the magnetic data here shows the amplitude of the wave magnetic field, not the phase.)

The distribution of the wave magnetic induction $B$ in the plasma chamber is determined using the axial and the radial B-dot probes scanning at the driving 13.56 $\mathrm{MHz}$ frequency. In the axial direction the total magnitude of the wave magnetic field is plotted versus the axial position and then fitted to a function of the form $B=B_{0} \exp \left(-z / L^{*}\right)$ where $L^{*}$ is a characteristic decay length. Fig. 5.5 shows the results for the high-density ( $627 \mathrm{G} 6 \mathrm{mT}$ argon) case. The characteristic decay length of the induced magnetic field is $L^{*} \approx 17 \mathrm{~cm}$. We obtained similar results for the low-density case.

In order to obtain the amplitude of the magnetic induction in the radial dimension, we collapse the vector components of $\vec{B}$ obtained from the radial B-dot probe to form the scalar amplitude $B=\left(B_{r}^{2}+B_{\theta}^{2}+B_{z}^{2}\right)^{1 / 2}$. It turns 


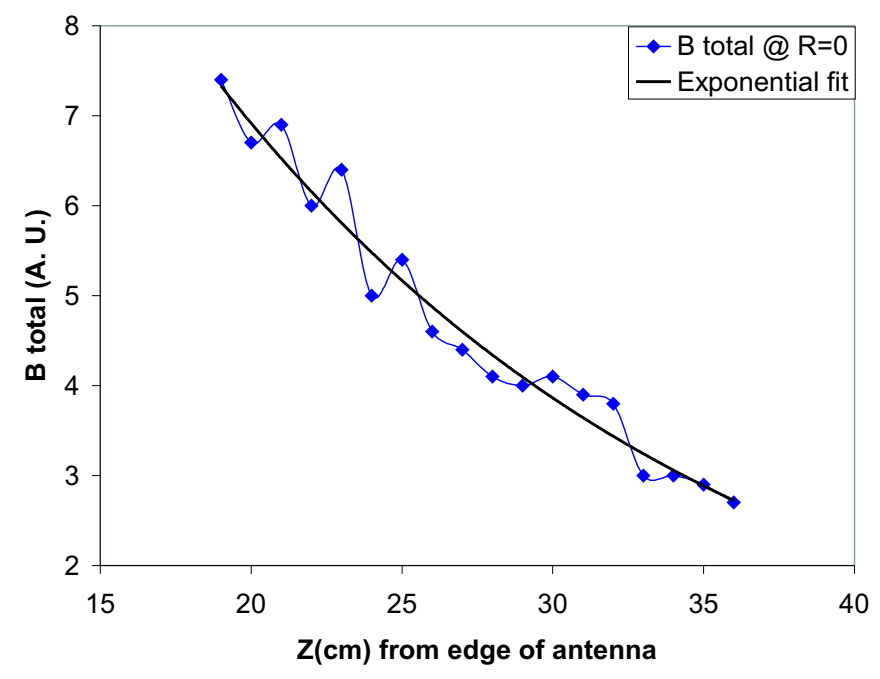

Figure 5.5: Axial dependance of the total wave magnetic field amplitude in the high-density helicon discharge.

out experimentally that the resultant amplitude is closely approximated by an exponential decay function. We obtain that the characteristic radial decay length for the high-density case is $R^{*} \approx 1.9 \mathrm{~cm}$ and $R^{*} \approx 1.7 \mathrm{~cm}$ for the lowdensity case. This allows us to combine the results from the radial and axial magnetic probes and model the magnetic induction amplitude as

$$
B(r, z)=B_{0} \mathrm{e}^{-z / L^{*}} \mathrm{e}^{-r / R^{*}}
$$

where $B_{0} \approx 14.0 \mathrm{G}$ for the high-density case, and $B_{0} \approx 14.5 \mathrm{G}$ for the lowdensity case. Fig. 5.6 shows a 3-D plot of Eq. 5.1 for the high-density case. The magnetic induction amplitude $B(r, z)$ will allow us to calculate the magnetic energy in the discharge and the amount of power that the helicon mode deposits into the plasma, provided we have an estimate for the damping rate. 


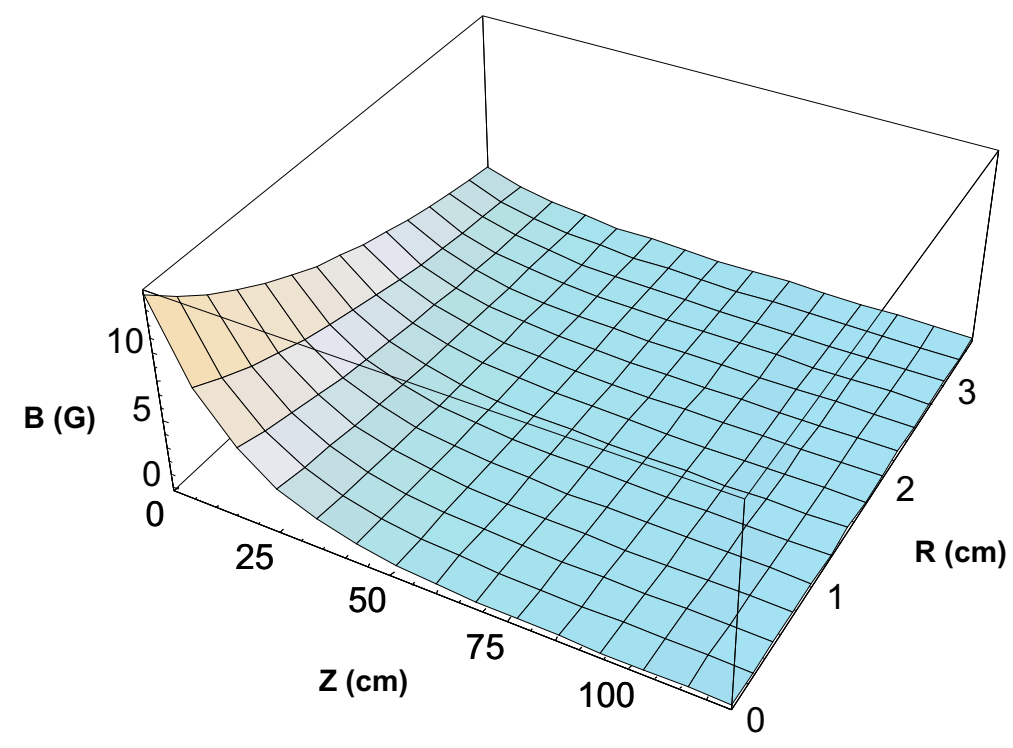

Figure 5.6: Wave magnetic field amplitude in the helicon chamber. The axial direction is measured from the edge of the antenna.

\subsection{Plasma impedance data}

Using the procedure outlined in Section 4.1 the rf sensor which measures voltage, current, and phase angle scans the plasma impedance as a function of frequency. The data gave a good indication that there is a resonance of the plasma impedance near $13.20 \mathrm{MHz}$. Both the real part and the imaginary part of the impedance exhibit behavior similar to the amplitude response of a mechanical oscillator with damping subjected to an outside driving force. Because of engineering considerations, however, the location of the rf sensor in the system is not optimal to detect the subtle changes in the plasma 
impedance. Therefore, the results from the rf sensor are best described as qualitative, not quantitative. Nevertheless, this was an important result since it contains the full impedance information - voltage, current, and phase. It allows us to conclude that the imaginary response of the plasma impedance is much stronger than the real part. This allows us to model the plasma response accordingly and in a sense reduce the problem to a one-dimensional treatment. Then using a sensitive current meter placed directly on the antenna, our calibration database, and Eq. 4.1b we can determine the plasma impedance in the frequency range of interest. The signal from the current meter is read by a spectrum analyzer, giving a clear distinction between the current at the 13.56 $\mathrm{MHz}$ driving frequency and the current from the active frequency probe. Once the problem is one-dimensional, this method has a clear advantage over the rf sensor method because it is much easier to accurately measure current instead of phase angle in the noise-filled environment of the helicon discharge. The results are presented in Fig. 5.7. They confirm and render more precise the results from the rf sensor. Clearly, there is a resonance of the plasma impedance for both the low-density discharge in our experiment, as well as for the high-density discharge. The resonance is close to the driving $13.56 \mathrm{MHz}$ frequency. Most importantly, these results show that the plasma operates under conditions of resonant power absorption.

We have not detected any additional plasma resonances in the $9-15 \mathrm{MHz}$ range.

We now have all the components we need: plasma impedance data, mag- 


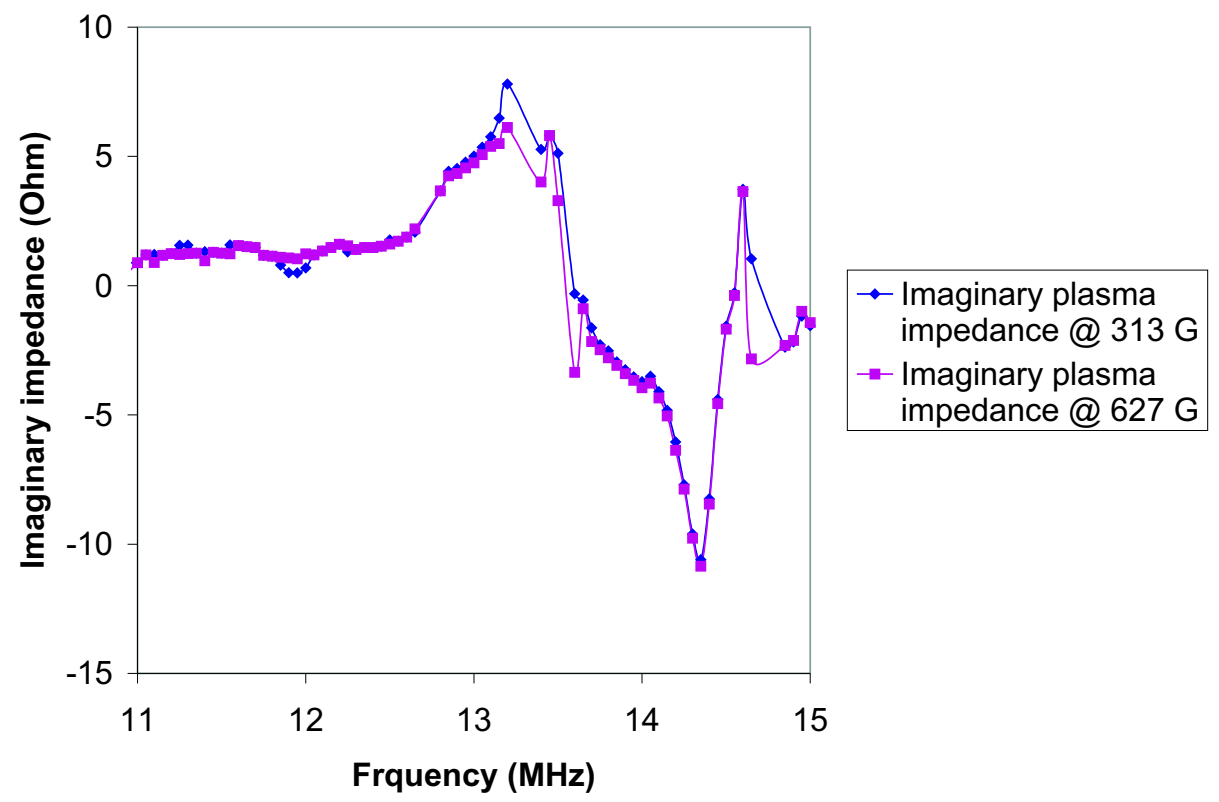

Figure 5.7: Imaginary component of the plasma impedance showing a resonance response close to the driving frequency. 
netic radial and axial profiles, damping lengths, and density and temperature profiles, to try to give a coherent explanation of the observed experimental results. 


\section{Chapter 6}

\section{Analysis}

\subsection{Plasma resonance}

We investigate the observed plasma resonance using the model presented in Fig. 6.1. The matrix equations for the transformer formed by the antenna and the resonant plasma are

$$
\begin{aligned}
& V_{r f}=\mathrm{i} \omega L_{11} I_{r f}+\mathrm{i} \omega L_{12} I_{p} \\
& V_{p}=\mathrm{i} \omega L_{21} I_{r f}+\mathrm{i} \omega L_{22} I_{p} \\
& V_{p}=-I_{p}\left(R_{p}+\frac{I_{p}}{\mathrm{i} \omega C_{p}}\right)
\end{aligned}
$$

where $L_{22} \equiv L_{p}$. Solving these equations for $I_{p}$ we obtain

$$
I_{p}=\frac{\mathrm{i} \omega L_{21} I_{r f}}{\frac{\mathrm{i}}{\omega C_{p}}-R_{p}-\mathrm{i} \omega L_{p}}
$$

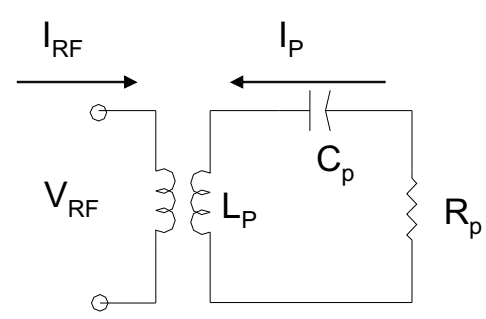

Figure 6.1: Equivalent circuit for the plasma resonance model. 
Here $I_{p}$ is analogous to displacement of a forced harmonic oscillator. Using the notation for a mechanical harmonic oscillator we rewrite Eq. 6.2 in the following form

$$
I_{p}=\frac{\omega^{2} L_{21} I_{r f} / L_{p}}{\omega_{0}^{2}-\omega^{2}+2 \mathrm{i} \gamma \omega}
$$

where $L_{21}$ is the mutual inductance between the antenna and the plasma, $\omega_{0}$ is the resonance frequency, and $\gamma$ is the damping rate. This equation corresponds directly to the expression for a mechanical oscillator, where $I_{p}$ relates to a mechanical displacement, and $I_{r f} / L_{p}$ corresponds to the $\frac{\text { Force }}{\text { mass }}$ term driving the oscillation. However, experimentally we are not measuring $I_{p}$ but the impedance seen at the antenna terminals. That impedance is given by

$$
Z=\frac{V_{r f}}{I_{r f}}=\mathrm{i} \omega L_{11}+\frac{\omega^{2} L_{12}^{2}}{R_{p}+\mathrm{i}\left(\omega L_{p}-\frac{1}{\omega C_{P}}\right)}
$$

The first term in Eq. 6.4 is simply the impedance of the antenna itself. The second term is the plasma impedance $Z_{p}$. Since from the rf sensor we know that the plasma impedance is predominantly imaginary, we concentrate on the imaginary component of $Z_{p}$. That component is given by

$$
\operatorname{Im}\left[\frac{\omega^{2} L_{12}^{2}}{R_{p}+\mathrm{i}\left(\omega L_{p}-\frac{1}{\omega C_{p}}\right)}\right]=\frac{\omega^{2} L_{12}^{2}\left(\frac{1}{\omega C_{p}}-\omega L_{p}\right)}{R_{p}^{2}+\left(\frac{1}{\omega C_{p}}-\omega L_{p}\right)^{2}}
$$

Rewriting this in terms of the resonance parameters we obtain

$$
\operatorname{Im}\left[Z_{p}\right]=\frac{\omega^{3} \frac{L_{12}^{2}}{L_{p}}\left(\omega_{0}^{2}-\omega^{2}\right)}{4 \omega^{2} \gamma^{2}+\left(\omega_{0}^{2}-\omega^{2}\right)^{2}}
$$

We then fit Eq. 6.6 to the data presented in Fig. 5.7 to obtain the resonance parameters. The plot of the curve fit and the experimental data being fitted are 


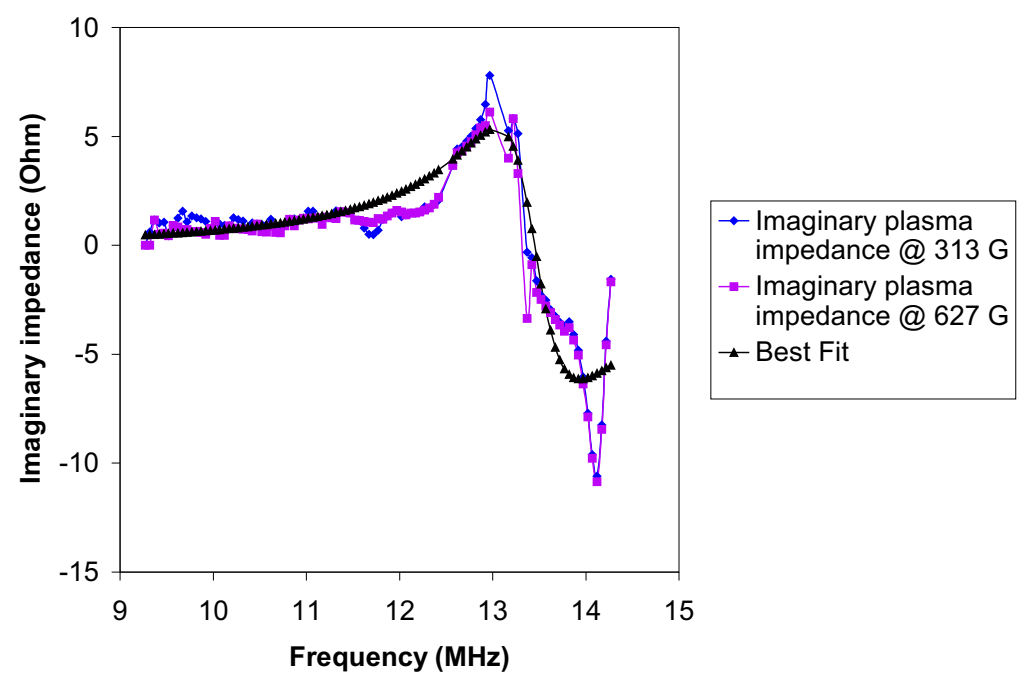

Figure 6.2: Curve fit of the imaginary component of the plasma impedance plotted together with the experimental data being fitted.

shown in Fig. 6.2. The resonance parameters are summarized in Table 6.1. The expression for the real component of $Z_{p}$ in terms of the resonance parameters is given by

$$
\operatorname{Re}\left[Z_{p}\right]=\frac{2 \omega^{4} L_{12}^{2} / L_{p} \gamma}{4 \omega^{2} \gamma^{2}+\left(\omega_{0}^{2}-\omega^{2}\right)^{2}}
$$

Using the values given in Table 6.1 we can plot Eq. 6.7 for the real component of $Z_{p}$. The result is given in Fig. 6.3 and gives the real component of the plasma impedance calculated using the experimentally determined values of the resonance parameters.

We have also performed a theoretical estimate of the real component of the plasma impedance $R_{s}$ following a procedure outlined in [22]. The result is that for the low-density discharge $R_{s} \approx 2 \Omega$, and for the high-density dis- 
Table 6.1: Resonance parameters.

\begin{tabular}{lr}
\hline \hline Driving frequency & $13.56 \mathrm{MHz}$ \\
Resonance frequency $f_{0}=\omega_{0} / 2 \pi$ & $13.45 \mathrm{MHz}$ \\
Damping frequency $\nu_{\text {damping }}=\gamma / 2 \pi$ & $3.82 \times 10^{5} \mathrm{~s}^{-1}$ \\
Chi-Square goodness of fit & 900 \\
\hline \hline
\end{tabular}

charge $R_{s} \approx 2.3 \Omega$. These results are consistent with Fig. 6.3.

The most important experimental results stemming from the plasma impedance data are:

- There exists a resonant electromagnetic mode in the helicon discharge in the frequency space close to the driving frequency.

- The resonant damping rate is much smaller than the driving frequency. The Q-factor of the resonance is approximately 17 .

- The resonance frequency is lower than the driving frequency as is required in general for a steady state operation (See Section 6.7).

One immediate consequence of these results is that we can form the ratio of the driving frequency $f_{\text {driving }}=13.56 \times 10^{6} \mathrm{~s}^{-1}$ and the resonant damping rate $\nu_{\text {damping }}=3.82 \times 10^{5} \mathrm{~s}^{-1}$. That ratio is $\frac{13.56 \times 10^{6} \mathrm{~s}^{-1}}{3.82 \times 10^{5} \mathrm{~s}^{-1}} \approx 35$ and gives the average number of wavelengths that the electromagnetic mode needs to deposit energy into the plasma. At 35 wavelengths the required distance is about $10 \mathrm{~m}$ which is well beyond the length of the discharge and even beyond the size of the helicon machine. In order to offer a coherent explanation, we need to mention some of the central features of the theory developed by Breizman and Arefiev $[1,9]$. 


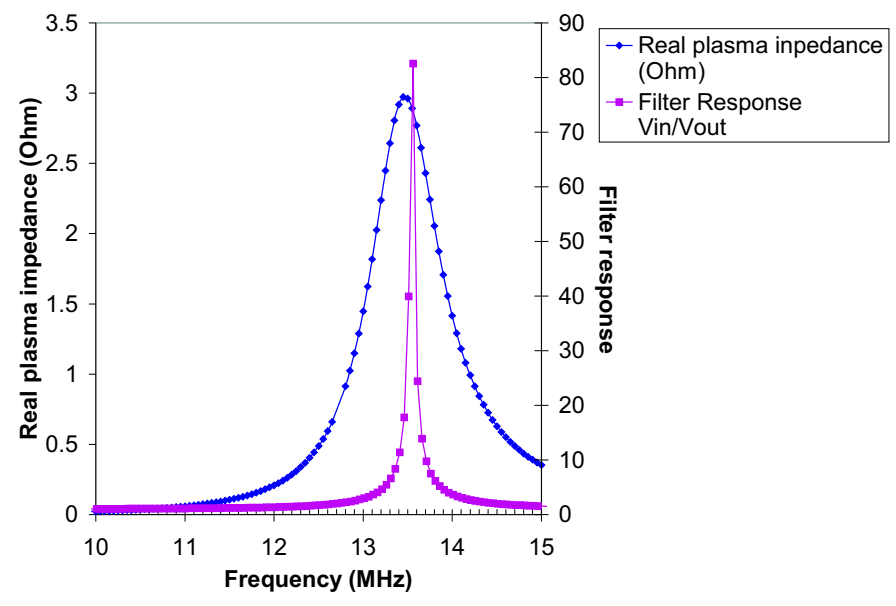

Figure 6.3: Real component of the plasma impedance calculated from Eq. 6.7 using the experimental values of the resonance parameters. Also shown is the frequency response of the blocking LC filter. The halfwidth of the filter is less than $1 / 10$ the halfwidth of the resonance curve.

\subsection{Theoretical summary}

A function is defined given by

$$
E=E_{\|}-\frac{k_{\|} r}{m} E_{\phi}
$$

which relates to the radial component of the wave magnetic field as

$$
H_{r}=\frac{c m}{\omega r} E
$$

where $m$ is the mode number. This function satisfies the relationship

$$
E=E_{\|}\left[1+k_{\|}^{2} r \eta\left(m \frac{\partial g}{\partial r}\right)^{-1}\right]
$$

where

$$
g=\frac{\omega_{p e}^{2}}{\omega \omega_{c e}}, \quad \eta=-\frac{\omega_{p e}^{2}}{\omega^{2}}, \quad \omega_{c e}=\frac{e B}{m_{e} c}
$$


and $\omega_{c e}$ is defined with an implicitly negative electric charge $e$. With some simplifications this leads to

$$
\frac{1}{r} \frac{\partial}{\partial r}\left[r \frac{\partial E}{\partial r}\right]-\frac{m^{2}}{r^{2}} E=-\frac{m}{k_{\|}^{2} r} \frac{\omega^{2}}{c^{2}} E \frac{\partial g}{\partial r}
$$

This is the wave equation for the helicon resonance eigenmode. Its physical meaning is revealed in the next section. Eq. 7.4 also reveals a peripheral feature of the helicon eigenmode. As is pointed out in [9], Eq. 7.4 has a singularity in $E_{\|}$when the term in brackets becomes zero and $E$ is finite. This creates the condition for a resonant power absorption at the singularity. The resonant condition on the parallel electric field suggests that a strong parallel electric field will be present in the plasma. This effect is related to the presence of non-Maxwellian electrons in the discharge and will be treated in Chapter 7 .

The steady-state regime for this electromagnetic mode requires the resonant frequency to be lower than the driving frequency $[1,29]$, which is in general a condition for the steady-state operation at a near-resonance frequency. It is shown that the plasma self-adjusts when the discharge is ignited in such a way that this condition is fulfilled. We discuss the stability criterion in section 6.7 .

\subsection{Physical mechanism of the helicon discharge}

To reveal the physical meaning of Eq. 6.12 we write it [1] as a onedimensional Schrödinger equation for a particle in a potential well:

$$
\frac{\partial^{2} E}{\partial s^{2}}+\left[\left(-m^{2}\right)-U(s)\right] E=0
$$




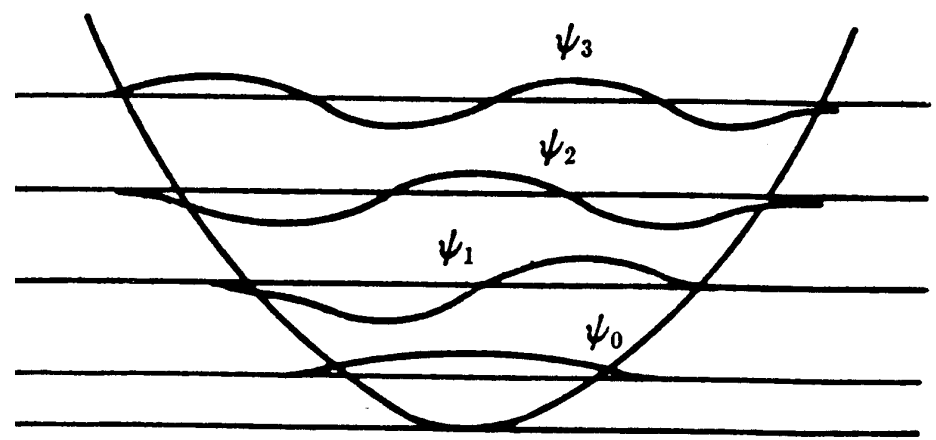

Figure 6.4: State functions and energy levels in a quantum well. This corresponds to a potential cavity formed by the density gradient in the helicon discharge. The state function $\psi$ is replaced by $E$ in the plasma case.

where $s \equiv \ln r$ and

$$
U \equiv-\frac{m r}{k_{\|}^{2} r} \frac{\omega^{2}}{c^{2}} \frac{\partial g}{\partial r}
$$

Eq. 6.13 is mathematically identical to a one-dimensional Schrödinger equation and has the same solutions. In this context the state function $\psi$ is replaced by $E, U(s)$ is the potential energy, and $\left(-m^{2}\right)$ is the particle energy. If the plasma density gradient $\partial n / \partial r=0$, then $U(s)=0$. If the plasma density gradient $\partial n / \partial r<0$, as is the case in our experiment and in all helicon experiments where $m=+1$, then $U(s)<0$ and the density gradient forms a potential well in the radial direction. Then the state function $E$ is trapped in that potential well as shown in Fig. 6.4. The physical meaning behind this picture is that the electromagnetic energy launched by the antenna is confined radially by a density gradient. It also means that given the same experimental conditions, 
the sign of $m$ controls if the density gradient will form a potential well or not. If a well is not formed, then the resonant plasma mode that we are describing here will not exist. Reversing the sign of $m$ requires a reversal in the direction of the dc magnetic field.

We reversed the field and similarly to the results in numerous other studies, we observed that the plasma discharge was weaker. The usual explanation given in the literature is that the antennas that have a helical twist are incapable of coupling to the $m=-1$ helicon mode. The implication is that the antenna geometry prevents them from launching that mode. However, intensive studies of the most widely used helicon antenna - the Nagoya antenna [23], have revealed that this antenna also fails to launch the $m=-1$ mode. This is puzzling since geometrically the Nagoya antenna is symmetric with respect to the $m=+1$ and $m=-1$ modes and to the direction of the dc magnetic field. We offer an alternative explanation. Considering the fact that helicon discharges also have an axial density gradient, it is a natural conclusion to suggest that the same mechanism can be used to trap the electromagnetic wave in the axial direction as well. Currently the theory is being extended in that respect. In the $m=+1$ mode the wave energy is trapped and although the damping rate is relatively low, the wave bounces back and forth, continuously depositing energy into the plasma. Reversing the sign of $m$ from $m=+1$ to $m=-1$ replaces the potential well with a potential barrier as can be seen in Eq. 6.14. As a result the electromagnetic wave is not trapped and leaves the system in a single pass. Because the damping rate is relatively weak, the 
amount of energy that the wave deposits in a single pass is too low to sustain the discharge.

In the context of an oscillator in a potential well $(m=+1$ mode), it is reasonable to assume that the ground state mode is the one that is the easiest to excite and that is the mode that we are detecting. This suggests that the axial dependance of the wave magnetic field amplitude presented in Fig. 5.5 is actually a portion of the ground state function $\psi_{0}$ (in our case $E_{0}$ ) of a damped harmonic oscillator in a potential well as shown in Fig. 6.4.

Some researchers have suggested $[17,18]$ that the fast energy damping in helicon discharges is due to electron trapping in the helicon wave. This mechanism has two immediate consequences which are within the scope of our experiment. The first corollary is that there will be a significant non-Maxwellian electron population in the discharge. Our experimental results on that aspect of the discharge are presented in Chapter 7 and show that the number density and significance of the non-Maxwellian electron population in our experiment is only marginal. Moreover, the electron trapping will be manifest in the plasma impedance and again our data does not support that. Our experimental results seem to rule out this highly nonlinear damping mechanism.

\subsection{Wave equation and magnetic profiles}

Our experimental results seem to confirm the validity of this model: the experimental verification of a resonance condition in the helicon plasma, the fact that the resonant frequency is slightly lower than the driving frequency, 


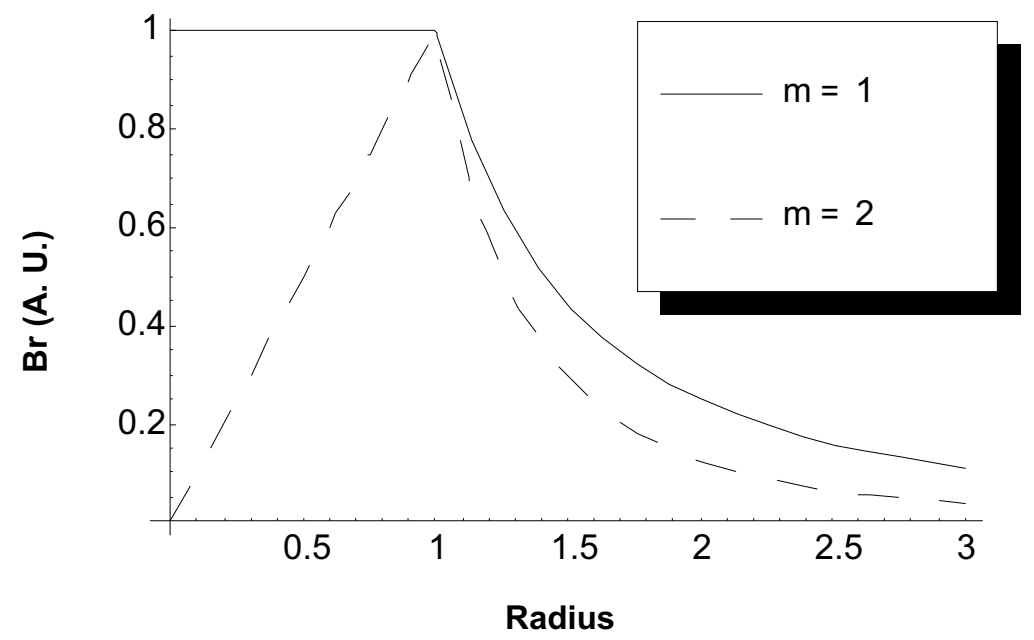

Figure 6.5: Approximate theoretical profiles of $B_{r}$ predicted by the radially localized resonant theory of helicon discharges.

and the shape of the radial component of the wave magnetic field $B_{r}$ over the entire frequency range as presented in Fig. 5.3 and Fig. 5.4. For plasmas with a strong density gradient, an approximate solution of Eq. 6.12 predicts that the $B_{r}$ component of the wave field will have the shape as shown in Fig. 6.5 for $m=+1$ and $m=+2$ modes [9]. There is a qualitative agreement between our experimental data and the approximate solution for the $m=+1$ mode. This also explains why our $313 \mathrm{G}$ discharge with its lower density gradient has profiles of $B_{r}$ that are less peaked than the profiles of the high-density gradient discharge at $627 \mathrm{G}$. We were also able to solve the wave equation (Eq. 6.12) exactly. The solution is a linear combination of Bessel functions and is given 
by

$$
E=A J_{2}\left\{\frac{2 \omega}{k_{\|} c}\left(m r \frac{\partial g}{\partial r}\right)^{1 / 2}\right\}+B N_{2}\left\{\frac{2 \omega}{k_{\|} c}\left(m r \frac{\partial g}{\partial r}\right)^{1 / 2}\right\}
$$

where $J_{2}$ is a Bessel function of the first kind of order two, $N_{2}$ is a Bessel function of the second kind of order two, and $E=\frac{\omega r}{c m} B_{r}$. Using our plasma density data for the high-density discharge, we obtain an estimate for $\partial g / \partial r$ and using our magnetic probe data we can estimate Eq. 6.15 to obtain the values of the constants $A$ and $B$. The solution is

$$
\begin{array}{lll}
B_{r}=\frac{1}{r} 7.0 J_{2}\left\{1.6 r^{1 / 2}\right\}+\frac{1}{r} 0.1 N_{2}\left\{1.6 r^{1 / 2}\right\} & \text { for } & m=+1 \\
B_{r}=\frac{1}{r} 5.6 J_{2}\left\{2.3 r^{1 / 2}\right\}+\frac{1}{r} 1.8 N_{2}\left\{2.3 r^{1 / 2}\right\} & \text { for } & m=+2
\end{array}
$$

A plot of these solutions is shown in Fig. 6.6. In order to obtain a solution, we have treated $\partial g / \partial r$ as a parameter. Therefore, the presented solution is valid for $r>0.5 \mathrm{~cm}$. Looking at Fig. 5.3 and Fig. 5.4 we can conclude that the modes present in our experiment are $m=+1$ and $m=+2$ with the $m=+1$ mode dominating. Fig. 6.7 shows the magnetic profile for $B_{r}$ predicted by the preexisting helicon theory based on a uniform density profile. Comparing our experimental and theoretical results to Fig. 6.6 and Fig. 6.7 shows that the our data cannot be reconciled with the magnetic profiles based on the uniform density model, while there a good agreement with the magnetic profiles predicted by the plasma resonance model. Our experimental results and the plasma resonance model suggest that $m=+1$ is the dominant mode in our discharge. Although we have not measured directly the mode number in the discharge, based on numerous other helicon experiments and given the 


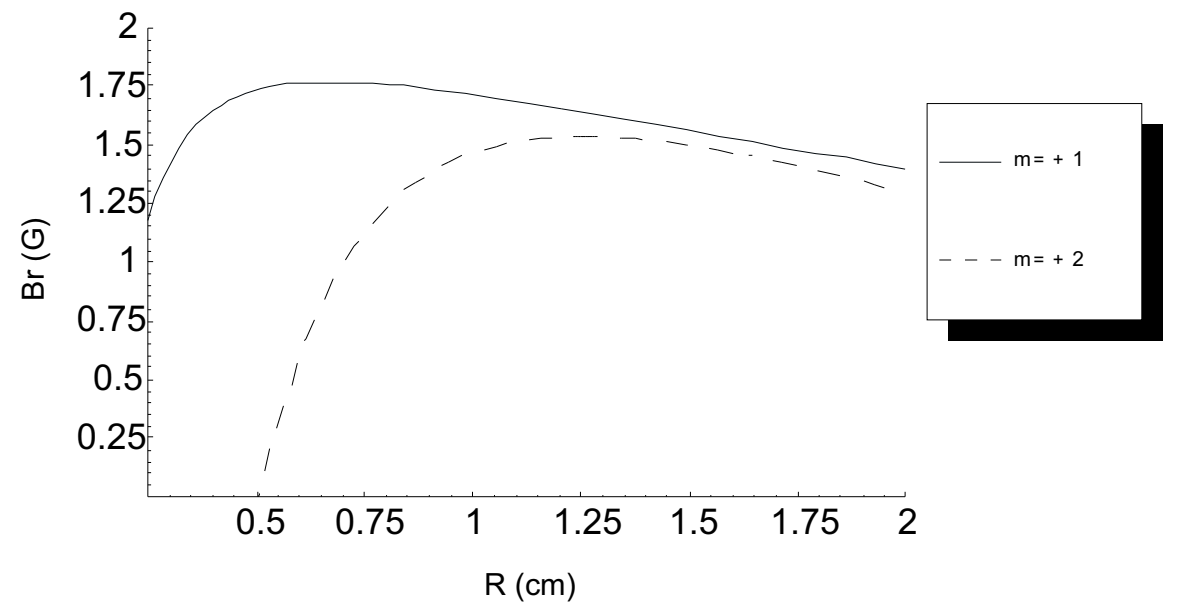

Figure 6.6: Exact solutions for the radial component of the wave magnetic field for the $m=+1$ and $m=+2$ modes based on the radially localized helicon mode theory.

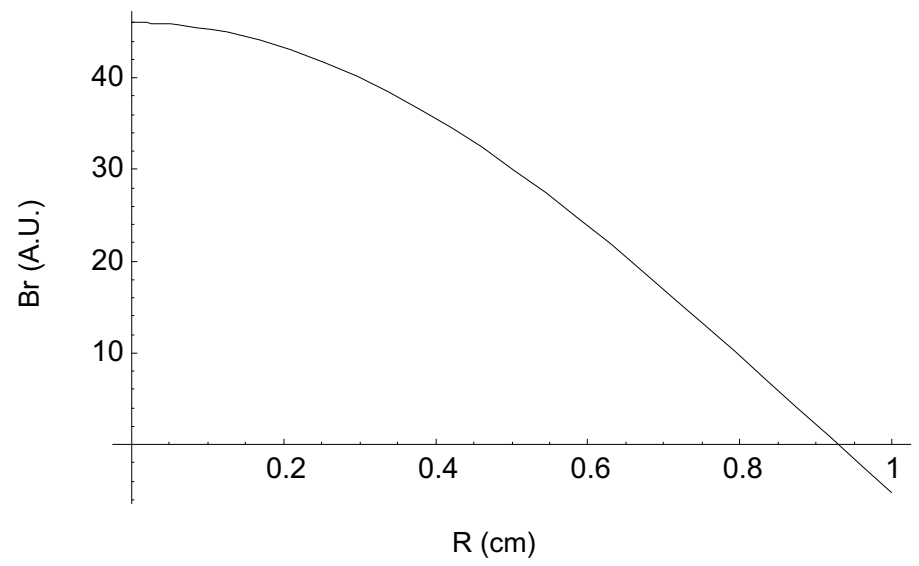

Figure 6.7: Radial component of the wave magnetic field as predicted by the uniform plasma theory. 
antenna we are using and the direction of the dc magnetic field, $m=+1$ is the mode number we expect which is a further confirmation of the consistency of the theoretical picture.

\subsection{Power flow analysis}

In order to determine the relative importance of this resonant electromagnetic mode for the helicon discharge, we calculate the power that the mode deposits into the plasma. The total power delivered to the plasma by the helicon eigenmode is given by

$$
P=I_{r f}^{2} \operatorname{Re}\left[Z_{p}\right] \quad \text { where } \quad I_{r f} \text { is in RMS. }
$$

The measured current through the antenna is $I_{r f} \approx 20 \mathrm{~A}$ while $\operatorname{Re}\left[Z_{p}\right]$ is the real component of the plasma impedance of the helicon eigenmode and $R_{s} \approx 2.6 \Omega$. This gives $1040 \mathrm{~W}$ of total deposited power into the plasma. The rf source puts out a little over $1 \mathrm{~kW}$ of power at every pulse and therefore, virtually all of the available rf power is deposited into the plasma by the helicon eigenmode.

Using the previous result and an alternative expression for the power deposited by the mode into the discharge, we can also estimate the energy stored in the helicon wave. The power deposited by the mode is given by

$$
P=2 \gamma W
$$


where $W$ is the energy stored in the helicon wave and $\gamma$ is the experimentally determined energy damping rate. This gives $W \approx 0.2 \mathrm{~mJ}$.

However, the overriding conclusion stemming from the power flow analysis is that the resonant helicon mode is the dominant mechanism which sustains the helicon discharge.

\subsection{Energy cost per electron-ion pair created}

We want to find out what is the energy cost of creating an electron-ion pair in our discharge and what fraction of that energy is electron energy loss due to ionization and electron energy loss due to excitation. To do this we need to estimate the power balance for our particular discharge.

The collisional energy $\mathcal{E}_{c}$ lost per electron-ion pair created is defined as

$$
K_{i z} \mathcal{E}_{c}=K_{i z} \mathcal{E}_{i z}+K_{e x} \mathcal{E}_{e x}+K_{e l} \frac{3 m}{M} T_{e}
$$

where $K \equiv\langle\sigma v\rangle$ and $K_{i z}$ and $K_{e x}$ are the electron ionization and excitation collision rate constants, and $\mathcal{E}_{i z}$ and $\mathcal{E}_{e x}$ are the ionization and excitation energy for argon in our case. The third term on the right-hand side of Eq. 6.19 is the electron energy lost in elastic collisions with neutrals. This term can be neglected because of the mass ratio. The total energy lost per ion lost from the system is

$$
\mathcal{E}_{T}=\mathcal{E}_{c}+2 T_{e}+\mathcal{E}_{i}
$$


where $2 T_{e}$ is the mean kinetic energy that an electron carries to the walls, $\mathcal{E}_{i}$ is the sum of the ion energy entering the sheath at the wall and the kinetic energy gained by the ion as it crosses the sheath. The power loss from $\mathcal{E}_{c}$ is power going into plasma production and is given by

$$
P_{\text {production }}=\int_{V} 2 \pi r \mathcal{E}_{c} K_{i z} n_{g} n \mathrm{~d} r \mathrm{~d} z
$$

To estimate the power loss from the wall losses we need the total ion flux to the walls. For a constant $D_{a}$ the diffusion equation

$$
-D_{a} \nabla^{2} n_{i}=\nu_{i z} n_{e}
$$

can be solved and in cylindrical geometry the solution is [22]

$$
n_{i}(r, z)=n_{0} J_{0}\left(\frac{\chi_{01} r}{R}\right) \cos \left(\frac{\pi z}{L}\right)
$$

where $R$ is the plasma radius, $L$ is the plasma length, and $\chi_{01} \approx 2.405$ is the first zero of the Bessel function. As is shown in Section 4.2, the ions are not confined by the dc magnetic field and for a long narrow plasma cylinder the ion flux is almost entirely radial. The radial ion flux is

$$
\Gamma_{i \perp}=-D_{a} \frac{\partial n_{i}}{\partial r} \mid r=R=\frac{\chi_{01} D_{a}}{R} n_{0} J_{1}\left(\chi_{01}\right) \cos \left(\frac{\pi z}{L}\right)
$$

where $D_{a}=\frac{e T_{e}}{M_{i} \omega_{c i}}[1]$. Then the total ion radial flux at the wall is

$$
\int \Gamma_{i \perp}(R, z) 2 \pi R \mathrm{~d} z \approx \frac{5 \times 10^{4} L n_{0} T_{e}}{B}
$$

after substituting for the parameters of our discharge, where $n_{0}$ is the density in the plasma core. The ion velocity entering the sheath is the $\vec{E} \times \vec{B}$ velocity [1] 
given by $u_{\perp} \approx \frac{|e| E_{r}}{M \omega_{c i}}$ where $E_{r} \approx T_{e}|e| R$. The ions are not well confined by the dc magnetic field, and substituting the parameters of our discharge into these estimates we find that $u_{i \perp}$ is almost identical to the Bohm velocity $u_{B}$. The Bohm velocity corresponds to a directed energy of $T_{e} / 2$. The voltage drop in the sheath is approximated by [22]

$$
V_{s}=\frac{T_{e}}{2} \ln \left(\frac{M}{2 \pi m}\right) \approx 4.7 T_{e} \text { for argon }
$$

Therefore, for $\mathcal{E}_{i}$ we obtain $\mathcal{E}_{i} \approx 5.2 T_{e}$. Then the power loss from electron and ion diffusion is

$$
\frac{5 \times 10^{4} L n_{0} T_{e}}{B}\left(2 T_{e}+5.2 T_{e}\right)
$$

and the total power loss from diffusion and plasma production is

$$
\frac{5 \times 10^{4} L n_{0} T_{e}}{B}\left(2 T_{e}+5.2 T_{e}\right)+\int_{V} 2 \pi r \mathcal{E}_{c} K_{i z} n_{g} n \mathrm{~d} r \mathrm{~d} z
$$

which is balanced by the power that the resonant electromagnetic mode deposits into the plasma. Using the functional fit of $K_{i z}$ in [34], we estimate $K_{i z} \approx 6 \times 10^{-15} \mathrm{~m}^{3} / \mathrm{s}$ and solve Eq. 6.28 for $\mathcal{E}_{c}$ for the high-density discharge. The result is that the energy cost per electron-ion pair created is $\mathcal{E}_{c} \approx 25 \mathrm{eV}$. The results are summarized in Table 6.2. We obtain similar results for the low-density discharge.

The calculated value of $\mathcal{E}_{c}$ is comparable with the plot of $\mathcal{E}_{c}$ vs. $T_{e}$ given in $[22]$. 
Table 6.2: Power loss channels in the discharge.

\begin{tabular}{lr}
\hline \hline Total power into plasma & $1040 \mathrm{~W}$ \\
Energy lost per electron-ion pair created $\mathcal{E}_{c}$ & $25 \mathrm{eV}$ \\
Rate of total kinetic energy loss by electron diffusion & $110 \mathrm{~W}$ \\
Rate of total kinetic energy loss by ion diffusion & $286 \mathrm{~W}$ \\
Rate of total energy loss for plasma production & $484 \mathrm{~W}$ \\
\hline \hline
\end{tabular}

\subsection{Stability criterion for a steady-state resonance mode}

The steady-state equilibrium point of the discharge is determined by solving the power balance and particle balance equations in terms of the temperature $T_{e}$. As Eq. 6.25 shows, the total plasma diffusion is given by

$$
\int \Gamma_{i \perp}(R, z) 2 \pi R \mathrm{~d} z=2 \pi \chi_{01} D_{a} n_{0} J_{1}\left(\chi_{01}\right) \int \cos \left(\frac{\pi z}{L}\right) \mathrm{d} z
$$

where $D_{a}=e T_{e} / M_{i} \omega_{c i}[1]$. This is balanced by the total plasma production given by

$$
K_{i z} n_{g} \int_{V} n_{i}(r, z) 2 \pi r \mathrm{~d} r \mathrm{~d} z=2 \pi K_{i z} n_{g} n_{0} \int_{V} J_{0}\left(\frac{\chi_{01} r}{R}\right) \cos \left(\frac{\pi z}{L}\right) r \mathrm{~d} r \mathrm{~d} z
$$

Then particle balance requires that

$2 \pi \chi_{01} D_{a} n_{0} J_{1}\left(\chi_{01}\right) \int \cos \left(\frac{\pi z}{L}\right) \mathrm{d} z=2 \pi K_{i z} n_{g} n_{0} \int_{V} J_{0}\left(\frac{\chi_{01} r}{R}\right) \cos \left(\frac{\pi z}{L}\right) r \mathrm{~d} r \mathrm{~d} z$

which is an equation for $T_{e}$ and where the plasma density $n_{0}$ cancels out. This means that the steady-state temperature calculated from particle balance is independent of the plasma density. Using the particle balance equation and the ionization rate data for argon from [34], we calculated the temperature $T_{e}$ 


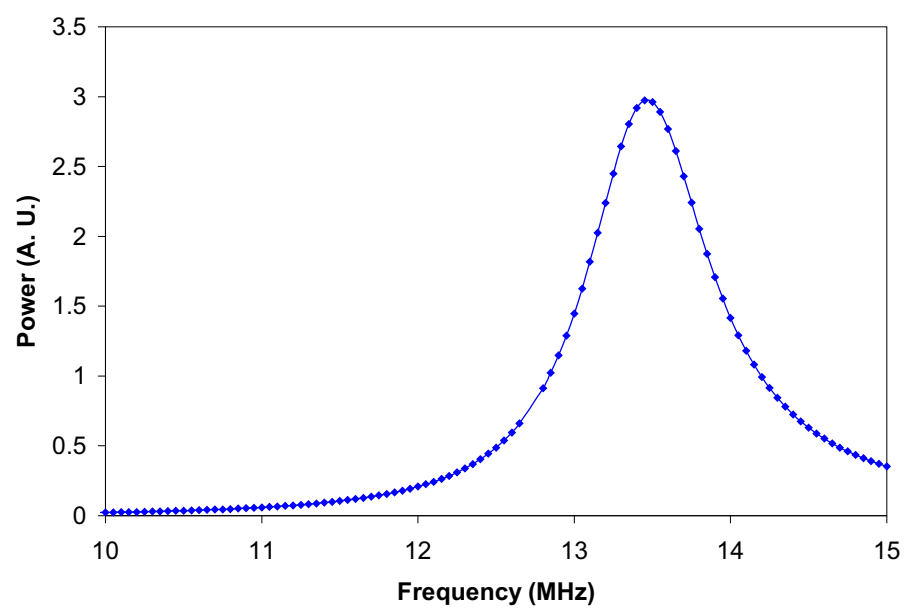

Figure 6.8: Profile of the power absorbed by the plasma as the discharge goes through a resonance.

for the discharge and obtained that $T_{e} \approx 7 \mathrm{eV}$. This theoretical result confirms our experimental estimates obtained with the Langmuir probe of $T_{e} \approx 6.5 \mathrm{eV}$. The power balance is given by

$$
P_{\text {absorbed }}=\left(\mathcal{E}_{i}+\mathcal{E}_{e}\right) \int \Gamma_{i \perp}(R, z) 2 \pi R \mathrm{~d} z+\mathcal{E}_{c} K_{i z} n_{g} \int_{V} n_{i}(r, z) 2 \pi r \mathrm{~d} r \mathrm{~d} z
$$

where $\mathcal{E}_{i}$ is the energy lost per ion lost and $\mathcal{E}_{e}$ is the energy lost per electron lost. In Section 6.6 we estimated $\mathcal{E}_{i} \approx 5.2 T_{e}$ and $\mathcal{E} e \approx 2 T_{e}$. As the discharge goes through the resonance, the profile of the power absorbed by the plasma is given by Fig. 6.8. If we treat the plasma density $n$ as a linear parameter, then as the plasma goes through the resonance, the ionization constant $K_{i z}$ changes nonlinearly in order to keep Eq. 6.32 satisfied. Since $K_{i z}$ is a linear function of $T_{e}$ in the temperature range of the discharge, $T_{e}$ also changes non- 


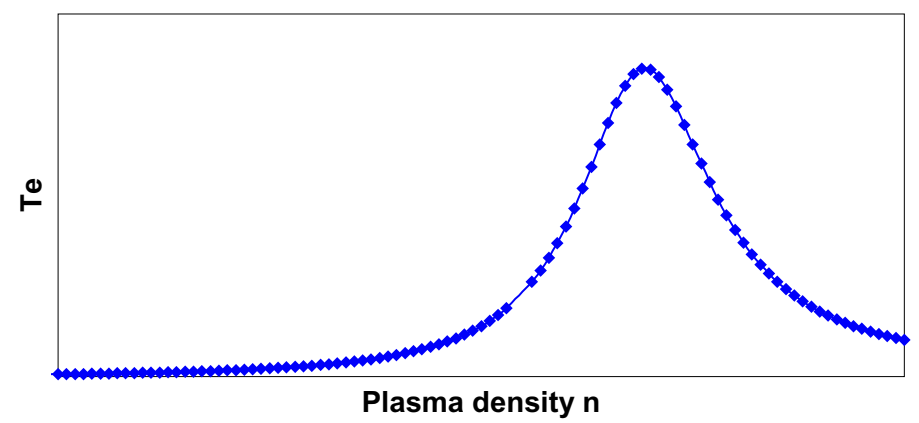

Figure 6.9: Profile of $T_{e}$ obtained from power balance by treating the plasma density $n$ as a linear parameter.

linearly. The temperature profile obtained from power balance is then given by Fig. 6.9. Combining the temperature dependance obtained from power balance and from particle balance, we obtain the stability criterion diagram first calculated by A. Arefiev [1]. The equilibrium points of the discharge are points $\mathrm{A}$ and $\mathrm{B}$ where the particle and power balance have a common temperature. Point $\mathrm{A}$ is unstable because as the density increases (the frequency approaches the resonance frequency), the temperature increases, which increases the ionization rate, thus sustaining the higher density. As the temperature goes through the resonance point, the temperature starts to decrease but the plasma production still exceeds the loss rate and the plasma density continues to increase. The plasma density increases until the discharge reaches point B corresponding to a frequency higher than the resonance frequency. At this point the plasma production is balanced by the loss rate and this is an equilibrium point. This is also the steady-state point, because if the plasma density 


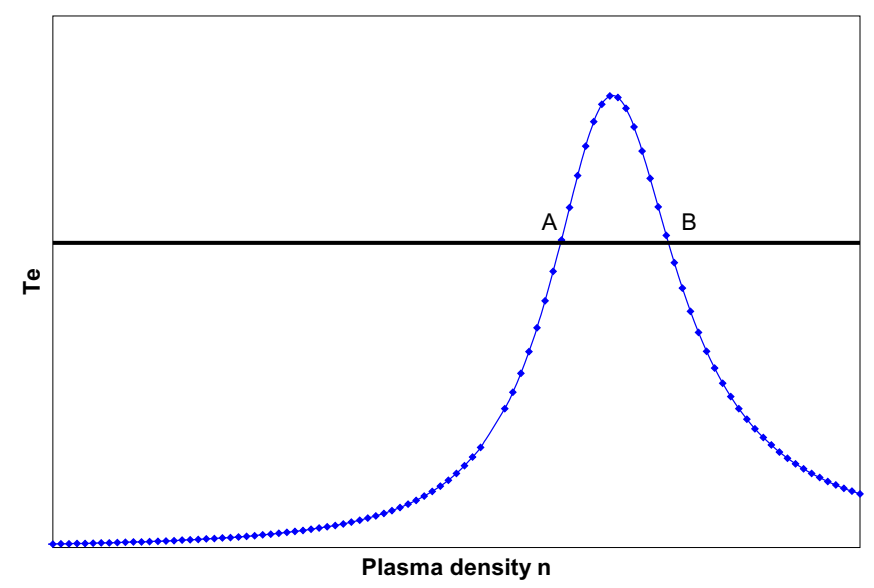

Figure 6.10: The resonance profile of $T_{e}$ is obtained from power balance and the constant profile of $T_{e}$ is obtained from particle balance. The two intersection points are the equilibrium $T_{e}$ of the discharge. Point $\mathrm{A}$ is unstable and point $\mathrm{B}$ is stable.

fluctuates towards a higher value, the temperature drops making the plasma production rate smaller than the diffusion loss rate and the plasma density decreases. If the fluctuation is towards a smaller density, the temperature increases which increases the plasma production rate and the plasma density as well.

If we make the reasonable assumption that the Q-factor of the eigenmode resonance profile does not change as we vary the rf input power into the plasma, Fig. 6.10 shows that the frequency difference between the driving frequency and the eigenmode frequency is proportional to the input rf power. In our experiment that frequency difference is small because the available rf power is relatively low. 


\section{Chapter 7}

\section{Non-Maxwellian electrons}

\subsection{Experimental data}

The presence of fast, non-Maxwellian electrons in many helicon discharges is another aspect of helicon plasmas which is not well understood. Numerous researchers have reported the presence of non-Maxwellian electrons in the helicon discharge $[11,17,18,26]$. Many authors suggest that these electrons are trapped by the axial component of the wave electric field and move with the phase velocity of the helicon wave. On the other hand, the theory of helicon waves based on a uniform plasma model predicts that the axial electric field $E_{\|}$is small. In fact, the collisionless version of that theory predicts $E_{\|}=0$ [13]. A detailed study of non-Maxwellian electrons in helicon plasmas is given by Degeling, Jung, Boswell, and Ellingboe [17]. Their experiment shows that:

- The helicon wave traps electrons from the Maxwellian distribution and accelerates them.

- The large axial electric field $E_{\|}$is obtained only through numerical calculations using the ANTENNA code. 
- The combined effects of the elastic and inelastic collisions scatter the electrons out of the wave frame before they complete a full bounce period thus preventing them from returning energy to the wave.

- The trapped electrons have velocities close to the optimum velocity for ionization $3 \times 10^{8} \mathrm{~cm} / \mathrm{s}$.

- The number of electrons trapped varies as $n_{\text {trapped }} \sim \exp \left(E_{z}^{1 / 2}\right)$.

Far from being an exhaustive study on the subject, we present experimental data for the existence of a non-Maxwellian electron population in our experiment in the context of our discussion of the resonant helicon mode.

As we pointed out in Chapter 4, Section 4.2, the regime in which we operate the Langmuir probe allows us to use Druyvesteyn's formula

$$
\begin{gathered}
g_{e}(V)=\frac{2 m}{e^{2} A}\left(\frac{2 e V}{m}\right)^{1 / 2} \mathrm{~d}^{2} I_{e} / \mathrm{d} V^{2} \\
V=\Phi_{p}-V_{B}>0 \quad \text { (retarding potential region) }
\end{gathered}
$$

where $V$ is the electron energy in volts, $V_{B}$ is the probe voltage, $g_{e}(V)$ is the electron energy distribution function. These formulas apply in the retarding region only, for probe voltages that are negative compared to the plasma potential. The total electron density is determined by

$$
n_{e}=\int_{0}^{\infty} g_{e}(V) \mathrm{d} V
$$

where the integration limits show that to obtain the total electron density we integrate over all probe voltages from $-\infty$ to the plasma potential. To obtain 
the density of a specific electron population we integrate locally over the probe voltage. The average energy of local electron population is determined by

$$
\langle\mathcal{E}\rangle=\frac{1}{n_{e}} \int V g_{e}(V) \mathrm{d} V
$$

We configured our Langmuir probe software to record the I-V trace from every plasma shot and save and average the data. We then use a cubic spline algorithm to fit the I-V trace to a curve and differentiate it. The results are shown in Fig. 7.1, Fig. 7.2, and Fig. 7.3. As we explained in Chapter 4, Section 4.2, we are not operating the probe near the plasma potential because the $\mathrm{I}-\mathrm{V}$ trace is distorted there. The presented data is for sweep voltages that are significantly lower than the estimated plasma potential. The feature that is important in these graphs is the observed localized maximum in $\mathrm{d}^{2} I / \mathrm{d} V^{2}$ due to an increased population of electrons with energies higher than the energy of the bulk electrons. The second derivative also shows a localized minimum which indicates a depletion of the electrons at the energy associated with the minimum. It is reasonable to assume that the mechanism responsible for the creation of the non-Mazwellian population draws electrons from the energy space associated with the minimum of the second derivative and transfers them to the energy space associated with the maximum of the second derivative. The characteristics of the bulk electron population are not reflected in these graph, except that as the I-V trace moves closer to the plasma potential, the magnitude of $\mathrm{d}^{2} I / \mathrm{d} V^{2}$ increases sharply as it approaches the bulk electron population.

The second derivative $\mathrm{d}^{2} I / \mathrm{d} V^{2}$ exhibits some erratic behavior very close to 


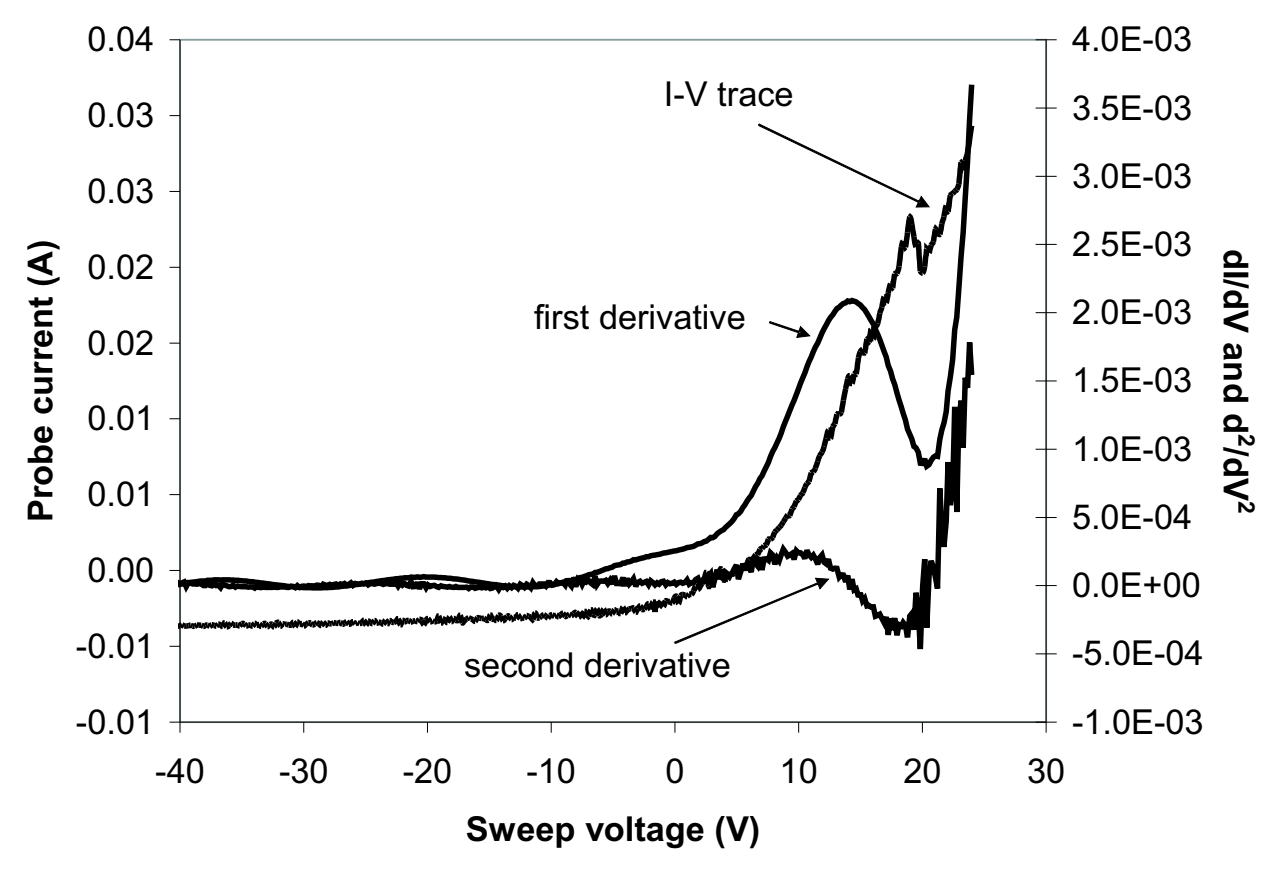

Figure 7.1: High-density discharge, plasma potential estimated at approximately $55 \mathrm{~V}$, radial position of the probe $r \approx 0.0 \mathrm{~cm}$. 


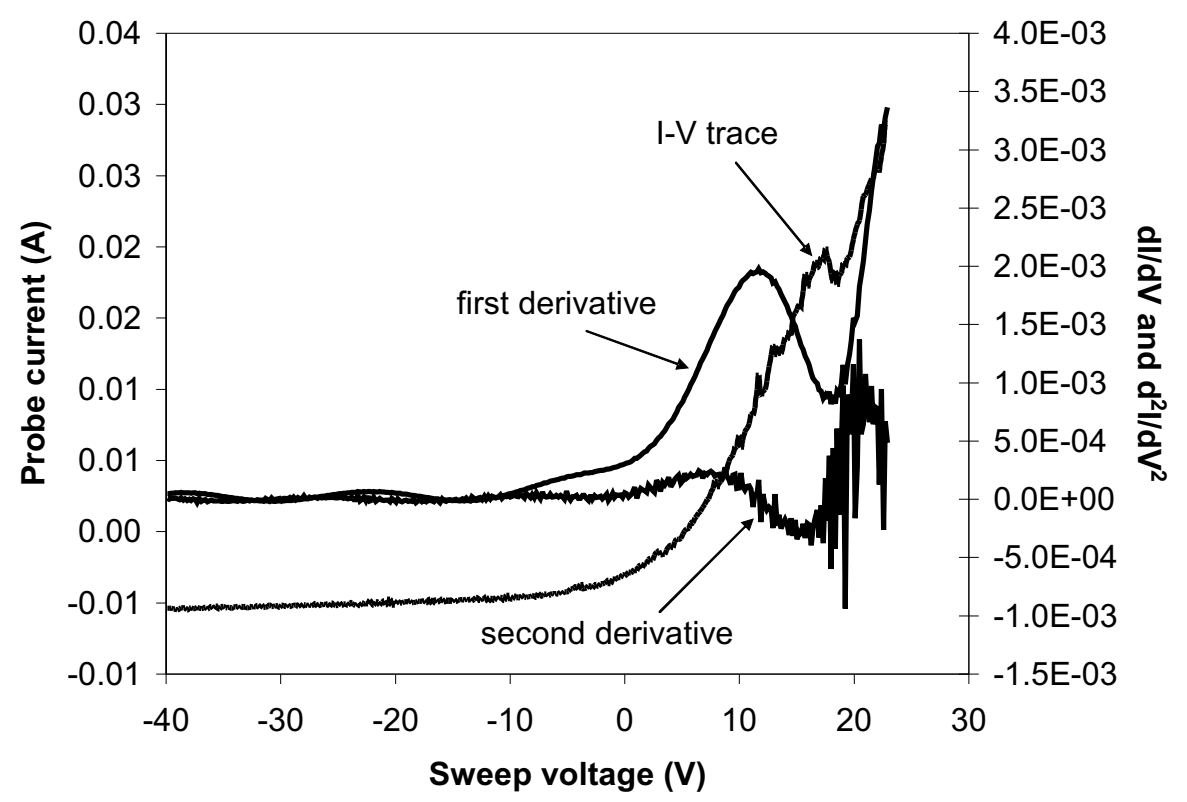

Figure 7.2: High-density discharge, plasma potential estimated at approximately $54 \mathrm{~V}$, radial position of the probe $r \approx 1.0 \mathrm{~cm}$. 


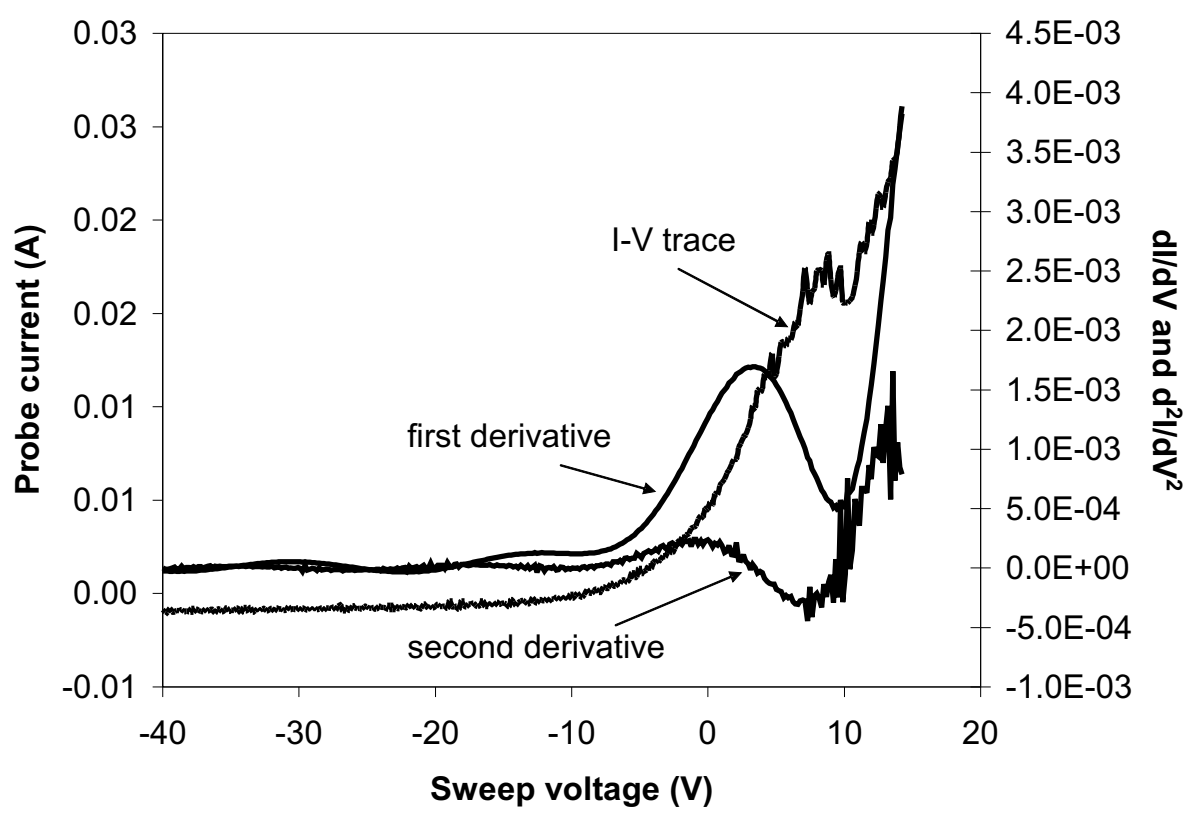

Figure 7.3: High-density discharge, plasma potential estimated at approximately $35 \mathrm{~V}$, radial position of the probe $r \approx 2.0 \mathrm{~cm}$. 
Table 7.1: Characteristics of the population of non-Maxwellian electrons in the high-density discharge.

\begin{tabular}{lccc}
\hline \hline $\mathrm{R}(\mathrm{cm})$ & $\mathrm{n}\left(\mathrm{cm}^{-3}\right)$ & Electron energy $(\mathrm{eV})$ & Plasma potential $(\mathrm{V})$ \\
0.0 & $0.9 \times 10^{10}$ & 23 & 33 \\
1.0 & $1.2 \times 10^{10}$ & 24 & 33 \\
2.0 & $0.6 \times 10^{10}$ & 30 & 30 \\
\hline \hline
\end{tabular}

the maximum value of the sweeping voltage. This is an artifact of the numerical differentiation algorithm and does not reflect any plasma characteristics. Another limitation of the numerical algorithm is the finite number of data points available for differentiation. This limits the resolution of the numerical code.

We use Eq. 7.2 and Eq. 7.3 to integrate around the observed local maximum of $\mathrm{d}^{2} I / \mathrm{d} V^{2}$ and estimate the density and average energy of the observed population of fast electrons. The results for the high-density discharge are summarized in Table 7.1.

We obtain similar results for the low-density discharge.

The data shows that the non-Maxwellian population is relatively uniform in the plasma. In order to estimate the relative importance of the non-Maxwellian electrons, we estimate the relative enhancement of the electron number density at the energy range shown in Table 7.1 over a Maxwellian distribution. The Maxwellian distribution is calculated for the electron temperature of the bulk plasma. Fig. 7.4 compares the observed electron distribution function to a Maxwellian calculated for the temperature of the bulk electrons. The relative increase of non-Maxwellian electrons is estimated as $\mathrm{d} n / n \approx 0.65<1$. This 


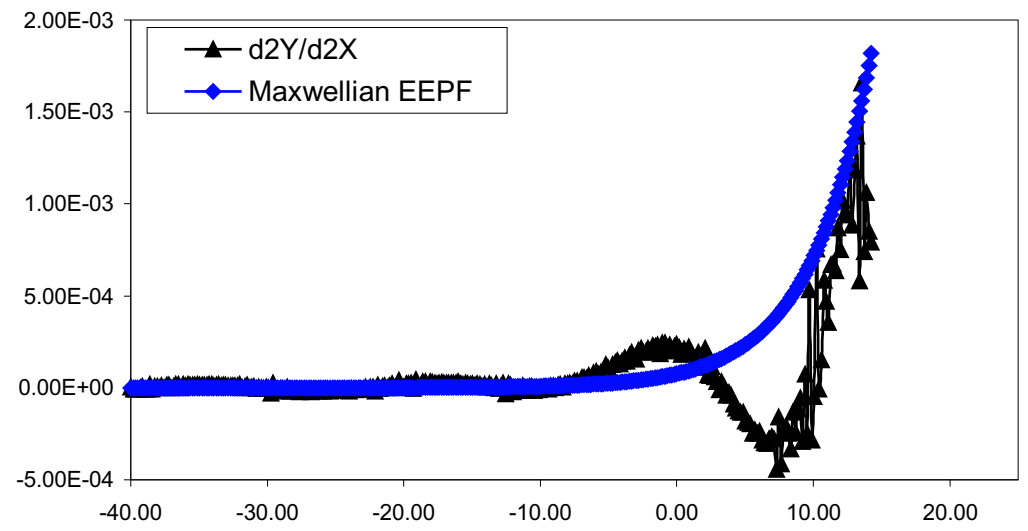

Figure 7.4: Relative enhancement of the electron number density at the observed energy for the non-Maxwellian electrons compared to a Maxwellian distribution.

result suggests that in our discharge the non-Maxwellian electron population plays only a marginal role. However, it is worth showing that the presence and radial distribution of hot electrons in the helicon plasma can be explained by the combined effect of the helicon eigenmode and stochastic heating by the antenna.

\subsection{Physical mechanism}

The data in Table ??, and Table 7.1 reveals that the fast electrons in the helicon plasma are present in the plasma core and in the plasma periphery. The resonant electromagnetic mode discussed in Chapter 5 and Chapter 6 
offers an explanation. Eq. 7.4 shown here

$$
E=E_{\|}\left[1+k_{\|}^{2} r \eta\left(m \frac{\partial g}{\partial r}\right)^{-1}\right]
$$

describes the condition for a singularity in $E_{\|}$which occurs when the term in brackets goes to zero. This electric field traps electrons and creates the hot electron population. The electrons that can be trapped by the wave have velocities determined by

$$
e \Phi_{0} \geq \frac{1}{2} m_{e}\left(v_{z}-v_{\text {phase }}\right)^{2}
$$

where $\Phi_{0}$ is the wave potential well depth, and $v_{z}$ is the range of axial electron velocities that can be trapped. As the plasma approaches resonance conditions, the wave potential well depth $\Phi_{0}$ increases and the wave can trap more electrons from the bulk population according to $n_{\text {trapped }} \sim \exp \left(E_{z}^{1 / 2}\right)$. The bracketed term in Eq. 7.4 simplifies to

$$
1+\frac{r k_{z}^{2} n \omega_{c e}}{\omega \partial n / \partial r}
$$

Using our data for the high-density discharge we can evaluate these quantities and estimate the value of Eq. 7.6. The theoretical results are shown in Fig. 7.5.

Fig. 7.6 shows the experimental values of $\mathrm{d}^{2} I / \mathrm{d} V^{2}$ for the non-Maxwellian peak in the Langmuir I-V trace. The density of these hot electrons is directly proportional to the value of $\mathrm{d}^{2} I / \mathrm{d} V^{2}$. The experimental results show an increase in the non-Maxwellian electron population at $r \approx 0 \mathrm{~cm}, r \approx 1.2 \mathrm{~cm}$, and at the plasma periphery. These results are consistent with the theoretical estimate based on the action of the helicon eigenmode for the case of singularity 


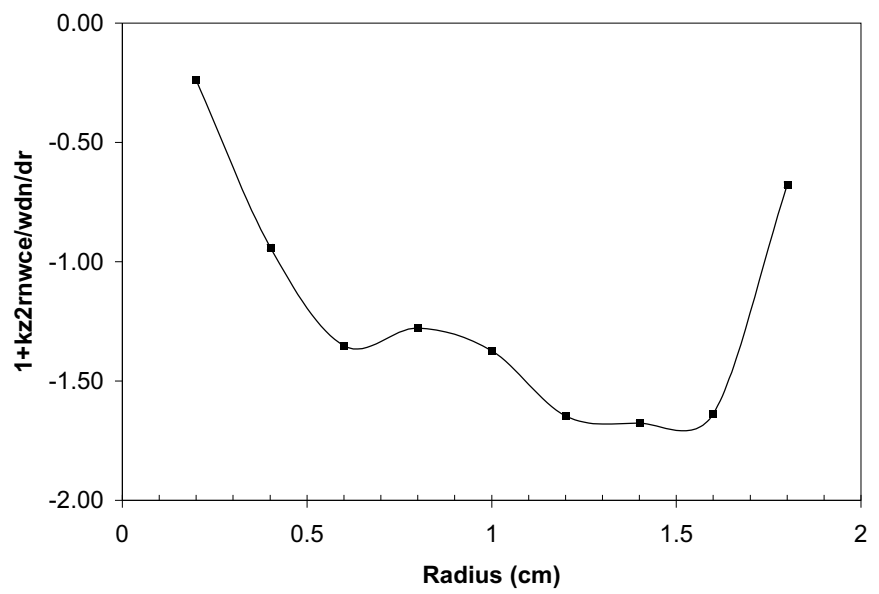

Figure 7.5: Theoretical estimate of the parallel resonance condition for the high-density discharge. Zero crossing would indicate a parallel resonance. The discharge conditions predict near-resonance conditions at $r \approx 0 \mathrm{~cm}$ and at the plasma periphery, plus a spike at $r \approx 1 \mathrm{~cm}$.

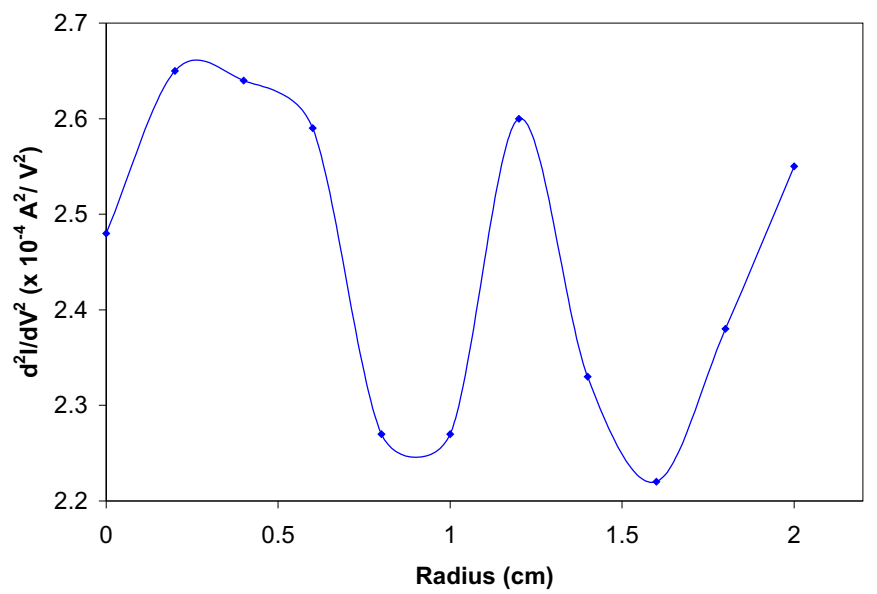

Figure 7.6: Experimental details in the radial profile of the non-Maxwellian electron population for the high-density discharge. The observed spikes in non-Maxwellian electron density are consistent with the theoretical estimate. 


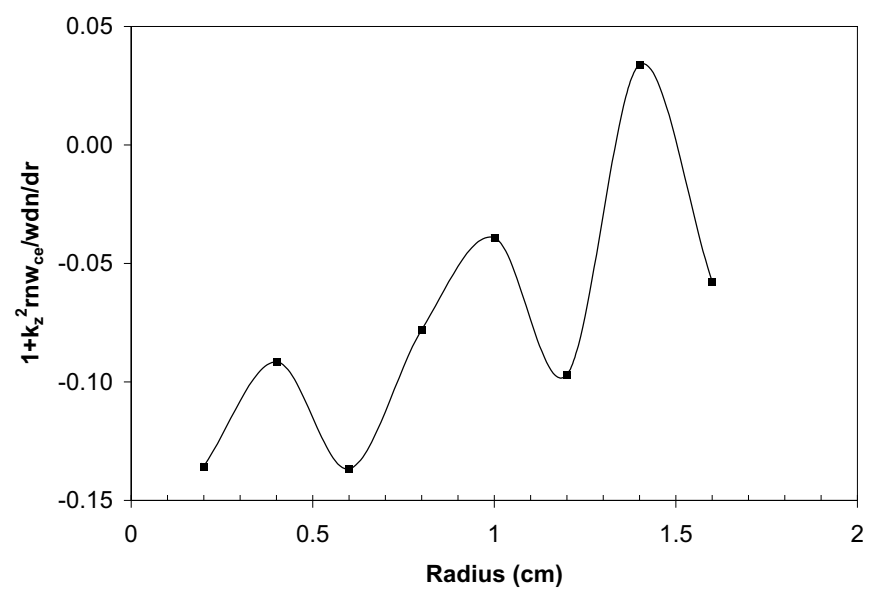

Figure 7.7: Theoretical estimate of the parallel resonance condition for the lowdensity discharge. Zero crossing indicates a parallel resonance. The discharge conditions predict near-resonance conditions at $r \approx 0.4 \mathrm{~cm}, r \approx 1.0 \mathrm{~cm}$, and a resonance at the plasma periphery.

in $E_{\|}$.

Using our data for the low-density discharge we can perform a similar analysis and comparison between the experimental and theoretical estimates for the radial distribution of the hot electrons in the low-density discharge. The results are shown in Fig. 7.7 and Fig. 7.8. The experimental results show an increase in the non-Maxwellian electron population at $r \approx 0.4 \mathrm{~cm}, r \approx 1.0 \mathrm{~cm}$, $r \approx 1.5 \mathrm{~cm}$, and at the plasma periphery. These results are completely consistent with the theoretical estimate for a $E_{\|}$singularity as part of the operation of the helicon resonance eigenmode.

As the plasma density decreases in the outer region of the helicon discharge, the helicon eigenmode goes through a resonance condition on $E_{\|}$, resulting in 


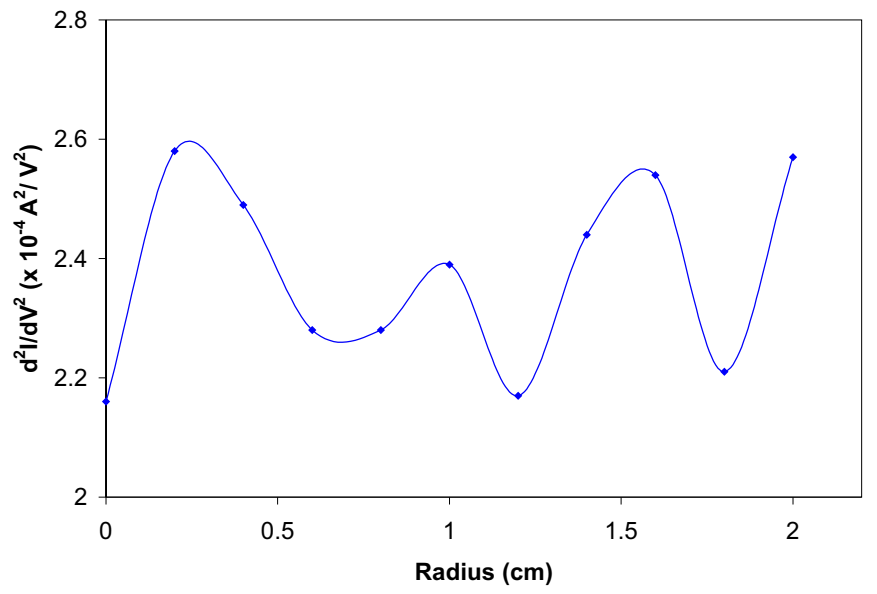

Figure 7.8: Experimental details in the radial profile of the non-Maxwellian electron population for the low-density discharge. The observed spikes in nonMaxwellian electron density are consistent with the theoretical estimate.

an increased non-Maxwellian electron density. We can estimate the energy that the electrons acquire as a result of the proposed mechanism over one $\mathrm{rf}$ period. Fig. 7.9 shows a plot of that estimate. The calculated electron energy is a factor of two smaller than the observed electron energy. However, our estimate is a lower bound which does not include the effect of electron trapping by the resonant parallel electric field.

At the same time, the antenna impedance shown in Section 4.1 suggests that the helical antenna has a strong capacitive component. This creates the possibility that the hot electrons in the outer periphery of the discharge are also the result of a stochastic heating mechanism. In order to investigate this, we added a capacitive antenna to the helicon machine and measured the dependance of the non-Maxwellian population on the operation of the capacitive 


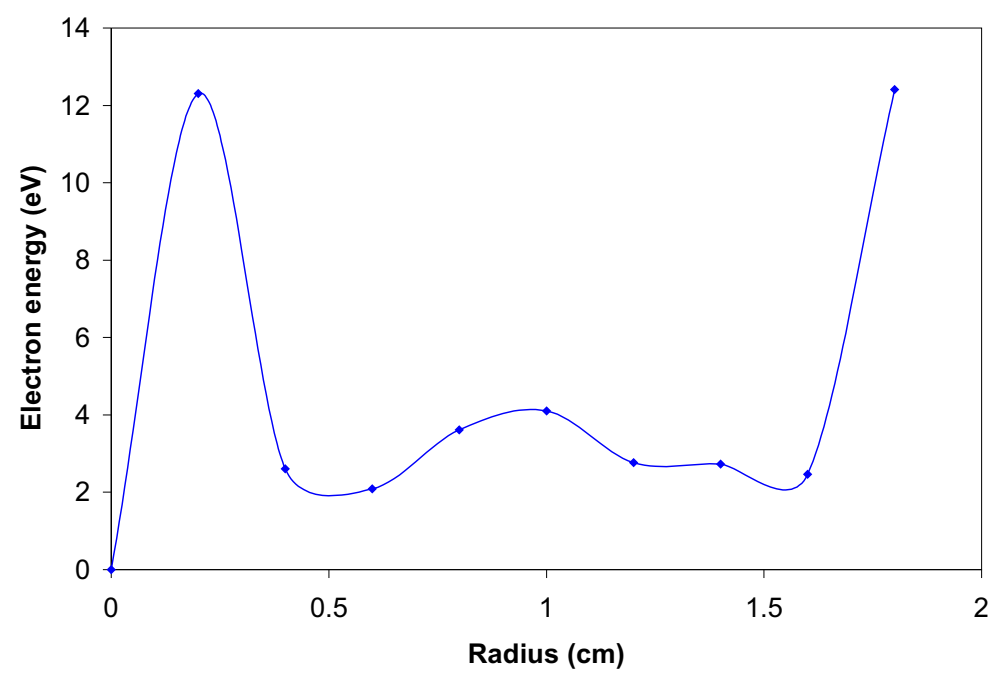

Figure 7.9: Electron energy estimate based on the parallel resonance mechanism over one rf period.

antenna. Since stochastic heating is proportional to the applied antenna voltage, we performed the experiment by varying the input voltage on the capacitive antenna and recorded the signal from the Langmuir probe for various radial positions. The results from the plasma core are shown in Fig. 7.10. The corresponding results from the plasma periphery are shown in Fig. 7.11. The results suggest that at the plasma core the capacitive antenna actually reduces the density of the non-Maxwellian population, while at the plasma periphery the amplitude of the second derivative $\mathrm{d}^{2} I / \mathrm{d} V^{2}$ is proportional to the antenna voltage as expected for stochastic heating. The conclusion is that stochastic heating can play a role in creating a non-Maxwellian electron population at the outer plasma surface where the stochastic heating mechanism is enhanced 


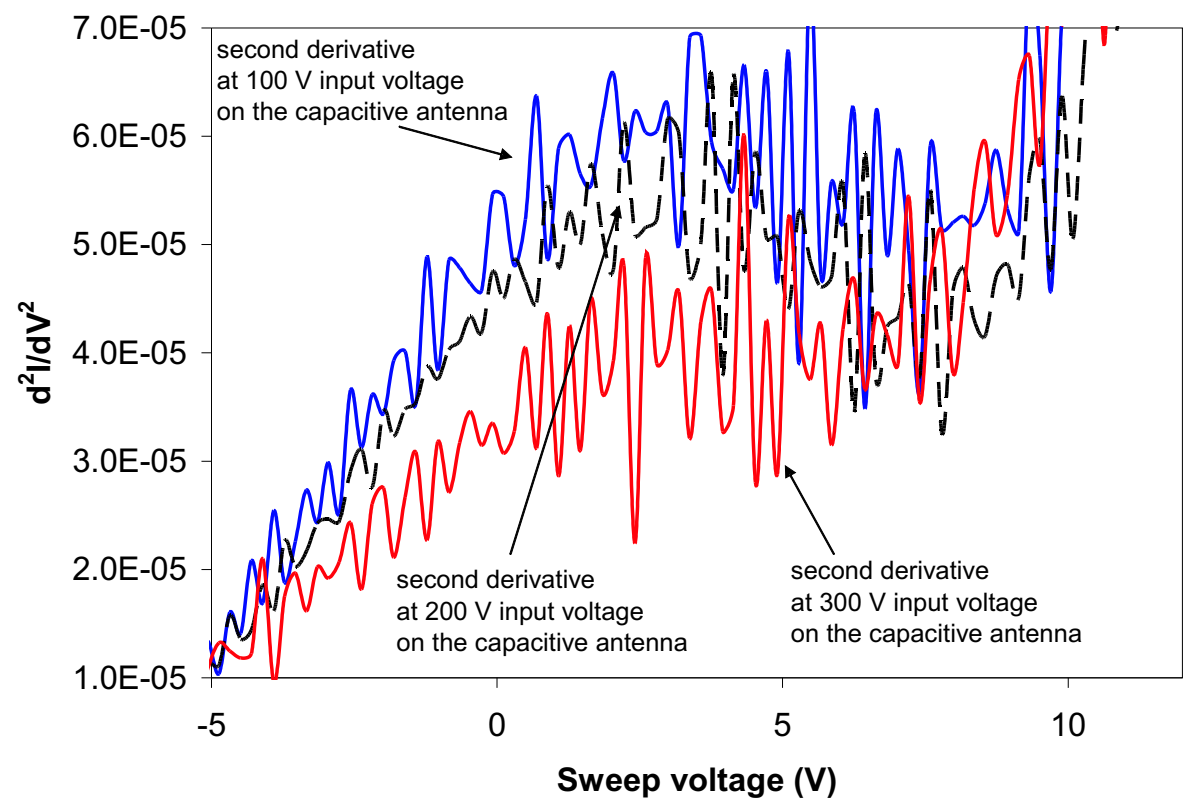

Figure 7.10: Second derivative portion of the $\mathrm{I}-\mathrm{V}$ trace for three different input voltages on the capacitive antenna at $R \approx 0 \mathrm{~cm}$. The graph zooms on the non-Maxwellian portion of the I-V trace. 


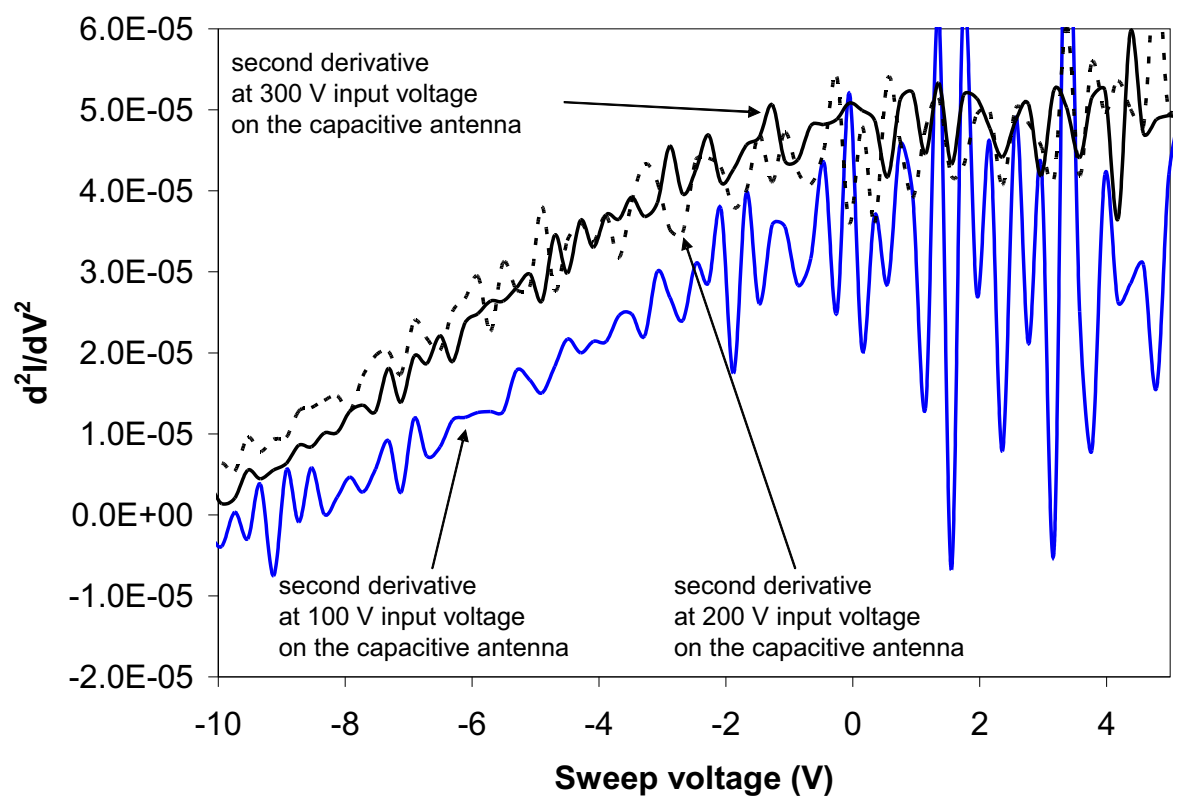

Figure 7.11: Second derivative portion of the I-V trace for three different input voltages on the capacitive antenna at $R \approx 2.0 \mathrm{~cm}$. The graph zooms on the non-Maxwellian portion of the I-V trace. 
by the resonance condition on $E_{\|}$as part of the operation of the helicon eigenmode.

We can evaluate the importance of hot electrons in the discharge by estimating the contribution that the non-Maxwellian electrons make to the overall plasma density in our discharge. In the high-density discharge the hot electrons are about $1 \%$ of the total electron population. Their effective temperature is $16 \mathrm{eV}$ and their $\langle\sigma v\rangle_{i z} \approx 4 \times 10^{-14} \mathrm{~m}^{3} / \mathrm{s}$, while for the bulk electrons $\langle\sigma v\rangle_{i z} \approx 6 \times 10^{-15} \mathrm{~m}^{3} / \mathrm{s}$. This means that a hot electron is 7 times more likely to have a collision resulting in ionization compared to a bulk electron. Therefore, as an estimate we can say that the non-Maxwellian electron population is responsible for about $7 \%$ of the total electron population in our experiment.

\subsection{A practical approach}

Considering a possible practical application for the helicon resonant mechanism, we suggest a way to increase the plasma density by optimizing the operation of the helicon discharge. It is clear from the previous section that the fast electron population in the discharge can be instrumental in increasing the plasma density. In order to increase that population we have to devise a way to maximize the axial electric field $E_{\|}$. A second approach is to optimize the discharge by utilizing the eigenmode frequency.

To optimize the discharge using the eigenmode frequency we suggest that the helicon device be driven by two rf generators. One of them can be 
high-power and with fixed frequency. This rf source will create the helicon discharge and set its parameters, in particular the eigenmode frequency of the plasma. The eigenmode frequency is guaranteed to be lower than the driving frequency. A second, variable frequency can be tuned to the eigenmode frequency and superimposed on the driving frequency. Based on the power level of the variable frequency source, there are two limits of operation: the low limit represents the conditions of our experiment, where the second rf source deposits so little power into the plasma that the discharge is unchanged. The high limit is the case when the second rf source deposits more power into the plasma than the primary generator. In this case the plasma will self-adjust shifting its resonance point to make the second rf frequency the driving frequency. Clearly, between these two extremes the second rf generator can be operated at a power level that optimizes the discharge. The second rf generator tuned to the eigenmode frequency will benefit from a maximum coupling to the plasma because the real component of the plasma impedance is maximized at the eigenmode frequency. This allows for efficient power transfer to the plasma by reducing the amplitude of the rf current at the eigenmode frequency. The decreased rf current reduces the strain on the power delivery system and minimizes the conditions for a breakdown. Most importantly, the reduced current will minimize the ohmic heating in the matching network and antenna which is significant and in our experiment account for about $20 \%$ of the available rf power.

Optimizing the helicon discharge by increasing the non-Maxwellian 
electron population is another approach. Using the variable frequency rf source we can select a frequency (generally different from the eigenmode frequency) that will create a resonance condition on $E_{\|}$in the plasma core. Delivering rf power to the plasma at that frequency will maximize the amplitude of the axial electric field and thus maximize the number of trapped electrons. On the other hand, the driving frequency and antenna geometry can be chosen to result in a phase velocity that produces the optimum electron velocity for ionization as discussed in the previous section.

The two rf generators must drive a single antenna and the two rf frequencies can be mixed with a rf combiner. In the case of optimization using the eigenmode frequency, the two rf signals can deliver power to the antenna through a single matching network because the two frequencies will be close. In the case of optimization using a resonance condition on $E_{\|}$, separate matching networks must be used. The increase in plasma density can be monitored with a rf compensated Langmuir probe, but a more detailed study of the nonMaxwellian electron population will require the construction of a retardingpotential analyzer. 


\section{Chapter 8}

\section{Summary}

This work is part of the ongoing VASIMR project. The aim of our experiment was twofold: to tackle the challenging problem of helicon plasma and to channel our new understanding of the helicon discharge into new ways of improving the operation of the proposed plasma thruster. Along the way we built a fully operational helicon device, complete with diagnostics and datalogging software which the VASIMR group at the Fusion Research Center will use for new and varied plasma research. The key results of this dissertation are summarized below:

- We have presented experimental results which point to the existence of an electromagnetic eigenmode in the helicon plasma.

1. The data suggests that this mode is universal in the helicon discharge and can be excited over a wide range of frequencies.

2. The resonance frequency of the eigenmode is slightly lower than the driving frequency in steady-state as predicted by the discharge stability criterion. 
3. The radial component of the perturbed magnetic field of the eigenmode is peaked at a radius $r^{*}$ where the radial density gradient of the plasma has a maximum.

4. The energy damping rate of the eigenmode is relatively low and cannot deposit the mode energy into the plasma in a single pass.

5. The experimental data also shows that the electromagnetic eigenmode operating at near-resonance is responsible for depositing most of the available rf energy into the plasma.

6. This supports the central conclusion of the theory which predicts that the plasma density gradient forms a potential well which traps the electromagnetic energy. In broader terms, the trapping of the wave in a cavity formed by the density gradients of the plasma both radially and axially provides an explanation to the central dilemma of helicon plasmas: the seeming contradiction between the measured low damping rate of the wave and the measured short damping length.

7. The data suggests that the non-Maxwellian electron population present in most helicon plasmas is created by the electromagnetic eigenmode when the discharge parameters create a resonance condition on $E_{\|}$and we offer an explanation for this aspect of the discharge. Our data also shows the importance of the hot-electron population for the overall particle balance in the discharge. 
- An experimental method is developed and presented to be used as an active probe into the plasma impedance. The method is accurate because it relies on quantities that are measurable in a straightforward and precise way. The presented method also does not require phase measurements which are difficult in the noise-filled environment of a helicon discharge.

- Based on our understanding of the physics of the helicon plasma we offer a practical way of optimizing the operation of the helicon discharge.

The physical framework and experimental results presented in this thesis answer some of the key questions of helicon discharges. Our helicon machine will be used in subsequent experiments to further deepen our understanding of helicon plasmas. A logical next step in the experiment will be to construct miniature magnetic probes that can be used to sample the wave magnetic field under the antenna without significantly perturbing the discharge. Using a fast digitizer, these probes should also be configured to collect the phase information of the resonant helicon mode for various experimental conditions. These measurements will provide important clues to the structure of the wave fields, the spatial extent and characteristics of the potential well created by the density gradient, and the existence of standing waves trapped in the potential well. 


\section{Bibliography}

[1] A. V. Arefiev. Theoretical Studies of the VASIMR Plasma Propulsion Concept. PhD thesis, University of Texas at Austin, 2002.

[2] D. Arnush and F. F. Chen. Generalized theory of helicon waves. ii. excitation and absorption. Physics of Plasmas, 5:1239, 1998.

[3] G. G. Borg and R. W. Boswell. 1998.

[4] A. Boschi and F. Magistrelli. Effect of a rf signal on the characteristic of a langmuir probe. Nuovo Cimento, 29:487, 1963.

[5] R. W. Boswell. Physics Letters, 33A:457, 1970.

[6] R. W. Boswell. Plasma Physics and Controlled Fusion, 26:1147, 1984.

[7] R. W. Boswell. Applied Physics Letters, 47:1095, 1985.

[8] B. N. Breizman and A. V. Arefiev. (private communication, 2003).

[9] B. N. Breizman and A. V. Arefiev. Radially localized helicon modes in nonuniform plasma. Physical Review Letters, 84:3863, 2000.

[10] F. R. Chang-Diaz. The vasimr rocket. Scientific American, Nov. 2000.

[11] F. F. Chen and D. Blackwell. Upper limit to landau damping in helicon discharges. Physical Review Letters, 82:2677, 1999. 
[12] Francis F. Chen. Plasma Physics and Controlled Fusion. Plenum, 1984.

[13] Francis F. Chen. Plasma ionization by helicon waves. Plasma Physics and Controlled Fusion, 33:339, 1991.

[14] Francis F. Chen and D. Arnush. Generalized theory of helicon waves. i.normal modes. Physics of Plasmas, 4:3411, 1997.

[15] Francis F. Chen and D. Arnush. Generalized theory of helicon waves. i.normal modes. Physics of Plasmas, 4:3411, 1997.

[16] J. R. Cogdell. Foundations of Electric Circuits. Prentice, 1999.

[17] A. W. Degeling, C. O. Jung, R. W. Boswell, and A. R. Ellingboe. Plasma production fron helicon waves. Physics of Plasmas, 3:2788, 1996.

[18] A. R. Ellingboe and R. W. Boswell. Electron beam pulses produced by helicon-wave excitation. Physics of Plasmas, 2:1807, 1995.

[19] A. R. Ellingboe and R. W. Boswell. Capacitive, inductive, and heliconwave modes of operation of a helicon plasma source. Physics of Plasmas, 3:2797, 1996.

[20] Chuck Hutchinson, editor. The ARRL Handbook for Radio Amateurs. ARRL, 2001.

[21] P. A. Keiter. Experimental Investigation of Ion Temperature Anisotropy Driven Instabilities in a High Beta Plasma. PhD thesis, West Virginia University, Morgantown, West Virginia, 1999. 
[22] M. A. Lieberman and A. J. Lichtenberg. Principles of Plasma Discharges and Materials Processing. Wiley, 1994.

[23] M. Light and F. F. Chen. Helicon wave excitation with helical antennas. Physics of Plasmas, 2:1084, 1995.

[24] P. Lowenhardt, B. D. Blackwell, R. W. Boswell, G. D. Conway, and S. M. Hamberger. Plasma production in a toroidal heliac by helicon waves. Physical Review Letters, 67:2792, 1991.

[25] P. K. Lowenhardt, B. D. Blackwell, and B. Zhang. A simple miniature magnetic probe with inherent electrostatic rejection. Review of Scientific Instruments, 64:3334, 1993.

[26] A. W. Molvik, A. R. Ellingboe, and T. D. Rognlien. Hot-electron production and wave structure in a helicon plasma source. Physical Review Letters, 79:233, 1997.

[27] Tony Moore, Gil Yetter, Travis Spratlin, and Charlie Nowlin. Radio frequency (rf) measurement and control project report. Technical report, SEMATECH, 1998.

[28] Martin Panevsky and Roger Bengtson. Density gradient dependent helicon modes. In Bulletin of the American Physical Society, volume 47, page 104, 2002.

[29] K. P. Shamrai. Stable modes and abrupt density jumps in a helicon plasma source. Plasma Sources Science and Technology, 7:499, 1998. 
[30] K. P. Shamrai and V. B. Taranov. 1994.

[31] P. C. Stangeby. Effect of bias on trapping probes and bolometers for tokamak edge diagnosis. Journal of Physics D: Applied Physics, 15:1007, 1982.

[32] I. D. Sudit and F. F. Chen. Rf compensated probes for high-density discharges. Plasma Sources Science and Technology, 3:162, 1994.

[33] Isaac D. Sudit and Francis F. Chen. Discharge equilibrium of a helicon plasma. Plasma Sources Science and Technology, 5:43, 1996.

[34] G. S. Voronov. A practical fit formula for ionization rate coefficients of atoms and ions by electron impact: $\mathrm{Z}=1-28$. Atomic Data and Nuclear Data Tables, 65:1, 1997.

[35] P. Zhu and R. W. Boswell. Ar ii laser generated by landau damping of whistler waves at the lower hybrid frequency. Physical Review Letters, $63: 2805,1989$. 


\section{Vita}

Martin Ivanov Panevsky was born in Sofia, Bulgaria on 23 April 1967, the son of Dr.Ivan Panevsky and Margarita Panevsky. He received a Bachelor of Science degree in Physics from Florida Atlantic University in 1997. In the fall of that year Martin started graduate studies at the University of Texas at Austin. In the fall of 1998 Martin joined the VASIMR team at the Fusion Research Center led by Dr.Roger Bengtson and Dr.Boris Breizman.

Permanent address: 2208 Coats Circle Austin, Texas 78748

This dissertation was typeset with $\mathrm{AT}_{\mathrm{E}} \mathrm{X}^{\dagger}$ by the author.

${ }^{\dagger} \mathrm{HT}_{\mathrm{E}} \mathrm{X}$ is a document preparation system developed by Leslie Lamport as a special version of Donald Knuth's T $\mathrm{T}_{\mathrm{E}} \mathrm{X}$ Program. 